\title{
Primeiro tempo de retorno para processos $\beta$-mixing
}

\author{
Erika Alejandra Rada Mora
}

TESE APRESENTADA

AO

Instituto De MATEmÁtica e EstatísticA

DA

Universidade DE SÃo PAUlo

PARA

oBTENÇÃo DO TÍTULO

$\mathrm{DE}$

DOUTOR EM ESTATÍSTICA

Programa: Estatística

Orientador: Prof. Dr. Miguel Natalio Abadi

Durante o desenvolvimento deste trabalho o autor recebeu auxílio financeiro da $\mathrm{CAPES} / \mathrm{CNPq}$

São Paulo, 23 maio de 2014 



\section{Primeiro Tempo de Retorno para Processos $\beta$-Mixing}

Esta versão da tese contém as correções e alterações sugeridas pela Comissão Julgadora durante a defesa da versão original do trabalho, realizada em 23/05/2014. Uma cópia da versão original está disponível no Instituto de Matemática e Estatística da Universidade de São Paulo.

Comissão Julgadora:

- Prof. Dr. Miguel Natalio Abadi (orientador) - IME-USP

- Prof. Dr. Anatoli Iambartsev - IME-USP

- Prof. Dr. Jesús Enrique Garcia - IMECC - UNICAMP

- Prof. Dr. Alexandro Giacomo Grimbert Galo - IM-UFRJ

- Prof. Dr. Yuri Suhov - University of Cambridge 

Dedicado a

Javi 


\section{Agradecimentos}

Ao Professor Miguel Abadi pelo apoio durante o doutorado. Obrigada por me orientar, por suas indicações, por sua paciência infinita e por sua amizade. Sem ele este trabalho não teria sido possível. Aos membros da Comisão Julgadora pelas observações e sugestões feitas que ajudaram a melhorar esta tese.

À Cordinação de Aperfeiçoamento de Pessoal de Nível Superior (CAPES) e ao Conselho Nacional de Desenvolvimento Científico e Tecnológico (CNPq) pelo apoio financeiro durante o desenvolvimento deste trabalho.

A Javi, aunque la palabra gracias se queda bastante corta. GRACIAS por querer compartir parte de su vida conmigo. Por ser mi novio, mi amigo, mi compañero. Por haber hecho un doctorado en paciencia al lado mio. Por su amor y dedicación. Su compañia durante estos años ha sido fundamental. GRACIAS.

A mi familia em Medellín, gracias por haberme apoyado en mis estudios. Gracias por entender mi ausencia y darme fuerzas. A mi papá gracias porque sin él y su esfuerzo no habría llegado tan lejos. Su partida me dió fuerza y espero que en su eterno reposo, sepa que esta tesis es para él. A mi mamá por su apoyo incondicional durante toda mi vida. A mi hermano y amigo Andrés y a mi prima, hermana y amiga Sandra por cubrir mi ausencia. No saben cuanto me han ayudado. A mi hermano Eber por creer en mi. A mis tias Eugenia, Claudia, Lucia e Inés y a mi prima Johana gracias por ese cariño que senti a pesar de los kilometros y a mi mamita Zoraida que rezaba por mi, porque sabía que yo no lo haría. A Nicolás, mi lindo ahijadito, por esperarme y recibirme en cada viaje con ese cariño tan grande.

A mis amigas Sandra, Lina, Oliva, Lenis y Kta, a las que tengo internadas en el alma y a las que simplemente amo, agradezco inmensamente por hacer que esa amistad que tenemos no se muera con la distancia. Me sostuvieron en momentos muy difíciles.

Aos meus queridos amigos em Brasil, Paula, Sandra Zapata, Cristian, Pablo, Naty, Sandro, Rodrigo, Nubia, Deissy, Isa, Jorge, Diane, Ander, Jaime, Enrique, Berna, Sebas, Alex H, Alex V, Susana, Mariana, Thiago, Álvaro, Priscila, John Castillo, Noely e Pedro, além dos 
meus queridos vizinhos Jonatan e Ana, muiiito obrigada pelos momentos compartilhados e pela amizade. Vocês, de um jeito ou de outro, contribuíram no meu doutorado e sem vocês teria sido muito mais complicado.

A meu amigo Elton pela correção do português da tese (os erros que ainda estão são minha culpa). A Jorge, Daniel Azeredo e Kta por me ensinar a simular e a Liliam por me ajudar com uns arquivos de matlab. Obrigradão.

Aos Professores Antonio Galves, Luis Renato Fontes, Fabio Machado e Viviana Giampaoli por sua ajuda burocrática na universidade. Muito obrigada.

Aos funcionarios do Numec e da CPG, obrigada por facilitar com seu trabalho o meu passo pelo instituto.

Existem muitas pessoas que me ajudaram nestes anos de doutorado e que gostaria de agradecer aqui mas acho que falta muito espaço. Muitíssimo obrigada. 


\section{Resumo}

RADA, M. E. A. Primeiro Tempo de Retorno para Processos $\beta$-Mixing. 2014. 83 f. Tese - Instituto de Matemática e Estatística, Universidade de São Paulo, São Paulo, 2014.

Seja $\chi$ um alfabeto finito ou infinito enumerável, e considere $\chi^{n}$ como o conjunto de sequências de tamanho $n$.

No presente trabalho, nós consideramos a função $T_{n}$, definida en $\chi^{n}$ e tomando valores entre 1 e $\infty . T_{n}$ será o primeiro tempo que demora sequência de tamanho $n$, digamos $\omega$, em aparecer de novo sobre uma sequência infinita do processo que começa com $\omega$. Este tempo é conhecido como o tempo de retorno. Seja $S_{n}(\omega)=n-T_{n}(\omega)$ o nosso objeto de estudo, definido também em $\chi^{n}$ e tomando valores entre $-\infty$ e $n-1$.

A função $S_{n}$ foi colocada em evidência, entre outros casos, na análise estatística da Recorrência de Poincaré, e possui relação explícita com a entropia do processo.

Abadi e Lambert, [5] e [19] provaram a convergência da distribuição de $S_{n}$, quando a sequência é escolhida de acordo com a medida produto de $n$ variáveis aleatórias independentes e identicamente distribuídas no alfabeto $\chi$ e como consequência, mostraram a convergência da esperança de $S_{n}$. Nosso trabalho consiste em generalizar o trabalho feito por Abadi e Lambert para processos com uma condição de dependência $\beta$-Mixing.

Palavras-chave: $\beta$-Mixing, Tempo de retorno, Sobreposição, Convergência. 
vi RESUMO 


\section{Abstract}

RADA, M. E. A. First Return Time of the sequence under $\beta$-Mixing conditions.

2014. 83 f. Tese - Instituto de Matemática e Estatística, Universidade de São Paulo, São Paulo, 2014.

We consider the set of finite sequences of length $n$ over a finite or countable alphabet $\chi$. We consider the function defined over $\chi^{n}, S_{n}=n-$ "the first return".

Abadi and Lambert, [5] and [19], computed the exact distribution and the limiting distribution of the $S_{n}$ when the sequence is generated by independent and identically distributed random variables.

Our work consists in a generalization of the work done by Abadi and Lambert in [5] and [19], to processes that verify the $\beta$-Mixing condition and $\left\{X_{n}\right\}_{n \in \mathbb{N}}$ takes values over finite or countable alphabet.

Keywords: $\beta$-Mixing, Return times, Overlapping, Convergence. 


\section{Sumário}

Resumo $\quad$ v

Abstract vii

Lista de Figuras $\quad$ xi

Introdução — xiii

1 Resultados preliminares $\quad 1$

1.1 Notações e definições . . . . . . . . . . . . . . . . . . . . . . . 1

1.1.1 Tempo de Retorno . . . . . . . . . . . . . . . . 3

1.1 .2 Função $S_{n} \ldots \ldots \ldots \ldots \ldots \ldots$. . . . . . . . . . . . . 4

1.1.3 Classificação periódica das palavras . . . . . . . . . . . . . . 5

1.2 Resultados Preliminares . . . . . . . . . . . . . . . . 6

1.2.1 Distribuição limite de $S_{n} \ldots \ldots \ldots \ldots$. . . . . . . . 7

1.2.2 Convergência de $\mathbb{E}\left(S_{n}\right) \ldots \ldots \ldots \ldots$

1.2.3 $\mathbb{E}\left(S_{n}\right)$ e a entropia do processo Bernoulli . . . . . . . . . . . . 9

1.2.4 Não convergência em probabilidade . . . . . . . . . . . . . . . 9

2 Resultados obtidos $\quad 11$

2.1 Distribuição limite de $S_{n} \ldots \ldots \ldots \ldots \ldots \ldots$. . . . . . . . . . . . . . . .

2.1 .1 Exemplos . . . . . . . . . . . . . . . . 26

2.2 Limite da Esperança de $S_{n} \ldots \ldots \ldots \ldots$. . . . . . . . . . . . 32

2.3 Limite do Segundo Momento Finito de $S_{n} \ldots \ldots \ldots$. . . . . . . . . 36

2.4 Resultados condicionais . . . . . . . . . . . . . . . . . . . . 40

2.4 .1 Probabilidade condicional . . . . . . . . . . . . . . . . . . 40

2.4 .2 Esperança condicional . . . . . . . . . . . . . . . . . . . . 44

2.4.3 Esperança da esperança condicional . . . . . . . . . . . . . . 47

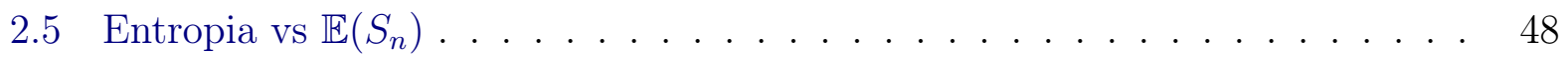

2.6 Caso independente . . . . . . . . . . . . . . . . . . . 51

3 Conclusões e Problemas em aberto $\quad 57$ 
x SUMÁRIO

A Artigo

59

Referências Bibliográficas

61

Índice Remissivo

63 


\section{Lista de Figuras}

1.1 Definição do processo $\beta$-mixing. . . . . . . . . . . . . . . . 2

1.2 Processo com $T_{n}$ maior do que $n \ldots \ldots \ldots \ldots \ldots$

1.3 Relação entre $S_{n}$ e $T_{n}$ com $T_{n}<n . \ldots \ldots \ldots \ldots$

1.4 Relação entre $S_{n}$ e $T_{n}$ com $T_{n} \geq n \ldots \ldots \ldots \ldots$

$1.5 \mathbb{E}\left(S_{n}\right)$ vs. Entropia . . . . . . . . . . . . . . . . . 10

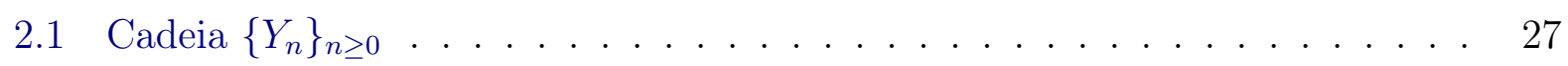

2.2 Convergência 1 de $\mu\left(S_{n}=-1\right)$. . . . . . . . . . . . . . . . . 30

2.3 Convergência 2 de $\mu\left(S_{n}=-1\right)$. . . . . . . . . . . . . . . 31

2.4 Convergência 3 de $\mu\left(S_{n}=-1\right)$. . . . . . . . . . . . . . . 32

$2.5 E\left(S_{n}\right)$ para o processo de Markov com gramática completa. . . . . . . . . 49

2.6 Entropia para o processo de Markov com gramática completa. . . . . . . . . 49

$2.7 E\left(S_{n}\right)$ para o processo de Markov com gramática completa . . . . . . . . . 50

2.8 Entropia para o processo de Markov com gramática completa . . . . . . . . 51

2.9 Limitantes superior e inferior para o limite de $E\left(S_{n}\right) \ldots \ldots$. . . . . . . . 52

2.10 Limitantes superior e inferior para o limite do segundo momento de $E\left(S_{n}\right)$. . 54

2.11 Limitantes superior e inferior para o limite da Variância de $E\left(S_{n}\right) \ldots$. . . . . 56 


\section{Introdução}

Considere um processo estocástico definido sobre o conjunto $\chi$, finito ou infinito enumerável. Nós nos referiremos a este conjunto como o alfabeto. Para cada entero positivo $n$, considere o conjunto de todas sequências de tamanho $n$ formada com elementos de este alfabeto, esto é $\chi^{n}$. A estas sequências daremos o nome de palavras e denotaremos elas com a letra $\omega$ (ver $1.1)$.

No presente trabalho, nós consideramos a função $S_{n}$, definida sobre $\chi^{n}$ e tomando valores entre $-\infty$ e $n-1$. Para cada palavra de tamanho $n$, essa função assume o valor do tamanho da palavra menos o primeiro tempo de retorno dela, ou seja, $S_{n}=n-$ primeiro tempo de retorno. O primeiro tempo de retorno de una palavra $\omega$ de tamanho $n$ é o tempo que demora a palavra em aparecer de novo sobre uma sequência infinita do processo que começa com $\omega$. Vamos denotar $T_{n}$ este tempo de retorno. Se $S_{n}$ for maior que zero, a palavra apresenta uma sobreposição com uma cópia dela mesma transladada, [19]. Se, pelo contrário $S_{n}$ é negativo, o valor absoluto da função representa o tempo entre a palavra e sua nova aparição.

A relevância da função $T_{n}$ (e portanto de $S_{n}$ ) foi colocada em evidência na análise estatística da Recorrência de Poincaré. Para provar a convergência do número de ocorrência de palavras (digamos, de tamanho $n$ ) quando $n$ diverge, para a distribuição de Poisson, é necessário que a palavra não se sobreponha com ela mesma [17]. Ou, pelo menos, que a proporção das palavras que se sobrepõem seja pequena com relação a $n$ [7]. Se esse não for o caso, a distribuição limite tem lei Poisson composta [16]. Também temos na literatura algumas aproximaçoẽs para esse limite em [20], [21] e [22].

Tal relevância também aparece quando consideramos o tempo decorrido até a primeira ocorrência da palavra. Esse tempo é conhecido como o Tempo de Ocorrência ou Hitting time (ver 1.8). É sabido que o tempo de ocorrência pode ser aproximado por uma lei exponencial com parâmetro dado pela medida da palavra, no caso em que a palavra possui uma sobreposição com ela mesma ou quando tal sobreposição é "pequena". Mas, quando a palavra apresenta sobreposição (que não seja "pequena") com ela mesma, o parametro deve ser corrigido por um fator. Tal fator é a probabilidade da palavra não aparecer duas vezes consecutivas. E essa probabilidade é dada pelas propriedades de sobreposição da palavra, [1] e [3]. 
Uma situação similar acontece quando consideramos o tempo decorrido até a primeira ocorrência da palavra, colocando como condição inicial a ocorrência da própria palavra. Este tempo é chamado de Tempo de Retorno (Return Time). Neste caso, acontece o mesmo. É sabido que o tempo de retorno também pode ser bem aproximado por uma lei exponencial. Mas quando a palavra apresenta sobreposição com ela mesma, essa lei deixa de ser exponencial, passando a ser uma combinação convexa de uma medida degenerada na origem e uma exponencial [13]. Neste caso, o mesmo fator citado no paraǵrafo anterior, aparece na combinação convexa e a lei exponencial.

Até onde sabemos, os primeiros a observarem que a medida de todas as palavras que possuem "grandes" sobreposições converge para zero foram Collet, Galves \& Schmitt, em 1999 [13]. Nesse trabalho, os autores provaram o decaimento exponencial dessa medida quando "grande" significa maior ou igual a $n-n / 3$. Tal resultado vale para processos misturadores com função $\psi$ de decaimento exponencial. Mais tarde, o mesmo resultado foi generalizado em [1] para processos misturadores com função $\phi$ de decaimento exponencial. No último caso, "grande" significa ser maior ou igual a $n-c n$, onde $c$ é uma constante que depende da cardinalidade do alfabeto.

A distribuição de $T_{n}$ é desconhecida. Ou seja, não há uma forma explícita para

$$
F_{T_{n}}:=\mu\left(T_{n} \leq y\right) .
$$

Em 2002 usando a complexidade de Kolmogorov, Saussol, Troubetzkoy \& Vaienti [25] provaram, para um processo ergódico com entropia positiva, cumprindo propriedade de especificação [9], que

$$
\lim _{n \rightarrow \infty} \frac{T_{n}}{n}=1 \text { q.c }
$$

Esta expressão diz que, assintóticamente, $T_{n}$ cresce na mesma velocidade que $n$. Isto sugere que a variável aleatória $T_{n}$ apresenta um comportamento assintótico defectivo. Ou seja, temos então uma sequência de variáveis aleatórias não estocasticamente limitadas. Aí vemos a necessidade de definir convenientemente uma outra sequência de variáveis aleatórias que nos forneça informação sobre o Primeiro Tempo de Retorno. Neste trabalho, tal função será $S_{n}$.

Em 2003, usando o Teorema de Shannon, McMillan \& Breiman, Afraimovich, Chazottes e Saussol provaram que vale o mesmo resultado que se apresenta em 1, [9]. Este resultado também foi provado para uma clase de funções não uniformemente expandidas do intervalo [18] no contexto de sistemas dinâmicos.

O principio de grandes desvios para $T_{n}$ tem sido e continua sendo motivo de estudo. Abadi e Vaienti [7] encontraram a função de grandes desvios e relacionaram $T_{n}$ com a entropia de Renyi do processo gerador de palavras. Abadi e Cardeño, [4] estudaram a entropia de Renyi 
e a função de grandes desvios de $T_{n}$, para processos com condições de mistura. Haydn e Vaienti [15] estudaram o mesmo principio e sua relação com a entropia de Renyi, mas assumindo condições de decaimento exponencial. Logo o limite da função de grandes desvios esta relacionado com a entropia do processo. Então é natural perguntar pelas flutuações de $T_{n}$. Ou seja, perguntar pela existência da distribuição limite re-escalada de $T_{n}$,

$$
\frac{T_{n}-\mathbb{E}\left(T_{n}\right)}{\sqrt{\left(\operatorname{Var}\left(T_{n}\right)\right)}},
$$

incluindo o problema do comportamento asintótico de $\mathbb{E}\left(T_{n}\right)$ e de $\operatorname{Var}\left(T_{n}\right)$.

Motivados pelo estudo da distribuição limite de $S_{n}$ como uma fonte de informação para encontrar propriedades interessantes anunciadas anteriormente de $T_{n}$, Lambert [19] Abadi \& Lambert [5] encontraram a convêrgencia da distribuição de $S_{n}$ para uma distribuição limite cuja cauda decai exponencialmente, quando $n$ diverge. Esto foi provado considerando um processo independente e identicamente distribuído.

Além disso, eles apresentaram um limite superior para a velocidade dessa convergência. Tal limite superior é não-uniforme, porêm permite obter como corolário a esperança e em geral, todos os momentos finitos de ordem polinomial.

O objetivo deste trabalho é generalizar estos resultados para processos com dependência do tipo $\beta$-mixing para gerar as palavras (ver 1.2). Dessa maneira, definimos a função $S_{n}$ e apresentamos o limite para $\mu\left(S_{n} \geq k\right)$, com $k$ inteiro não negativo, que quando o processo tem gramática completa (ou seja, todas as possíveis combinações de símbolos do alfabeto têm probabilidade positiva de ocorrência, ver 1.6) será a distribuição limite de $S_{n}$. Além disso, como consequência do anterior, apresentamos o limite para o valor esperado, o segundo momento finito e a variância de $S_{n}$ com suas respectivas velocidades de convergência.

Estudamos também o caso independente trabalhado em [19] um pouco mais e apresentamos limitantes mais eficientes para o limite da esperança de $S_{n}$, o qual nos permitiu calcular limitantes para o segundo momento finito e consequentemente, para a variância. 


\section{Capítulo 1}

\section{Resultados preliminares}

\subsection{Notações e definições}

Neste trabalho vamos considerar processos estocásticos estacionários sobre um conjunto $\chi$ finito ou infinito enumerável. Tal conjunto será chamado de alfabeto. Aqui consideraremos sempre um alfabeto $\chi$ tal que o cardinal dele, $|\chi|$, seja maior o igual a 2 .

Definição 1.1. Diremos que uma palavra é uma sequência finita de símbolos do alfabeto

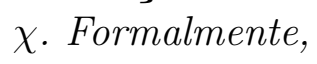

$$
x_{1}^{n}=\left(x_{1} x_{2} \cdots x_{n}\right) x_{i} \in \chi, i=1,2, \ldots, n .
$$

onde os indices indicam onde começa e onde termina a palavra.

O conjunto de todas as palavras de tamanho $n$ com símbolos no alfabeto $\chi$ será denotado por $\chi^{n}$.

Vamos denotar uma palavra $x_{1}^{n}$ também como $\omega$ ou $\omega_{n}$ se quisermos especificar a palavra com seu tamanho.

Consideraremos um processo estocástico para gerar as palavras com uma dependência tipo $\beta$-mixing, cuja definição, extraída de [11], será dada a seguir.

Seja $\left\{X_{n}\right\}_{n \in \mathbb{Z}}$ um processo estocástico estacionário assumindo valores no alfabeto $\chi$. O valor de $X_{n}$ é interpretado como o estado do processo no tempo $n$. Para $-\infty \leq J \leq L \leq \infty$, definimos a $\sigma$-álgebra

$$
\mathcal{F}_{J}^{L}:=\sigma\left(X_{k}, J \leq k \leq L(k \in \mathbb{Z})\right) .
$$

Ou seja, $\mathcal{F}_{J}^{L}$ é a $\sigma$-álgebra gerada pelas variáveis aleatórias $X_{k}$ para $k$ entre $J$ e $L$.

Definição 1.2. Para quaisquer duas $\sigma$-álgebras $\mathcal{F}_{J}^{L}$ e $\mathcal{F}_{M}^{N}$, definimos a medida de dependência $\beta$-mixing como

$$
\beta\left(\mathcal{F}_{J}^{L}, \mathcal{F}_{M}^{N}\right):=\sup \frac{1}{2} \sum_{i=1}^{I} \sum_{k=1}^{K}\left|\mu\left(A_{i} \cap B_{k}\right)-\mu P\left(A_{i}\right) \mu\left(B_{k}\right)\right|,
$$

onde o supremo é tomado sobre todos os pares de partições finitas $\left\{A_{1}, \ldots, A_{I}\right\}$ e $\left\{B_{1}, \ldots, B_{K}\right\}$ de $\chi^{\mathbb{Z}}$ tais que $A_{i} \in \mathcal{F}_{J}^{L}$ para cada $i$ e $B_{k} \in \mathcal{F}_{M}^{N}$ para cada $k$.

Definição 1.3. Para $n \geq 1$, definimos o coeficiente $\beta$-mixing por,

$$
\beta(n):=\sup _{j \in \mathbb{Z}} \beta\left(\mathcal{F}_{-\infty}^{j}, \mathcal{F}_{j+n}^{\infty}\right)
$$


Na seguinte figura se mostra o conjunto $A \in \mathcal{F}_{-\infty}^{j}$ e o conjunto $B \in \mathcal{F}_{j+n}^{\infty}$. O coeficiente $\beta$ destes dois conjuntos está baseado na distância que existe entre eles.

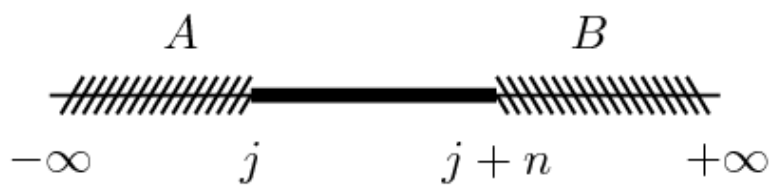

Figura 1.1: Definição do processo $\beta$-mixing.

Definição 1.4. Diremos que o processo $\left\{X_{n}\right\}_{n \in \mathbb{Z}}$ é $\beta$-mixing se

$$
\lim _{n \rightarrow \infty} \beta(n)=0 .
$$

Uma característica da função $\beta$ é que ela é não-crescente ou decrescente. Para provar isto, basta aplicar a definição observando que $\mathcal{F}_{j+n+1}^{\infty} \subseteq \mathcal{F}_{j+n}^{\infty}$.

A ideia por trás de um processo com dependência tipo mixing é garantir que a probabilidade de ocorrência simultânea de dois eventos converge ao produto das probabilidades, quando o tempo de ocorrência entre os eventos tende para infinito. Ou seja, a dependência tipo $\beta$-mixing garante uma perda de memória do processo.

O coeficiente $\beta$-mixing foi introduzido por Kolmogorov e apareceu por primeira vez no artigo escrito por Wolkonski \& Rozanov em 1959 [23]. Como exemplo de um processo $\beta$ mixing podemos apresentar as cadeias de Markov estritamente estacionárias, irredutíveis e aperiódicas [11].

Outra medida de dependência tipo mixing para processos estocásticos é a dependencia $\alpha$ mixing. Nós será útil na prova de nosso teorema principal, porque se um processo estocástico é $\beta$-mixing, então também é $\alpha$-mixing [11].

Definição 1.5. Para quaisquer duas $\sigma$-álgebras $\mathcal{F}_{J}^{L}$ e $\mathcal{F}_{M}^{N}$, definimos a medida de dependência $\alpha$-mixing como

$$
\alpha\left(\mathcal{F}_{J}^{L}, \mathcal{F}_{M}^{N}\right):=\sup |\mu(A \cap B)-\mu(A) \mu(B)|, \quad A \in \mathcal{F}_{J}^{L}, \quad B \in \mathcal{F}_{M}^{N} .
$$

Para $n \geq 1$, definimos o coeficiente $\alpha$-mixing por,

$$
\alpha(n):=\sup _{j \in \mathbb{Z}} \alpha\left(\mathcal{F}_{-\infty}^{j}, \mathcal{F}_{j+n}^{\infty}\right)
$$

Finalmente, diremos que o processo $\left\{X_{n}\right\}_{n \in \mathbb{Z}}$ é $\alpha$-mixing se

$$
\lim _{n \rightarrow \infty} \alpha(n)=0 .
$$

Uma definição muito útil, é a definição de um processo com gramática completa, a qual será dada a seguir. 
Definição 1.6. Sejam $\left\{X_{n}\right\}_{n \in \mathbb{N}}$ um processo estocástico definido sobre o alfabeto $\chi$ e $\mu$ uma medida de probabilidade do processo em $\chi^{\mathbb{N}}$. Diremos que o processo tem gramática completa se para todo $x_{1}^{n} \in \chi^{n}, \mu\left(x_{1}^{n}\right)>0$.

\subsubsection{Tempo de Retorno}

Agora, trataremos do tempo de retorno, que é uma definição essencial no presente trabalho. Para isso, definimos o tempo de ocorrência. Em palavras: dizemos que o tempo de ocorrência de uma palavra é o mínimo tempo que leva a palavra em aparecer numa sequência infinita dada.

Definição 1.7. Para uma palavra $x_{1}^{n} \in \chi^{n}$ dada, o tempo de ocorrência de $x_{1}^{n}$ em $y_{0}^{\infty}$, denotado por $\tau_{x_{1}^{n}}$, é dado por

$$
\tau_{x_{1}^{n}}\left(y_{0}^{\infty}\right)=\inf \left\{t \geq 1 \mid y_{t}^{t+n-1}=x_{1}^{n}\right\}
$$

e infinito se o conjunto for vazio.

Motivados pelos trabalhos [1], [2], [6] e [14], nós estudamos o primeiro tempo de retorno de uma sequência de tamanho $n$.

Definição 1.8. O primeiro tempo de retorno de uma sequência $x_{1}^{n}$ é dado pelo infimo dos tempos de ocorrência $\tau_{x_{1}^{n}}\left(y_{0}^{\infty}\right)$ sobre todas as realizações possíveis $y_{0}^{\infty}$ que começam com $x_{1}^{n}$. Isto é,

$$
T_{n}\left(x_{1}^{n}\right)=\inf _{y_{0}^{\infty}: y_{0}^{n-1}=x_{1}^{n}} \tau_{x_{1}^{n}}\left(y_{0}^{\infty}\right)
$$

Note que o tempo de retorno é uma variável aleatória. Este tempo tem uma versão usada em Sistemas Dinâmicos. Considere $x_{1}^{\infty} \in \chi^{\mathbb{N}}$. Defina agora uma translação $\sigma$, transformação shift, da seguinte maneira:

$$
\begin{aligned}
& \sigma: \chi^{\mathbb{N}} \rightarrow \chi^{\mathbb{N}}, \\
& \sigma\left(x_{1}^{\infty}\right)=x_{2}^{\infty},
\end{aligned}
$$

onde $\sigma$ retira da sequência o primeiro símbolo. Aplicando recursivamente $\sigma$, temos que para $1 \leq m$ :

$$
\sigma^{m}\left(x_{1}^{\infty}\right)=x_{m+1}^{\infty}
$$

Então, finalmente, definimos $\tilde{T}_{n}(\omega)$ da seguinte maneira:

Definição 1.9 .

$$
\tilde{T}_{n}(\omega)=\inf _{y \in \chi^{\mathbb{N}}: y_{0}^{n-1}=x_{1}^{n}}\left\{m \geq 1: \sigma^{m}(y)=y\right\}
$$

onde o infimo acima é tomado entre todas as sequências y de tamanho infinito que tenham os mesmos $n$ primeiros símbolos de $\omega$.

A diferença entre $\tilde{T}_{n}$ e $T_{n}$ está em que $\tilde{T}_{n}$ está definido sobre $\chi^{\mathbb{N}}$ e $T_{n}$ sobre $\chi^{n}$ mas se o processo que gera as palavras tem grámatica completa, $y$ sempre pode ser tomado de maneira que $1 \leq \tilde{T}_{n}(y) \leq n$, nessas condições, $\tilde{T}_{n}$ e $T_{n}$ são equivalentes.

Exemplo 1.10. Seja $x_{1}^{6}=010101$ uma palavra de tamanho 6. O infimo de todas as possíveis sequências o garante $y_{0}^{\infty}=0101010101 \ldots$ se este elemento pertence ao espaço amostral. Logo, 
se nós transladássemos a palavra dois passos à direita sobre $y_{0}^{\infty}$, encontraremos novamente $x_{1}^{6}$. A seguinte figura representa gráficamente, as translaçoões dadas.

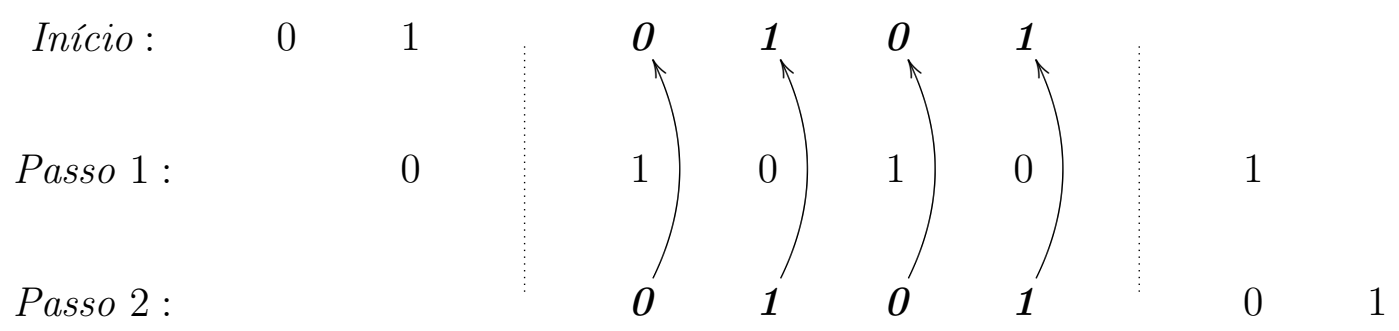

Como esta palavra retorna na segunda translação, o tempo de retorno $T_{6}(010101)=2$.

No seguinte exemplo mostramos que o tempo de retorno de uma palavra pode ser maior do que o tamanho da palavra.

Exemplo 1.11. Considere uma cadeia de Markov em $\chi=\{0,1,2\}$ tal que

$$
\begin{aligned}
& \begin{array}{lll}
0 & 1 & 2
\end{array} \\
& P=\begin{array}{l}
0 \\
1 \\
2
\end{array}\left(\begin{array}{ccc}
1-q_{0} & q_{0} & 0 \\
1-q_{1} & 0 & q_{1} \\
1-q_{2} & 0 & q_{2}
\end{array}\right)
\end{aligned}
$$

onde $0<q_{i}<1$, para $i=0,1,2$.

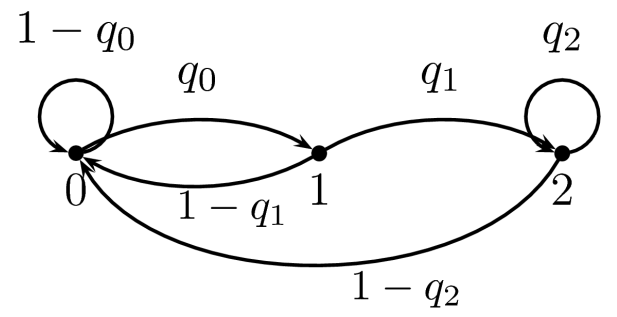

Figura 1.2: Processo com $T_{n}$ maior do que $n$.

Seja $x_{1}^{4}=1012$. Note que nenhuma palavra $y_{0}^{\infty}$ que comece com 1012 vai repeti-la imediatamente pois $p_{2,1}=0$. Logo, $T_{4}(1012)>4$.

\subsubsection{Função $S_{n}$}

Na presente seção, iremos construir uma nova variável aleatória relacionada à variável $T_{n}$, a qual é a estrela deste trabalho. Para palavras geradas por sequências de variáveis aleatórias independentes com marginais identicamente distribuídas, se provou em [19] e [5] que essa variável aleatória possui distribuição limite. Este resultado motivou esta tese e foi generalizado no capítulo 2.

Para começar, construimos a função $S_{n}: \chi^{n} \rightarrow \mathbb{Z}$ por médio da seguinte equação:

$$
S_{n}\left(x_{1}^{n}\right)=n-T_{n}\left(x_{1}^{n}\right)
$$

A figura seguinte mostra graficamente a relação entre $S_{n}$ e $T_{n}$, sendo $\omega$ uma palavra infinita. 


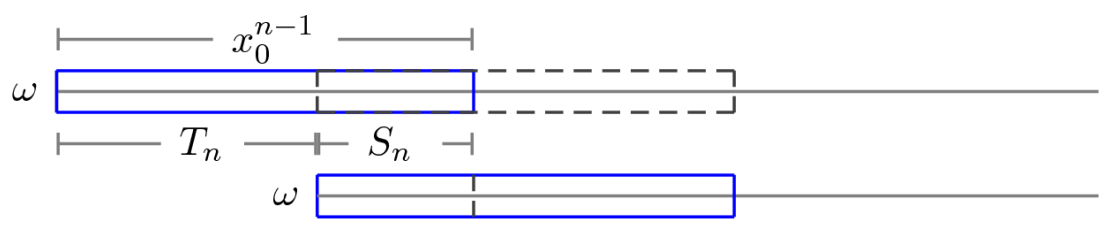

Figura 1.3: Relação entre $S_{n}$ e $T_{n}$ com $T_{n}<n$.

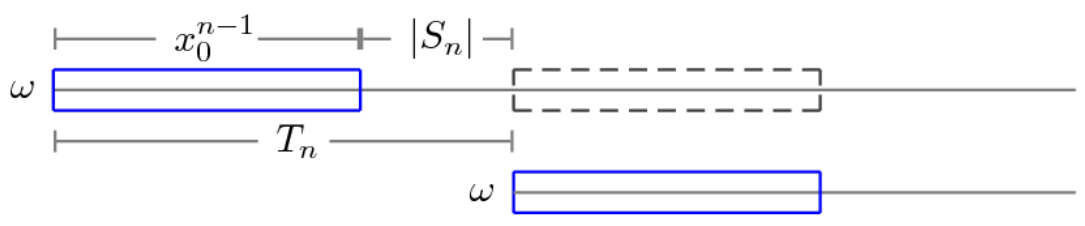

Figura 1.4: Relação entre $S_{n}$ e $T_{n} \operatorname{com} T_{n} \geq n$.

Nas figuras anteriores, a palavra $\omega$ é transladada um número de passos à direita até ela começar de novo. Esse número de passos será nosso $T_{n}$ se ele for o ínfimo entre todas as realizações do processo. Nas figuras estamos supondo que $\omega$ garante o ínfimo. Na figura 1.3, $T_{n}$ é menor que $n, \operatorname{logo} S_{n}$ representa o tamanho da máxima sobreposição que apresenta a palavra, ou seja, $S_{n}$ é, neste caso, o tamanho máximo de uma subpalavra com a qual $x_{0}^{n-1}$ começa e termina. Na figura $1.4, T_{n}$ é maior que $n$ e o valor absoluto de $S_{n}$ representa a distância entre a palavra $x_{0}^{n-1}$ e sua nova aparição.

Exemplo 1.12. Consideremos a palavra $x_{1}^{6}=010101$ do Exemplo 1.10. Neste exemplo se mostra que $T_{6}(010101)=2$. Logo $S_{n}\left(x_{1}^{6}\right)=4$ e a palavra tem uma sobreposição de tamanho 4.

Exemplo 1.13. Usando o processo do Exemplo 1.11, consideremos a palavra $x_{1}^{4}=1012$. Se $T_{4}(1012)=5$, quer dizer que existe uma palavra $\omega$ no espaço amostral, que começa com 101201012 e que garante o infimo. Então $S_{4}(1012)=-1$ e seu valor absoluto representa a distância que existe entre a palavra e sua nova aparição.

\subsubsection{Classificação periódica das palavras}

Uma forma de entender melhor o problema do tempo de retorno é tentar identificar algum comportamento periódico nas palavras. Note que, quando temos uma palavra formada por "bloquinhos" que se repetem, temos um retorno iminente. A seguir, definiremos uma ferramenta fundamental deste trabalho:

Definição 1.14. Seja $0<k<n$. Definimos $B_{n}(k)$ como sendo o conjunto de todas as palavras de $\chi^{n}$ que são k-periódicas. Ou seja:

$$
B_{n}(k)=\left\{x_{1}^{n} \in \chi^{n}: x_{1}^{n}=(\underbrace{x_{1}, \ldots, x_{k}}_{1}, \underbrace{x_{1}, \ldots, x_{k}}_{2}, \ldots, \underbrace{x_{1}, \ldots, x_{k}}_{\lfloor n / k\rfloor}, \underbrace{x_{1}, \ldots, x_{r}}_{1})\right\} .
$$


Portanto, o conjunto $B_{n}(k)$ pode ser visto como o conjunto de todas as palavras que podem ser escritas como concatenação de $\lfloor n / k\rfloor$ palavras $x_{1}^{k} \in \chi_{1}^{k}$ com uma palavra $x_{1}^{r} \in \chi_{1}^{r}$. Aqui, sempre consideramos $r<k$, o resto da divisão de $n$ por $k$. Note que o tamanho da palavra não precisa necessariamente ser um múltiplo de $k$. Somente a palavra precisa apresentar certo "comportamento periódico". Falando de forma mais simples, a palavra é formada por "bloquinhos" de tamanho $k$, mais um bloquinho restante de tamanho $0 \leq r<k$.

Exemplo 1.15. Considere o conjunto de palavras de tamanho $n=3$ do alfabeto $\chi=\{0,1\}$. Denotaremos esse conjunto por $C_{3}$. Dai:

$$
C_{3}=\{000 ; 001 ; 010 ; 011 ; 100 ; 101 ; 110 ; 111\} .
$$

Além disso:

$$
\begin{aligned}
& B_{3}(1)=\{000 ; 111\} \\
& B_{3}(2)=\{000 ; 111 ; 101 ; 010\} \\
& B_{3}(3)=C_{3} .
\end{aligned}
$$

O seguinte lema, tomado de Abadi e Vaienti [7] e [8], será úteis na provas dos resultados principais no próximo capítulo.

Lema 1.16. Sejam $m$ e $n$ inteiros positivos tais que $m<n / 2$. Então,

$$
\bigcup_{j=1}^{m} B_{n}(j)=\bigcup_{j=\left\lceil\frac{m}{2}\right\rceil}^{m} B_{n}(j),
$$

onde $\lceil\cdot\rceil$ é a função maior inteiro.

A prova desse lema, está baseada no fato que $B_{n}(j) \subseteq B_{n}(m j)$ para $m \in \mathbb{N}$. Definiremos agora os conjuntos $R_{n}(k)$, que serão as palavras com uma sobreposição de tamanho $k$. O motivo para essa mudança é mera conveniência

Definição 1.17. Definimos o conjunto $R_{n}(k)$ como sendo o conjunto de palavras que tem uma sobreposição de tamanho $k$. Ou seja:

$$
R_{n}(k)=\left\{x_{1}^{n} \in \chi^{n}: x_{1}^{k}=x_{n-k+1}^{n}\right\} .
$$

Observação 1.18. Note que vale a seguinte relação de dualidade:

$$
R_{n}(k)=B_{n}(n-k)
$$

\subsection{Resultados Preliminares}

O nosso objetivo neste seção é apresentar os principais resultados, obtidos por Lambert em [19] e por Abadi \& Lambert em [5], que motivaram esta tese. Vamos expor os teoremas e proposições que serão generalizados no Capítulo 2 e que foram nosso objeto de estudo.

Vamos considerar um processo independente com distribuições marginais identicamente distribuídas no alfabeto $\chi$. Nessas condições, em [19] e em [5], os autores provaram a 
convergência em distribuição da função $S_{n}$ e encontraram o limite para a velocidade de convergência. Como consequência, obtiveram a convergência da esperança da função $S_{n}$.

Quando o processo que gera as palavras é independente e identicamente distribuído, $S_{n}$ tem uma interessante interpretação: para cada palavra, a função $S_{n}$ assume o valor do maior tamanho possível de uma sobreposição que essa palavra pode ter com uma cópia dela mesma transladada. Se não houver sobreposição, a função assume o valor de zero, pois neste caso, a função $S_{n}$ não toma valores negativos. Isto é porque o processo independente tem gramática completa.

\subsubsection{Distribuição limite de $S_{n}$}

Vamos começar com o teorema apresentado em [9] e em [25]. Este teorema nos diz que, se o alfabeto for finito, o tempo de retorno $T_{n}$ cresce na mesma velocidade que $n$ quase certamente.

Teorema 1.19. Seja $\mu$ uma medida ergódica com respeito à transformação shift no espaço $\chi^{\mathbb{N}}$. Suponha também que o sistema tem entropia estritamente positiva com respeito a uma partição finita ou contável e que satisfaz a propriedade de especificação [9; 26]. Então,

$$
\lim _{n \rightarrow \infty} \frac{T_{n}}{n}=1 \quad \mu-q . c .
$$

A propriedade de especificação ou sistema fracamente especificado é uma propriedade topológica que nasce no desejo de aproximar simultaneamente (até $\epsilon$ ) duas palavras por uma órbita periódica. Sem ela, $\lim _{n \rightarrow \infty} T_{n} / n \geq c \mu$-q.c, $\operatorname{com} c \geq 1$. Para maior informação sobre a propriedade de especificação, remitimos ao leitor a [9]. Se o alfabeto for finito o processo satisfaz a propriedade de especificação [26].

Observação 1.20. Seja $S_{n}: \chi^{n} \rightarrow \mathbb{Z}$ como foi construído no na seção 1.1.2. Se o processo satisfaz a propriedade de especificação, então

$$
\lim _{n \rightarrow \infty} \frac{S_{n}}{n}=0 \quad \mu-q . c .
$$

Logo, nós concluímos que $S_{n}$ apresenta um comportamento assintótico melhor do que o comportamento assintótico apresentado por $T_{n}$. Eis mais uma razão para estudar o tempo de retorno através da função $S_{n}$.

Para entender melhor os próximos resultados, damos a seguinte notação: seja $\chi$ o alfabeto do processo. Para cada $a_{i} \in \chi$, sejam $\mu\left(a_{i}\right)=p_{i}$, então denotamos por $m_{l}$ a seguinte expressão

$$
m_{l}=\sum_{i=1}^{|\chi|} p_{i}^{l}
$$

Finalmente, chegamos ao teorema principal deste capítulo que diz, essencialmente, que existe uma distribuição limite para $S_{n}$ e que é semelhante a uma distribuição geométrica mais um termo de correção, e que a convergência para essa distribuição limite ocorre com taxa exponencial. 
Teorema 1.21. Seja $\mu$ uma medida produto em $\chi^{\mathbb{N}}$ com marginais identicamente distribuídas. Então, para todo inteiro positivo $k$, temos que

$$
\lim _{n \rightarrow \infty} \mu\left(S_{n} \geq k\right)=m_{2}^{k}+a_{k}
$$

onde $a_{k}=\sum_{i=k+1}^{\infty} \mu\left(R_{2 i}(i) \backslash \bigcup_{j=k}^{i-1} R_{2 i}(j)\right)$.

O termo $a_{k}$ seria a soma das medidas daquelas palavras duplas que têm uma sobreposição de tamanho igual à mitade da palavra mas não têm ninhuma sobreposição de tamanho menor.

As seguintes proposições apresentam limitantes para o termo de correcção $a_{k}$.

Proposição 1.22. Sob as condições do Teorema 1.21, temos que

$$
a_{k} \leq \frac{m_{2}^{k+1}}{1-m_{2}}-\frac{m_{4}^{k}}{1-m_{2}} .
$$

O limitante que dá a proposição anterior não estabelece qual termo entre $m_{2}^{k}$ e $a_{k}$ é maior, mas a seguinte proposição, mostra que de fato ambas podem acontecer.

Proposição 1.23. Sob as condições do Teorema 1.21, temos que

$$
a_{k} \geq \frac{m_{2}^{k+1}}{1-m_{2}}-\frac{m_{4}^{k}}{1-m_{4}} \frac{1+m_{2}}{1-m_{2}} .
$$

Além disso,

a. Se $m_{2} \leq 1 / 2$ ent $\tilde{a} o, m_{2}^{k}>a_{k}$,

b. Se $m_{2}>1 / 2$, então

i. $a_{1}<m_{2}$

ii. Existe para algum $k_{0}>0$, para o qual $a_{k}>m_{2}^{k}$ para todo $k>k_{0}$.

Exemplo 1.24. Considere a medida produto uniforme sobre o alfabeto finito $\chi=\{1,2, \ldots, s\}$. Então, $m_{i}=1 / s^{i-1}$. As desigualdades dadas nas Proposições 1.22 e 1.23 ficam

$$
\begin{gathered}
a_{k} \leq \frac{s^{2 k-1}-1}{s^{3 k-1}(s-1)} e \\
a_{k} \geq \frac{1}{s^{k}(s-1)}-\frac{s+1}{s-1} \frac{1}{s^{3 k-2}\left(s^{3}-1\right)}
\end{gathered}
$$

e pela Proposição 1.23a. $m_{2}$ é sempre o termo dominante.

Exemplo 1.25. Vamos considerar o caso Bernoulli com $\chi=\{0,1\}$. Sejam $p_{1}=p$ e $p_{0}=$ $1-p$. Logo $m_{i}=p^{i}+(1-p)^{i}$, particularmente $m_{2}=p^{2}+(1-p)^{2}$. Aplicando as fórmulas dadas nas Proposições 1.22 e 1.23 obtém-se que

$$
a_{k}=O\left(\frac{\left(p^{2}+(1-p)^{2}\right)^{k+1}}{2 p(1-p)}\right)
$$

e se $p \neq 1 / 2$, então $m_{2}^{k}>1 / 2$ e pela parte $b$, item ii. da Proposição 1.23 existe $k_{0} \in \mathbb{N}$ para o qual $m_{2}<a_{k}$ para todo $k>k_{0}$. 


\subsubsection{Convergência de $\mathbb{E}\left(S_{n}\right)$}

Vamos analisar agora o comportamento assintótico de $\mathbb{E}\left(S_{n}\right)$ quando o processo que gera as palavras é independente com distribuições marginais identicamente distribuídas. A dificuldade na abordagem do cálculo de $\mathbb{E}\left(S_{n}\right)$ radica no não conhecimento da distribuição explicíta de $S_{n}$. Por isso é preciso procurar limitantes como foi feito por Lambert em [19] cujos resultados daremos a seguir.

Começamos com um corolário do Teorema 1.21 onde se obtém a convergência da esperança de $S_{n}$ para um valor $Q$.

Corolário 1.26. Seja $Q=\lim _{n \rightarrow \infty} \mathbb{E}\left(S_{n}\right)$. Então, existem $C$ constante positiva e $0<\epsilon<1$, tal que

$$
\left|\mathbb{E}\left(S_{n}\right)-Q\right| \leq C \epsilon^{n}
$$

O corolário anterior mostra uma convergência exponencialmente rápida ao valor desconhecido $Q$. No seguinte corolário vamos ver limitantes para este valor.

Corolário 1.27. Seja $Q=\lim _{n \rightarrow \infty} \mathbb{E}\left(S_{n}\right)$. Então,

$$
\frac{m_{2}}{1-m_{2}} \leq Q \leq \frac{m_{2}}{\left(1-m_{2}\right)^{2}}
$$

No Capítulo 2, nós calculamos limitantes mais eficientes para $Q$ e também para $Q^{2}=$ $\lim _{n \rightarrow \infty} \mathbb{E}\left(S_{n}^{2}\right)$.

\subsection{3 $\mathbb{E}\left(S_{n}\right)$ e a entropia do processo Bernoulli}

Uma pergunta interessante é a relação entre a entropia do processo e $\mathbb{E}\left(S_{n}\right)$. Em [19] o autor conjectura que, de fato, esta relação existe no caso de um processo Bernoulli. Para isso, o autor considerou o alfabeto com dois símbolos, $\chi=\{0,1\}$ tal que $p_{1}=p$ e $p_{0}=1-p$.

Guilherme Ludwig, sob a orientação do professor Miguel Abadi, simulou amostras de palavras de tamanho 250. Em seguida calculou $\mathbb{E}\left(S_{n}\right)$ para cada caso. Foi usada a entropia de Shannon, $H(p)=-(p \ln p+(1-p) \ln (1-p))$, para a comparação nas simulações. O gráfico que eles obtiveram sugere que $\mathbb{E}\left(S_{n}\right)$ tem uma relação inversa com a entropia do processo.

Note que quando $p$ se aproxima a 0 ou 1 , a esperança de $S_{n}$ é máxima e a entropia do processo tende a 0 . Nesse caso o nível de complexidade do processo diminuiu e o tempo de retorno das sequências é pequeno. Isto se deve ao fato de um mesmo símbolo ocorrer com probabilidade grande o que faz que a esperança cresça.

\subsubsection{Não convergência em probabilidade}

Abadi \& Lambert [5] provaram que $S_{n}$ não converge em probabilidade quando $n$ tende a infinito. Para evitar casos triviais, os autores consideraram $\mu\left(a_{i}\right)<1$, para todo $a_{i} \in \chi$.

Proposição 1.28. Sob as condiçoẽs do Teorema 1.21, não existe uma variável aleatória $S$ definida sobre $\chi^{\mathbb{N}}$ tal que $S_{n}$ converge a $S$ em probabilidade. 


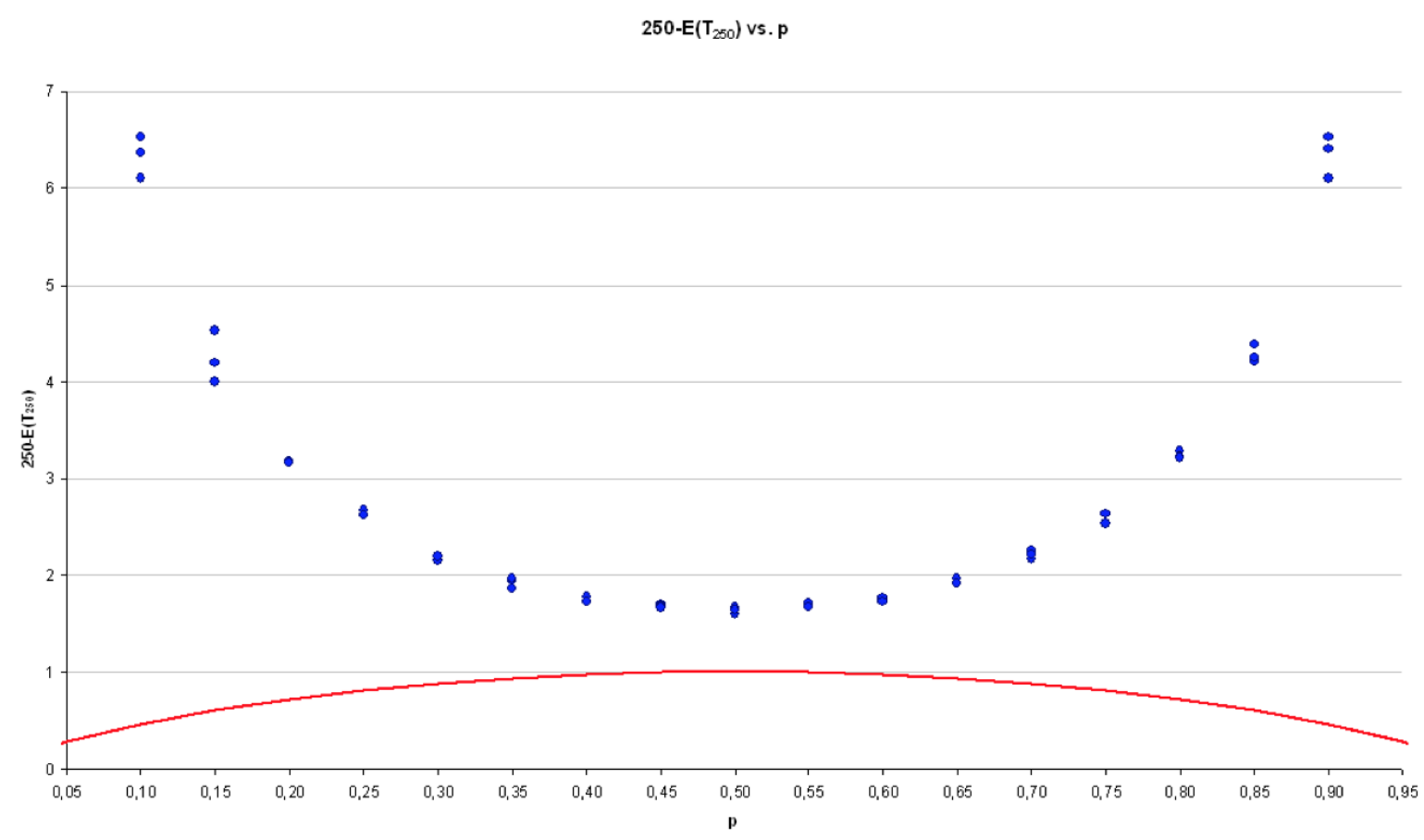

Figura 1.5: $\mathbb{E}\left(S_{n}\right)$ vs. Entropia

Embora a proposição esteja provada usando um processo independente, é valida para processos mixing, em particular para $\beta$-mixing, porque se $\mu\left(x_{i}^{j} \cap x_{j+n}^{m}\right)=\mu\left(x_{i}^{j}\right) \mu\left(x_{j+n}^{m}\right)$ para quaisquer $i, j, m \in \mathbb{N}$, então $\beta(n)=0$ para todo $n$. Ou seja, um processo independente é um processo $\beta$-mixing onde $\beta(n)=0$ para todo $n$. 


\section{Capítulo 2}

\section{Resultados obtidos}

Neste capítulo vamos generalizar os resultados expostos no capítulo anterior. A generalização é feita enfraquecendo a hipótese de independência entre as variáveis geradoras do processo, as quais têm uma dependência do tipo $\beta$-mixing definida no Capítulo 1.

\subsection{Distribuição limite de $S_{n}$}

Vamos começar apresentando o teorema principal desta seção. Este teorema diz, essencialmente, que sob hipóteses convenientes para os coeficientes $\beta$-mixing, $S_{n}$ converge en distribuição quando $n$ diverge.

Teorema 2.1. Seja $\mu$ uma medida de probabilidade definida em $\chi^{\mathbb{N}}$ para um processo $\beta$ mixing com gramática completa. Considere $k \in \mathbb{N}$ tal que $n \geq 4 k$. Então

$$
\left|\mu\left(S_{n} \geq k\right)-\mathbb{E}\left(\mu\left(X_{1}^{k}\right)\right)-a_{k}\right| \leq K_{\rho} \sum_{i=\lceil n / 8\rceil}^{\infty} \varepsilon_{\beta}(i)
$$

onde

$$
\rho=\max _{x \in \chi} \mu(x)
$$

$K_{\rho}$ é uma constante que só depende de $\rho$,

$$
\varepsilon_{\beta}(i)=\inf _{1 \leq m<i}\left(\rho^{m}+\tilde{\beta}_{m}(i)\right)
$$

$m \in \mathbb{N}, \tilde{\beta}_{m}(i)=2 \beta_{m}(i)=2 \beta(\lfloor i / m\rfloor-1), e$

$$
a_{k}=\sum_{i=k+1}^{\infty} \sum_{\omega \notin \cup_{j=k}^{i-1} R_{i}(j)} \mu^{2}(\omega)
$$

Se, além da hipótese anterior, o processo $\beta$-mixing é tal que ln $i \beta(i)$ é somável, então

$$
\lim _{n \rightarrow \infty} \sum_{i=\lceil n / 4\rceil}^{\infty} \varepsilon_{\beta}(i)=0,
$$


o que implica que

$$
\lim _{n \rightarrow \infty} \mu\left(S_{n} \geq k\right)=\mathbb{E}\left(\mu\left(X_{1}^{k}\right)\right)+a_{k} .
$$

O teorema nós diz que o limite da probabilidade de $S_{n} \geq k$, com $k>0$ converge à esperança da medida das palavas de tamanho $k$ mais um termo de correção $a_{k}$. Este termo $a_{k}$ depende da medida daquelas palavras que pertencem a $R_{n}(j) \operatorname{com} j>k$ mas não pertencem a $R_{n}(k)$, ou seja, são aquelas palavras que têm uma sobrepocisão de tamanho maior a $k$ mas não têm uma sobrepocisão de tamanho $k$.

Vamos mostrar que o termo $\mathbb{E}\left(\mu\left(X_{1}^{k}\right)\right)$ do Teorema anterior corresponde ao termo $m_{2}^{k}$ do Teorema 1.21 no caso independente, mostrando assim, a generalização feita.

Observação 2.2. Se o processo que gera as palavras é independente, então

$$
\mathbb{E}\left(\mu\left(X_{1}^{k}\right)\right)=m_{2}^{k}
$$

Demonstração. Pela definição de esperança de uma variável aleatória,

$$
\begin{aligned}
\mathbb{E}\left(\mu\left(X_{1}^{k}\right)\right) & =\sum_{x_{1}^{k} \in \chi^{k}} \mu^{2}\left(x_{1}^{k}\right) \\
& =\sum_{x_{1} x_{2} \ldots x_{k}} \mu^{2}\left(x_{1}\right) \mu^{2}\left(x_{2}\right) \ldots \mu^{2}\left(x_{k}\right) \\
& =\left(\sum_{x \in \chi} \mu^{2}(x)\right)^{k} \\
& =m_{2}^{k},
\end{aligned}
$$

onde as últimas linhas foram obtidas pela independência do processo e pela definição de $m_{l}$ dada em (1.7).

As seguintes proposições dão limitantes para o termo $a_{k}$.

Proposição 2.3. Sob as hipóteses do Teorema 2.1, um limitante superior para $a_{k}$ é

$$
a_{k} \leq \sum_{i=k+1}^{\infty} \mathbb{E}\left(\mu\left(X_{1}^{i}\right)\right)-\sum_{i=k+1}^{\infty} \sum_{\omega \in R_{i}(k)} \mu^{2}(\omega) .
$$

Proposição 2.4. Sob as hipóteses do Teorema 2.1, um limite inferior para $a_{k}$ é

$$
a_{k} \geq \sum_{i=k+1}^{\infty} \mathbb{E}\left(\mu\left(X_{1}^{i}\right)\right)-\sum_{i=k+1}^{\infty} \sum_{j=k}^{i-1} \sum_{\omega \in R_{i}(j)} \mu^{2}(\omega) .
$$

As provas do Teorema 2.1, e as Proposições 2.3 e 2.4, serão desenvolvidas a partir de três lemas, enunciados e provados a seguir.

Lema 2.5. Seja $\left\{X_{n}\right\}_{n \in \mathbb{N}}$ um processo $\alpha$-mixing definido em $\chi^{\mathbb{N}}$. Então,

$$
\mu\left(x_{1}^{n}\right) \leq \inf _{1 \leq m<n}\left(\rho^{m+1}+\frac{\alpha(\lfloor n / m\rfloor-1)}{1-\rho}\right),
$$

onde $m \in \mathbb{N},\lfloor\cdot\rfloor$ denota a função parte inteira e $\rho$ como foi definido em (2.2). 
Demonstração. Esta prova está baseada no Lema 1 de [14] e no Lema 18 de [2].

Para qualquer $1 \leq m<n$, é válido que $n=m\left\lfloor\frac{n}{m}\right\rfloor+r_{m}$, onde $r_{m}$ é o resto da divisão de $n$ por $m$.

Para qualquer palavra de tamanho $n$, digamos $x_{1}^{n}$, temos que

$$
\mu\left(x_{1}^{n}\right) \leq \mu\left(X_{1}=x_{1}, X_{\lfloor n / m\rfloor+1}^{n}=x_{\lfloor n / m\rfloor+1}^{n}\right),
$$

onde a desigualdade foi obtida pela remoção do bloco $x_{2}^{\lfloor n / m\rfloor}$ de $x_{1}^{n}$. A propriedade $\alpha$-mixing permite afirmar que

$$
\mu\left(X_{1}=x_{1}, X_{\lfloor n / m\rfloor+1}^{n}=x_{\lfloor n / m\rfloor+1}^{n}\right) \leq \mu\left(x_{1}\right) \mu\left(x_{\lfloor n / m\rfloor+1}^{n}\right)+\alpha(\lfloor n / m\rfloor-1) .
$$

Vamos remover agora o bloco $x_{\lfloor n / m\rfloor+2}^{2\lfloor n / m\rfloor}$ de $x_{\lfloor n / m\rfloor+1}^{n}$ obtendo que

$$
\mu\left(x_{\lfloor n / m\rfloor+1}^{n}\right) \leq \mu\left(X_{\lfloor n / m\rfloor+1}=x_{\lfloor n / m\rfloor+1}, X_{2\lfloor n / m\rfloor+1}^{n}=x_{2\lfloor n / m\rfloor+1}^{n}\right) .
$$

e como o processo é $\alpha$-mixing, temos que

$$
\mu\left(X_{\lfloor n / m\rfloor+1}=x_{\lfloor n / m\rfloor+1}, X_{2\lfloor n / m\rfloor+1}^{n}=x_{2\lfloor n / m\rfloor+1}^{n}\right) \leq \mu\left(x_{\lfloor n / m\rfloor+1}\right) \mu\left(x_{2\lfloor n / m\rfloor+1}^{n}\right)+\alpha(\lfloor n / m\rfloor-1) .
$$

Juntando as equações (2.6), (2.7), (2.8) e (2.9), concluímos que

$$
\mu\left(x_{1}^{n}\right) \leq \mu\left(x_{1}\right) \mu\left(x_{\lfloor n / m\rfloor+1}\right) \mu\left(x_{2\lfloor n / m\rfloor+1}^{n}\right)+\alpha(\lfloor n / m\rfloor-1)\left(1+\mu\left(x_{1}\right)\right) .
$$

Repetindo iterativamente a propriedade $\alpha$-mixing para as medidas $\mu\left(x_{i\lfloor n / m\rfloor+1}^{n}\right)$ com $i=$ $2, \ldots, m$, obtemos que

$$
\mu\left(x_{1}^{n}\right) \leq \prod_{i=0}^{m} \mu\left(x_{i\lfloor n / m\rfloor+1}\right)+\alpha(\lfloor n / m\rfloor-1) \sum_{i=0}^{m-1} \prod_{k=0}^{i} \mu\left(x_{k\lfloor n / m\rfloor+1}\right) .
$$

Seja $\rho=\max _{x \in \chi} \mu(x)$. Assim,

$$
\begin{aligned}
\mu\left(x_{1}^{n}\right) & \leq \rho^{m+1}+\alpha(\lfloor n / m\rfloor-1) \sum_{i=0}^{m-1} \rho^{i} \\
& \leq \rho^{m+1}+\frac{\alpha(\lfloor n / m\rfloor-1)}{1-\rho} .
\end{aligned}
$$

Isso finaliza nossa prova.

Lema 2.6. Seja $\mu$ uma medida de probabilidade definida em $\chi^{\mathbb{N}}$ para um processo $\alpha$-mixing. Então,

$$
\mathbb{E}\left(\mu\left(X_{1}^{n}\right)\right) \leq \inf _{1 \leq m<n}\left(\rho^{m+1}+\frac{\alpha(\lfloor n / m\rfloor-1)}{1-\rho}\right)
$$

onde $m \in \mathbb{N}$. 
Demonstração. Pela definição de esperança de uma variável aleatória, temos que

$$
\begin{aligned}
\mathbb{E}\left(\mu\left(X_{1}^{n}\right)\right) & =\sum_{w \in \chi^{n}} \mu^{2}(w) \\
& \leq \max _{\omega \in \chi^{n}} \mu(\omega) .
\end{aligned}
$$

Vamos denotar por $p_{n}=\max _{\omega \in \chi^{n}} \mu(\omega)$.

Podemos aplicar o Lema $2.5 \mathrm{em} p_{n}$, porque o limitante superior que ele fornece não depende das palavras $\omega$ de tamanho $n$, logo, em particular, o lema vale para a palavra de tamanho $n$ com medida máxima. Assim,

$$
p_{n} \leq \inf _{1 \leq m<n}\left(\rho^{m+1}+\frac{\alpha(\lfloor n / m\rfloor-1)}{1-\rho}\right) .
$$

E substituindo esta última expressão na inequação (2.13), chegamos a nosso resultado.

Observação 2.7. Se o processo $\left\{X_{n}\right\}_{n \in \mathbb{N}}$ é $\beta$-mixing então ele é também $\alpha$-mixing, mediante a inequação $2 \alpha(n) \leq \beta(n)$ [11], e portanto

i. A expressão (2.5) do Lema 2.5 para processos $\beta$-mixing seria,

$$
\mu\left(x_{1}^{n}\right) \leq \inf _{1 \leq m<n}\left(\rho^{m+1}+\frac{\beta(\lfloor n / m\rfloor-1)}{2(1-\rho)}\right) .
$$

ii. A expressão (2.12) do Lema 2.6 para processos $\beta$-mixing é

$$
\mathbb{E}\left(\mu\left(X_{1}^{n}\right)\right) \leq \inf _{1 \leq m<n}\left(\rho^{m+1}+\frac{\beta_{m}(n)}{2(1-\rho)}\right) .
$$

Lema 2.8. Sejam $\mu$ uma medida de probabilidade definida em $\chi^{\mathbb{N}}$ para um processo $\beta$-mixing $e 0<c<1$ uma constante. Então,

$$
\mu\left(\bigcup_{j=1}^{\lfloor c n\rfloor} B_{n}(j)\right) \leq K_{c, \rho} \sum_{j=\left\lfloor c^{\prime} n\right\rfloor}^{\lfloor c n\rfloor} \inf _{1 \leq m<j}\left(\rho^{m}+\tilde{\beta}_{m}(j)\right)
$$

onde $K_{c, \rho}$ é uma constante que depende de c e $\rho$ e $c^{\prime}=\min \{c / 2,(1-c) / 2\}$.

Demonstração. Começamos usando o Lema 1.16 para afirmar que

$$
\begin{aligned}
\mu\left(\bigcup_{j=1}^{\lfloor c n\rfloor} B_{n}(j)\right) & =\mu\left(\bigcup_{j=\lceil\lfloor c n\rfloor / 2\rceil}^{\lfloor c n\rfloor} B_{n}(j)\right) \\
& \leq \sum_{j=\lceil\lfloor c n\rfloor / 2\rceil}^{\lfloor c n\rfloor} \mu\left(B_{n}(j)\right) .
\end{aligned}
$$

Vamos nos concentrar em $\mu\left(B_{n}(j)\right)$.

$$
\mu\left(B_{n}(j)\right)=\sum_{\omega \in B_{n}(j)} \mu(\omega) .
$$


Se $\omega \in B_{n}(j)$, então $\omega$ esta formado pela concatenação de $\lfloor n / j\rfloor$ blocos de tamanho $j$, mais um último bloco de tamanho $r<j$, o qual é o resto da divisão de $n$ por $j$. Ou seja, dado que $n=j\left\lfloor\frac{n}{j}\right\rfloor+r$, podemos dividir $\omega$ em subpalavras $x_{1}^{j}$ concatenadas assim,

$$
\omega=\underbrace{x_{1}^{j} x_{1}^{j} \ldots x_{1}^{j}}_{\left\lfloor\frac{n}{j}\right\rfloor \text { vezes }} x_{1}^{r} .
$$

Além do anterior, $x_{1}^{j}=x_{1}^{r} x_{r+1}^{j}$, ou seja, o bloco $x_{1}^{r}$ é o começo de todos os blocos $x_{1}^{j}$. Assim, quando nós conhecemos $x_{1}^{j}$, também conheceremos $x_{1}^{r}$.

Dividiremos a prova em dois casos, quando $0<c \leq 1 / 2$ e quando ocorre o contrário, $1 / 2<c<1$. Suponha que $0<c \leq 1 / 2$. Nosso objetivo será aplicar, iterativamente, a propriedade $\beta$-mixing para limitar $\mu(\omega)$. Para isso, começamos removendo de $\omega$ o bloco $x_{j+1}^{2 j}$, resultando em

$$
\mu(\omega) \leq \mu\left(X_{1}^{j}=x_{1}^{j}, X_{2 j+1}^{n}=x_{2 j+1}^{n}\right) .
$$

Vamos denotar $\tilde{\beta}(j)=2 \beta(j)$. A propriedade $\beta$-mixing do processo diz que

$$
\sum_{x_{1}^{j}, x_{2 j+1}^{n}}\left|\mu\left(X_{1}^{j}=x_{1}^{j}, X_{2 j+1}^{n}=x_{2 j+1}^{n}\right)-\mu\left(x_{1}^{j}\right) \mu\left(x_{2 j+1}^{n}\right)\right| \leq \tilde{\beta}(j) .
$$

Mas, a palavra

$$
x_{2 j+1}^{n}=\underbrace{x_{1}^{j} x_{1}^{j} \ldots x_{1}^{j}}_{\left\lfloor\frac{n}{j}\right\rfloor-2 \text { vezes }} x_{1}^{r},
$$

isso implica que nós só temos que somar com respeito a $x_{1}^{j}$, obtendo que

$$
\begin{aligned}
\sum_{x_{1}^{j}} \mid \mu\left(X_{1}^{j}=x_{1}^{j}, X_{2 j+1}^{n}=x_{2 j+1}^{n}\right) & -\mu\left(x_{1}^{j}\right) \mu\left(x_{2 j+1}^{n}\right) \mid \\
& \leq \sum_{x_{1}^{j}, x_{2 j+1}^{n}}\left|\mu\left(X_{1}^{j}=x_{1}^{j}, X_{2 j+1}^{n}=x_{2 j+1}^{n}\right)-\mu\left(x_{1}^{j}\right) \mu\left(x_{2 j+1}^{n}\right)\right| \\
& \leq \tilde{\beta}(j)
\end{aligned}
$$

O que nos leva a afirmar que,

$$
\sum_{x_{1}^{j}} \mu\left(X_{1}^{j}=x_{1}^{j}, X_{2 j+1}^{n}=x_{2 j+1}^{n}\right) \leq \sum_{x_{1}^{j}} \mu\left(x_{1}^{j}\right) \mu\left(x_{2 j+1}^{n}\right)+\tilde{\beta}(j) .
$$

Usando a equações (2.18) e (2.19) concluímos que

$$
\sum_{x_{1}^{j}} \mu(\omega) \leq \sum_{x_{1}^{j}} \mu\left(x_{1}^{j}\right) \mu\left(x_{2 j+1}^{n}\right)+\tilde{\beta}(j) .
$$


Observe que $\mu\left(x_{1}^{j}\right) \leq \max _{\omega \in \chi^{j}} \mu(\omega)=p_{j}$, logo,

$$
\begin{aligned}
\sum_{x_{1}^{j}} \mu\left(x_{1}^{j}\right) \mu\left(x_{2 j+1}^{n}\right) & \leq \max _{\omega \in \chi^{j}} \mu(\omega) \sum_{x_{1}^{j}} \mu\left(x_{2 j+1}^{n}\right) \\
& =p_{j} \sum_{x_{1}^{j}} \mu\left(x_{2 j+1}^{n}\right),
\end{aligned}
$$

e portanto, substituindo (2.21) na desigualdade (2.20),

$$
\sum_{x_{1}^{j}} \mu(\omega) \leq p_{j} \sum_{x_{1}^{j}} \mu\left(x_{2 j+1}^{n}\right)+\tilde{\beta}(j) .
$$

Agora, removemos o bloco $x_{3 j+1}^{4 j}$ de $x_{2 j+1}^{n}$ e obtemos

$$
\mu\left(x_{2 j+1}^{n}\right) \leq \mu\left(X_{2 j+1}^{3 j}=x_{1}^{j}, X_{4 j+1}^{n}=x_{4 j+1}^{n}\right) .
$$

Segundo a propriedade $\beta$-mixing,

$$
\begin{aligned}
\sum_{x_{1}^{j}} \mid \mu\left(X_{2 j+1}^{3 j}=x_{1}^{j}, X_{4 j+1}^{n}=x_{4 j+1}^{n}\right) & -\mu\left(x_{1}^{j}\right) \mu\left(x_{4 j+1}^{n}\right) \mid \\
& \leq \sum_{x_{1}^{j}, x_{4 j+1}^{n}}\left|\mu\left(X_{1}^{j}=x_{1}^{j}, X_{2 j+1}^{n}=x_{4 j+1}^{n}\right)-\mu\left(x_{1}^{j}\right) \mu\left(x_{4 j+1}^{n}\right)\right| \\
& \leq \tilde{\beta}(j) .
\end{aligned}
$$

Isso nós permite afirmar que,

$$
\sum_{x_{1}^{j}} \mu\left(X_{2 j+1}^{3 j}=x_{1}^{j}, X_{4 j+1}^{n}=x_{4 j+1}^{n}\right) \leq \sum_{x_{1}^{j}} \mu\left(x_{1}^{j}\right) \mu\left(x_{4 j+1}^{n}\right)+\tilde{\beta}(j) .
$$

Novamente,

$$
\begin{aligned}
\sum_{x_{1}^{j}} \mu\left(x_{1}^{j}\right) \mu\left(x_{4 j+1}^{n}\right) & \leq \max _{\omega \in \chi^{j}} \mu(\omega) \sum_{x_{1}^{j}} \mu\left(x_{4 j+1}^{n}\right) \\
& =p_{j} \sum_{x_{1}^{j}} \mu\left(x_{4 j+1}^{n}\right),
\end{aligned}
$$

e substituindo a inequação (2.23) na inequação (2.22), resulta que

$$
\begin{aligned}
\sum_{x_{1}^{j}} \mu(\omega) & \leq p_{j}^{2} \sum_{x_{1}^{j}} \mu\left(x_{4 j+1}^{n}\right)+\tilde{\beta}(j) p_{j}+\tilde{\beta}(j) \\
& <p_{j}^{2} \sum_{x_{1}^{j}} \mu\left(x_{4 j+1}^{n}\right)+2 \tilde{\beta}(j) .
\end{aligned}
$$

Se continuarmos removendo, iterativamente, todos os blocos $x_{(i-1) j+1}^{i j}$ com $i$ par desde 6 até $\left\lfloor\frac{n}{j}\right\rfloor$ quando $\left\lfloor\frac{n}{j}\right\rfloor$ for par e aplicando a propriedade $\beta$-mixing, também iterativamente, 
teríamos que

$$
\sum_{x_{1}^{j}} \mu(\omega)<p_{j}^{k_{n, j}^{\prime}}+\left(k_{n, j}^{\prime}-1\right) \tilde{\beta}(j),
$$

onde $k_{n, j}^{\prime}=\lceil\lfloor n / j\rfloor / 2\rceil$. E se $\lfloor n / j\rfloor$ for ímpar, removemos os blocos $x_{(i-1) j+1}^{i j}$ com $i$ par desde 6 até $\left\lfloor\frac{n}{j}\right\rfloor-1$ e usando repetidamente a propriedade $\beta$-mixing, obtemos

$$
\sum_{x_{1}^{j}} \mu(\omega)<p_{j}^{k_{n, j}}+\left(k_{n, j}-1\right) \tilde{\beta}(j)
$$

onde $k_{n, j}=\lfloor\lfloor n / j\rfloor / 2\rfloor$. Em ambos casos,

$$
\sum_{x_{1}^{j}} \mu(\omega) \leq p_{j}^{k_{n, j}}+k_{n, j} \tilde{\beta}(j)
$$

É importante ressaltar aqui que como foi suposto $0<c \leq 1 / 2,1 \leq k_{n, j} \leq\lfloor 1 / c\rfloor$. Logo é verdade que,

$$
\mu\left(B_{n}(j)\right)<p_{j}+\left\lfloor\frac{1}{c}\right\rfloor \tilde{\beta}(j) .
$$

Agora, vamos limitar superiormente a quantidade $p_{j}$. Usando a equação (2.15), pois o nosso processo por hipótese é $\beta$-mixing, fica que

$$
p_{j} \leq \inf _{1 \leq m<j}\left(\rho^{m+1}+\frac{\beta_{m}(j)}{2(1-\rho)}\right)=C_{\rho} \inf _{1 \leq m<j}\left(\rho^{m}+\beta_{m}(j)\right),
$$

onde $C_{\rho}$ é uma constante que depende de $\rho$. Logo, substituindo a última desigualdade em (2.24), chegamos a

$$
\mu\left(B_{n}(j)\right)<C_{\rho} \inf _{1 \leq m<j}\left(\rho^{m}+\beta_{m}(j)\right)+\left\lfloor\frac{1}{c}\right\rfloor \tilde{\beta}(j) .
$$

Como a função $\beta$ é decrescente, $\tilde{\beta}(j) \leq \tilde{\beta}_{m}(j)$ e $\beta_{m}(j)<\tilde{\beta}_{m}(j)$ para todo $m<j$, e portanto

$$
\mu\left(B_{n}(j)\right)<C_{c, \rho} \inf _{1 \leq m<j}\left(\rho^{m}+\beta_{m}(j)\right),
$$

onde $C_{c, \rho}$ é uma constante que depende de $c$ e $\rho$. Somando $j$ desde $\lceil\lfloor c n\rfloor / 2\rceil$ até $\lfloor c n\rfloor$, temos que

$$
\sum_{j=\lceil\lfloor c n\rfloor / 2\rceil}^{\lfloor c n\rfloor} \mu\left(B_{n}(j)\right)<\sum_{j=\lceil\lfloor c n\rfloor / 2\rceil}^{\lfloor c n\rfloor} \inf _{1 \leq m<j}\left(\rho^{m}+\beta_{m}(j)\right) .
$$

Vamos estudar agora o segundo caso da prova, quando $1 / 2<c<1$. Neste caso, podemos partir o somatório em dois, assim:

$$
\sum_{j=\lceil\lfloor c n\rfloor / 2\rceil}^{\lfloor c n\rfloor} \mu\left(B_{n}(j)\right)=\sum_{j=\lceil\lfloor c n\rfloor / 2\rceil}^{\lfloor n / 2\rfloor} \mu\left(B_{n}(j)\right)+\sum_{j=\lfloor n / 2\rfloor+1}^{\lfloor c n\rfloor} \mu\left(B_{n}(j)\right) .
$$

Note que $\lfloor 1 / c\rfloor=1$ porque $c>1 / 2$, então pela desigualdade (2.24), o primeiro somatório 
da equação anterior é

$$
\sum_{j=\lceil\lfloor c n\rfloor / 2\rceil}^{\lfloor n / 2\rfloor} \mu\left(B_{n}(j)\right)<\sum_{j=\lceil\lfloor c n\rfloor / 2\rceil}^{\lfloor n / 2\rfloor}\left(p_{j}+\tilde{\beta}(j)\right)
$$

Levando em consideração a inequação (2.25), temos que

$$
\sum_{j=\lceil\lfloor c n\rfloor / 2\rceil}^{\lfloor n / 2\rfloor} \mu\left(B_{n}(j)\right) \leq C_{\rho} \inf _{1 \leq m<\lceil\lfloor c n\rfloor / 2\rceil} \sum_{j=\lceil\lfloor c n\rfloor / 2\rceil}^{\lfloor n / 2\rfloor}\left(p^{m}+\tilde{\beta}_{m}(j)\right)+\sum_{j=\lceil\lfloor c n\rfloor / 2\rceil}^{\lfloor n / 2\rfloor} \tilde{\beta}(j) .
$$

E dado que a função $\beta$ é decrescente, $\tilde{\beta}_{m}(j) \geq \tilde{\beta}(j)$,

$$
\sum_{j=\lceil\lfloor c n\rfloor / 2\rceil}^{\lfloor n / 2\rfloor} \mu\left(B_{n}(j)\right) \leq 2 C_{\rho} \sum_{j=\lceil\lfloor c n\rfloor / 2\rceil}^{\lfloor n / 2\rfloor} \inf _{1 \leq m<j}\left(p^{m}+\tilde{\beta}_{m}(j)\right) .
$$

Agora, vamos nos concentrar no segundo somatório à direita na inequação (2.27). Suponha que $j>n / 2$. Logo, a palavra $\omega$ é composta pela concatenação de um bloco de tamanho $j, x_{1}^{j}$, e outro de tamanho $r, x_{1}^{r}$. Mas, lembremos que $x_{1}^{r}$ esta determinado por $x_{1}^{j}$, pois $x_{1}^{j}=x_{1}^{r} x_{r+1}^{j}$. A partir disso, temos que

$$
\omega=x_{1}^{j} x_{1}^{r}=x_{1}^{r} x_{r+1}^{j} x_{1}^{r}=x_{1}^{\lfloor r / 2\rfloor} x_{\lfloor r / 2\rfloor+1}^{j} x_{1}^{r} .
$$

Como $c<1, n-j=r>0$, de fato $r \geq\lfloor n / 4\rfloor$. Removendo o bloco $x_{\lfloor r / 2\rfloor+1}^{j}$ de $\omega$, afirmamos que

$$
\mu(\omega) \leq \mu\left(x_{1}^{\lfloor r / 2\rfloor}, x_{1}^{r}\right)
$$

Usando a propriedade $\beta$-mixing

$$
\sum_{x_{1}^{r}}\left|\mu\left(x_{1}^{\lfloor r / 2\rfloor}, x_{1}^{r}\right)-\mu\left(x_{1}^{\lfloor r / 2\rfloor}\right) \mu\left(x_{1}^{r}\right)\right| \leq \tilde{\beta}(j-\lfloor r / 2\rfloor) .
$$

Logo,

$$
\sum_{x_{1}^{r}} \mu\left(x_{1}^{\lfloor r / 2\rfloor}, x_{1}^{r}\right) \leq \sum_{x_{1}^{r}} \mu\left(x_{1}^{\lfloor r / 2\rfloor}\right) \mu\left(x_{1}^{r}\right)+\tilde{\beta}(j-\lfloor r / 2\rfloor),
$$

o qual implica, pela expressão (2.29)

$$
\sum_{x_{1}^{r}} \mu(\omega) \leq \sum_{x_{1}^{r}} \mu\left(x_{1}^{\lfloor r / 2\rfloor}\right) \mu\left(x_{1}^{r}\right)+\tilde{\beta}(j-\lfloor r / 2\rfloor),
$$

ou seja que,

$$
\sum_{x_{1}^{j}} \mu(\omega) \leq p_{\lfloor r / 2\rfloor}+\tilde{\beta}(j-\lfloor r / 2\rfloor),
$$

onde $p_{\lfloor r / 2\rfloor}=\max \mu\left(x_{1}^{\lfloor r / 2\rfloor}\right)$. Usando a desigualdade (2.25), afirmamos que,

$$
\mu\left(B_{n}(j)\right)<C_{\rho} \inf _{1 \leq m<\lfloor n-j / 2\rfloor}\left(\rho^{m}+\beta_{m}\left(\frac{n-j}{2}\right)\right)+\tilde{\beta}(j-\lfloor r / 2\rfloor),
$$


e somando $j$ desde $\lfloor n / 2\rfloor+1$ até $\lfloor c n\rfloor$, obtemos

$$
\begin{aligned}
\sum_{j=\lfloor n / 2\rfloor+1}^{\lfloor c n\rfloor} \mu\left(B_{n}(j)\right) & \left.<C_{\rho} \sum_{j=\lfloor n / 2\rfloor+1}^{\lfloor c n\rfloor} \inf _{1 \leq m<\lfloor n-j / 2\rfloor}\left(\rho^{m}+\beta_{m}\left(\frac{n-j}{2}\right)\right)+\tilde{\beta}(j-\lfloor r / 2\rfloor)\right) \\
& \leq C_{\rho} \sum_{j=\lfloor n(1-c) / 2\rfloor}^{\lceil n / 4\rceil} \inf _{1 \leq m<j}\left(\rho^{m}+\beta_{m}(j)\right)+\sum_{j=\lfloor n / 2\rfloor+1}^{c n} \tilde{\beta}(j-\lfloor r / 2\rfloor) .
\end{aligned}
$$

Como $r<1 / 2, j-\lfloor r / 2\rfloor \geq j / 2$, portanto

$$
\begin{aligned}
\sum_{j=\lfloor n / 2\rfloor+1}^{\lfloor c n\rfloor} \tilde{\beta}(j-\lfloor r / 2\rfloor) & \leq \sum_{j=\lfloor n / 2\rfloor+1}^{\lfloor c n\rfloor} \tilde{\beta}(j / 2) \\
& \leq \sum_{j=\lfloor n / 4\rfloor}^{\lfloor c n\rfloor / 2} \tilde{\beta}(j) .
\end{aligned}
$$

Levando em conta que a função $\beta$ é decrescente, $\tilde{\beta}_{m}(j) \geq \tilde{\beta}(j)$ e que como $\lfloor n(1-c) / 2\rfloor \leq$ $\lfloor n / 4\rfloor$ porque $c>1 / 2$, a desigualdade (2.31) fica

$$
\sum_{j=\lfloor n / 2\rfloor+1}^{\lfloor c n\rfloor} \mu\left(B_{n}(j)\right)<2 C_{\rho} \sum_{j=\lfloor n(1-c) / 2\rfloor}^{\lfloor c n\rfloor / 2} \inf _{1 \leq m<j}\left(\rho^{m}+\beta_{m}(j)\right) .
$$

Somamos as expressoẽs (2.28) e (2.32), lembrando que $c>1 / 2$, obteremos

$$
\sum_{j=\lceil\lfloor c n\rfloor / 2\rceil}^{\lfloor c n\rfloor} \mu\left(B_{n}(j)\right)<C_{\rho}^{*} \sum_{j=\lfloor n(1-c) / 2\rfloor}^{\lfloor c n\rfloor} \inf _{1 \leq m<j}\left(\rho^{m}+\tilde{\beta}_{m}(j)\right) .
$$

Logo, segundo as inequações (2.26) e (2.33), é verdade que para qualquer constante $c$ entre zero e um,

$$
\sum_{j=\lceil\lfloor c n\rfloor / 2\rceil}^{\lfloor c n\rfloor} \mu\left(B_{n}(j)\right)<K_{c, \rho} \sum_{j=\left\lfloor c^{\prime} n\right\rfloor}^{\lfloor c n\rfloor} \inf _{1 \leq m<j}\left(\rho^{m}+\tilde{\beta}_{m}(j)\right),
$$

onde $K_{c, \rho}$ é uma constante que depende de $c$ e $\rho$ e $c^{\prime}=\min \{c / 2,(1-c) / 2\}$, acabando assim, a prova do lema.

Finalmente, vamos provar o teorema principal com ajuda dos lemas anteriores.

Demostração do Teorema 2.1

Note que pela definição dos conjuntos $R_{n}(j)$ e pelo processo ter gramática completa, temos 
que

$$
\begin{aligned}
\mu\left(S_{n} \geq k\right) & =\mu\left(\bigcup_{i=k}^{n-1} R_{n}(i)\right) \\
& =\mu\left(\bigcup_{i=k}^{\left\lfloor\frac{n}{4}\right\rfloor} R_{n}(i)\right)+\mu\left(\bigcup_{i=\left\lfloor\frac{n}{4}\right\rfloor+1}^{n-1} R_{n}(i) \backslash \bigcup_{j=k}^{\left\lfloor\frac{n}{4}\right\rfloor} R_{n}(j)\right) .
\end{aligned}
$$

Analisaremos o primeiro termo na equação (2.34)

$$
\begin{aligned}
\mu\left(\bigcup_{i=k}^{\left\lfloor\frac{n}{4}\right\rfloor} R_{n}(i)\right)= & \mu\left(R_{n}(k)\right)+\mu\left(R_{n}(k+1) \backslash R_{n}(k)\right)+\mu\left(R_{n}(k+2) \backslash R_{n}(k) \cup R_{n}(k+1)\right) \\
& +\cdots+\mu\left(R_{n}\left(\left\lfloor\frac{n}{4}\right\rfloor\right) \backslash \bigcup_{j=k}^{\left\lfloor\frac{n}{4}\right\rfloor-1} R_{n}(j)\right) \\
= & \mu\left(R_{n}(k)\right)+\sum_{i=k+1}^{\left\lfloor\frac{n}{4}\right\rfloor} \mu\left(R_{n}(i) \backslash \bigcup_{j=k}^{i-1} R_{n}(j)\right) .
\end{aligned}
$$

Vamos estudar cada termo da equação (2.35) separadamente.

Comecemos com o termo $\mu\left(R_{n}(k)\right)$. A idea é aplicar a propriedade $\beta$-mixing para limitar esta quantidade.

$$
\mu\left(R_{n}(k)\right)=\sum_{\omega \in R_{n}(k)} \mu(\omega) .
$$

Se $\omega \in R_{n}(k), \omega=x_{1}^{k} x_{k+1}^{n-k} x_{1}^{k}$. Removemos o bloco do meio, $x_{k+1}^{n-2 k}$, obtendo

$$
\mu(\omega) \leq \mu\left(x_{1}^{k}, x_{1}^{k}\right)
$$

$\log \mathrm{O}$

$$
\sum_{\omega \in R_{n}(k)} \mu(\omega) \leq \sum_{x_{1}^{k}} \mu\left(x_{1}^{k}, x_{1}^{k}\right) .
$$

Por outro lado, pela propriedade $\beta$-mixing que o processo nos fornece,

$$
\sum_{x_{1}^{k}}\left|\mu\left(x_{1}^{k}, x_{1}^{k}\right)-\mu^{2}\left(x_{1}^{k}\right)\right| \leq \tilde{\beta}(n-2 k) .
$$

Portanto,

$$
\begin{aligned}
\left|\mu\left(R_{n}(k)\right)-\sum_{x_{1}^{k}} \mu^{2}\left(x_{1}^{k}\right)\right| & =\left|\sum_{\omega \in R_{n}(k)} \mu(\omega)-\sum_{x_{1}^{k}} \mu^{2}\left(x_{1}^{k}\right)\right| \\
& \leq \sum_{x_{1}^{k}}\left|\mu\left(x_{1}^{k}, x_{1}^{k}\right)-\mu^{2}\left(x_{1}^{k}\right)\right| \\
& \leq \tilde{\beta}(n-2 k)
\end{aligned}
$$

Passamos agora ao somatório da equação (2.35). Se $\omega \in R_{n}(i)$, então $\omega=x_{1}^{i} x_{i+1}^{n-2 i} x_{1}^{i}$. O 
termo genérico do somatório é

$$
\begin{aligned}
\mu\left(R_{n}(i) \backslash \bigcup_{j=k}^{i-1} R_{n}(j)\right) & =\sum_{\omega \notin \bigcup_{j=k}^{i-1} R_{n}(j)} \mu(\omega) \\
& =\sum_{\omega: x_{1}^{i} \notin \bigcup_{j=k}^{i-1} R_{i}(j)} \mu(\omega) .
\end{aligned}
$$

Com o objetivo de usar a propriedade $\beta$ que o processo nos fornece, removemos o bloco $x_{i+1}^{n-2 i}$ de $\omega$ obtendo que

$$
\mu\left(x_{1}^{i} x_{i+1}^{n-2 i} x_{1}^{i}\right) \leq \mu\left(x_{1}^{i}, x_{1}^{i}\right) .
$$

Portanto,

$$
\sum_{x_{1}^{i} \notin \cup_{j=k}^{i-1} R_{i}(j)}\left|\mu\left(x_{1}^{i}, x_{1}^{i}\right)-\mu^{2}\left(x_{1}^{i}\right)\right| \leq \tilde{\beta}(n-2 i) .
$$

Isso nos permite afirmar que

$$
\begin{aligned}
\left|\mu\left(R_{n}(i) \backslash \bigcup_{j=k}^{i-1} R_{n}(j)\right)-\sum_{x_{1}^{i} \notin \cup_{j=k}^{i-1} R_{i}(j)} \mu^{2}\left(x_{1}^{i}\right)\right| & =\left|\sum_{\omega \notin \cup_{j=k}^{i-1} R_{i}(j)} \mu(\omega)-\sum_{x_{1}^{i} \notin \cup_{j=k}^{i-1} R_{i}(j)} \mu^{2}\left(x_{1}^{i}\right)\right| \\
& \leq \sum_{\substack{x_{1}^{i} \notin \cup_{j=k}^{i-1} R_{i}(j)\\
}}\left|\mu\left(x_{1}^{i}, x_{1}^{i}\right)-\mu^{2}\left(x_{1}^{i}\right)\right| \\
& \leq \tilde{\beta}(n-2 i)
\end{aligned}
$$

Somando nos dois lados da desigualdade anterior, desde $i=k+1$, até $\left\lfloor\frac{n}{4}\right\rfloor$, temos que,

$$
\begin{aligned}
\left|\sum_{i=k+1}^{\left\lfloor\frac{n}{4}\right\rfloor} \mu\left(R_{n}(i) \backslash \bigcup_{j=k}^{i-1} R_{n}(j)\right)-\sum_{i=k+1}^{\left\lfloor\frac{n}{4}\right\rfloor} \sum_{\omega \cup_{j=k}^{i-1} R_{i}(j)} \mu^{2}(\omega)\right| & \leq \sum_{i=k+1}^{\left\lfloor\frac{n}{4}\right\rfloor} \tilde{\beta}(n-2 i) \\
& \leq \sum_{i=\left\lceil\frac{n}{4}\right\rceil}^{\frac{n}{2}-(k+1)} \tilde{\beta}(2 i) .
\end{aligned}
$$

Analisaremos agora o segundo somando na equação (2.34). Observe que aplicando a relação de dualidade,

$$
\begin{aligned}
\mu\left(\bigcup_{i=\left\lfloor\frac{n}{4}\right\rfloor+1}^{n-1} R_{n}(i) \backslash \bigcup_{j=k}^{\left\lfloor\frac{n}{4}\right\rfloor} R_{n}(j)\right) & \leq \mu\left(\bigcup_{i=\left\lfloor\frac{n}{4}\right\rfloor+1}^{n-1} R_{n}(i)\right) \\
& \leq \mu\left(\bigcup_{i=1}^{\left\lfloor\frac{3 n}{4}\right\rfloor} B_{n}(i)\right)
\end{aligned}
$$


Substituindo $\mu\left(S_{n} \geq k\right)$ na seguinte expressão, temos que

$$
\begin{aligned}
& \left|\mu\left(S_{n} \geq k\right)-\mathbb{E}\left(\mu\left(X_{1}^{k}\right)\right)-a_{k}\right| \leq \\
& \quad\left|\mu\left(R_{n}(k)\right)+\sum_{i=k+1}^{\left\lfloor\frac{n}{4}\right\rfloor} \mu\left(R_{n}(i) \backslash \bigcup_{j=k}^{i-1} R_{n}(j)\right)+\mu\left(\bigcup_{i=1}^{\left\lfloor\frac{3 n}{4}\right\rfloor} B_{n}(i)\right)-\mathbb{E}\left(\mu\left(X_{1}^{k}\right)\right)-a_{k}\right| .
\end{aligned}
$$

Para facilitar a prova do teorema, denotemos por

$$
a_{k, n}=\sum_{i=k+1}^{\left\lfloor\frac{n}{4}\right\rfloor} \sum_{\omega \notin \cup \cup i=k} \mu^{2}(\omega)
$$

Somando e subtraindo o termo $a_{k, n}$ chegamos a

$$
\begin{aligned}
& \left|\mu\left(S_{n} \geq k\right)-\mathbb{E}\left(\mu\left(X_{1}^{k}\right)\right)-a_{k}\right| \leq \\
& \quad\left|\mu\left(R_{n}(k)\right)-\mathbb{E}\left(\mu\left(X_{1}^{k}\right)\right)\right|+\left|\sum_{i=k+1}^{\left\lfloor\frac{n}{4}\right\rfloor} \mu\left(R_{n}(i) \backslash \bigcup_{j=k}^{i-1} R_{n}(j)\right)-a_{k, n}\right|+\left|a_{k, n}-a_{k}\right|+\mu\left(\bigcup_{i=1}^{\left\lfloor\frac{3 n}{4}\right\rfloor} B_{n}(i)\right) .
\end{aligned}
$$

Agora, pelas equações (2.36) e (2.37), temos que

$$
\left|\mu\left(R_{n}(i)\right)-\mathbb{E}\left(\mu\left(X_{1}^{k}\right)\right)\right|+\left|\sum_{i=k+1}^{\left\lfloor\frac{n}{4}\right\rfloor} \mu\left(R_{n}(i) \backslash \bigcup_{j=k}^{i-1} R_{n}(j)\right)-a_{k, n}\right| \leq \sum_{i=\left\lfloor\frac{n}{4}\right\rfloor}^{\left\lceil\frac{n}{2}\right\rceil-k} \tilde{\beta}(2 i) .
$$

A equação (2.16) garante que

$$
\begin{aligned}
\left|a_{k, n}-a_{k}\right| & =\sum_{i=\left\lfloor\frac{n}{4}\right\rfloor+1}^{\infty} \sum_{\omega \notin \cup_{j=k}^{i-1} R_{i}(j)} \mu^{2}(\omega) \\
& \leq \sum_{i=\left\lfloor\frac{n}{4}\right\rfloor+1}^{\infty} \mathbb{E}\left(\mu\left(X_{1}^{i}\right)\right) \\
& \leq \sum_{i=\left\lfloor\frac{n}{4}\right\rfloor+1}^{\infty} \inf _{1 \leq m<i}\left(\rho^{m+1}+\frac{\beta_{m}(i)}{2(1-\rho)}\right) .
\end{aligned}
$$

E, pelo Lema (2.8), $\operatorname{com} c=3 / 4$ temos que

$$
\mu\left(\bigcup_{i=1}^{\left\lfloor\frac{3 n}{4}\right\rfloor} B_{n}(i)\right) \leq K_{\rho}^{\prime} \sum_{j=\left\lfloor\frac{n}{8}\right\rfloor}^{\left\lfloor\frac{3 n}{4}\right\rfloor} \inf _{1 \leq m<j}\left(\rho^{m}+\tilde{\beta}_{m}(j)\right) .
$$


Somando as equações (2.41) e (2.42), lembrando que $\beta_{m}(i) \leq \tilde{\beta}_{m}(i)$, obtemos que

$$
\sum_{i=\left\lfloor\frac{n}{4}\right\rfloor+1}^{\infty} \inf _{1 \leq m<i}\left(\rho^{m+1}+\frac{\beta_{m}(i)}{2(1-\rho)}\right)+K_{\rho}^{\prime} \sum_{j=\left\lfloor\frac{n}{8}\right\rfloor}^{\left\lfloor\frac{3 n}{4}\right\rfloor} \inf _{1 \leq m<j}\left(\rho^{m}+\tilde{\beta}_{m}(j)\right) \leq C_{\rho}^{\prime} \sum_{i=\left\lfloor\frac{n}{8}\right\rfloor}^{\infty} \inf _{1 \leq m<i}\left(\rho^{m}+\tilde{\beta}_{m}(i)\right) .
$$

Substituindo as inequações (2.40) e (2.43), na desigualdade (2.39), chegamos a

$$
\left|\mu\left(S_{n} \geq k\right)-\mathbb{E}\left(\mu\left(X_{1}^{k}\right)\right)-a_{k}\right| \leq \sum_{i=\left\lfloor\frac{n}{4}\right\rfloor}^{\left\lceil\frac{n}{2}\right\rceil-k} \tilde{\beta}(2 i)+C_{\rho}^{\prime} \sum_{i=\left\lfloor\frac{n}{8}\right\rfloor}^{\infty} \inf _{1 \leq m<i}\left(\rho^{m}+\tilde{\beta}_{m}(i)\right) .
$$

Lembremos que a nossa função $\beta$ é decrescente, e portanto, $\tilde{\beta}_{m}(i) \geq \tilde{\beta}(i) \geq \tilde{\beta}(2 i)$, chegando assim ao resultado procurado na primeira parte do teorema,

$$
\left|\mu\left(S_{n} \geq k\right)-\mathbb{E}\left(\mu\left(X_{1}^{k}\right)\right)-a_{k}\right| \leq K_{\rho} \sum_{i=\left\lfloor\frac{n}{8}\right\rfloor}^{\infty} \inf _{1 \leq m<i}\left(\rho^{m}+\tilde{\beta}_{m}(i)\right)
$$

onde $K_{\rho}$ é uma contante que depende de $\rho$.

Vamos provar agora a convergência que será provada em duas partes. Primeiro, a convergência do somatório com termos $\rho^{m}$ e depois o somatório com termos $\beta_{m}(i)$. Para isso, tomemos $m=\log _{\rho}(1 / f(i))$, onde $f(i)=i(\ln i)^{1+\epsilon}$ para algum $\epsilon$ entre 0 e 1 . Então,

$$
\begin{aligned}
\sum_{i=\left\lfloor\frac{n}{8}\right\rfloor}^{\infty} \inf _{1 \leq m<i}\left(\rho^{m}+\tilde{\beta}_{m}(i)\right) & \leq \sum_{i=\left\lfloor\frac{n}{8}\right\rfloor}^{\infty}\left(\rho^{\log _{\rho}(1 / f(i))}+\tilde{\beta}_{\log _{\rho}(1 / f(i))}(i)\right) \\
& =\sum_{i=\left\lfloor\frac{n}{8}\right\rfloor}^{\infty}\left(\frac{1}{f(i)}+\tilde{\beta}\left(\left\lfloor\frac{i}{\log _{\rho}(1 / f(i))}\right\rfloor-1\right)\right) \\
& =\sum_{i=\left\lfloor\frac{n}{8}\right\rfloor}^{\infty} \frac{1}{f(i)}+\sum_{i=\left\lfloor\frac{n}{8}\right\rfloor}^{\infty} \tilde{\beta}\left(\left\lfloor\frac{i}{\log _{\rho}(1 / f(i))}\right\rfloor-1\right) .
\end{aligned}
$$

Queremos encontrar o limite da equação anterior quando $n$ diverge. É claro que o limite do primeiro somatório de termos $1 / f(i)$ tem limite zero porque esta série é somável. Assim, voltaremos nossa atenção à soma com termos $\beta$. O limite que nós procuramos, é igual ao limite da expressão $\sum_{i=\left\lfloor\frac{n}{8}\right\rfloor}^{\infty} \tilde{\beta}\left(\left\lfloor i \rho_{0} / \ln i\right\rfloor\right)$, onde $\rho_{0}=-\ln \rho / 2$. Note que $\rho_{0}>0$ porque $\ln \rho<0$. Vamos provar que este limite é zero. Comecemos afirmando que

$$
\frac{i}{\log _{\rho}(1 / f(i))}=\frac{i \ln \rho}{\ln (1 / f(i))}=\frac{-i \ln \rho}{\ln f(i)}
$$

Note que $\ln f(i)=\ln i+(1+\epsilon) \ln (\ln i)$. Além disso, $\ln i<i$ para todo $i$ e como $\epsilon<1$, então

$$
\begin{aligned}
& \ln f(i)<2 \ln i . \\
& \frac{i}{\ln f(i)}>\frac{i}{2 \ln i} .
\end{aligned}
$$


Nosso objetivo agora será contar, para $i$ fixo, o número de inteiros $h$ tais que

$$
\frac{h}{\ln h} \in\left[\left\lfloor\frac{i}{\ln i}\right\rfloor,\left\lfloor\frac{i}{\ln i}\right\rfloor+1\right) .
$$

Para contar tais $h$, vamos estudar o incremento $(i+1) / \ln (i+1)-i / \ln i$. Para $i$ suficientemente grande, temos que

$$
\frac{i+1}{\ln (i+1)}-\frac{i}{\ln i} \geq \frac{1}{2 \ln (i+1)}
$$

A quantidade de $h$ que satisfaz (2.46) é menor ou igual à quantidade de $h$ tal que

$$
\left\lfloor\frac{i}{\ln i}\right\rfloor+\frac{h}{\ln (i+1)} \in\left[\left\lfloor\frac{i}{\ln i}\right\rfloor,\left\lfloor\frac{i}{\ln i}\right\rfloor+1\right) .
$$

Isto é porque no mesmo intervalo $[\lfloor i / \ln i\rfloor,\lfloor i / \ln i\rfloor+1)$ estamos contando mais pontos. Da expressão (2.47) obtemos que

$$
h<\frac{2}{\rho_{0}} \ln (i+1) .
$$

Logo, como $\beta$ é uma função decrescente,

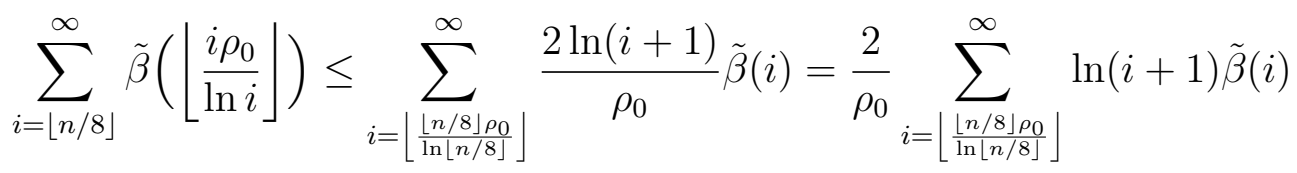

E como por hipótese ln $i \beta(i)$ é somável, obtemos o limite zero que procurávamos, e com isso termina nossa prova.

\section{Demostração da Proposição 2.3}

Para provar esta proposição, nós nos baseamos no fato de que o termo $a_{k}=\lim _{n \rightarrow \infty} a_{k, n}$ para $a_{k, n}$ como foi definido em (2.38). Por isso, vamos nos concentrar nessa última expressão. É claro que

$$
a_{k, n}=\sum_{i=k+1}^{\left\lfloor\frac{n}{4}\right\rfloor} \mu\left(R_{n}(i) \backslash \bigcup_{j=k}^{i-1} R_{n}(j)\right)=\sum_{i=k+1}^{\left\lfloor\frac{n}{4}\right\rfloor} \mu\left(R_{n}(i)\right)-\sum_{i=k+1}^{\left\lfloor\frac{n}{4}\right\rfloor} \mu\left(R_{n}(i) \cap \bigcup_{j=k}^{i-1} R_{n}(j)\right)
$$

Observe que $\mu\left(R_{n}(i) \cap \bigcup_{j=k}^{i-1} R_{n}(j)\right) \geq \mu\left(R_{n}(i) \cap R_{n}(k)\right)$, logo,

$$
\sum_{i=k+1}^{\left\lfloor\frac{n}{4}\right\rfloor} \mu\left(R_{n}(i) \backslash \bigcup_{j=k}^{i-1} R_{n}(j)\right) \leq \sum_{i=k+1}^{\left\lfloor\frac{n}{4}\right\rfloor} \mu\left(R_{n}(i)\right)-\sum_{i=k+1}^{\left\lfloor\frac{n}{4}\right\rfloor} \mu\left(R_{n}(i) \cap R_{n}(k)\right)
$$

Se $\omega \in R_{n}(i), \omega=x_{1}^{i} x_{i+1}^{n-2 i} x_{1}^{i}$. Removendo o bloco $x_{i+1}^{n-2 i}$, obtemos que

$$
\mu(\omega) \leq \mu\left(x_{1}^{i}, x_{1}^{i}\right)
$$


e, pela propriedade $\beta$-mixing do processo

$$
\begin{aligned}
\left|\mu\left(R_{n}(i)\right)-\mathbb{E}\left(\mu\left(X_{1}^{i}\right)\right)\right| & =\left|\sum_{\omega \in R_{n}(i)} \mu(\omega)-\sum_{x_{1}^{i}} \mu^{2}\left(x_{1}^{i}\right)\right| \\
& \leq \sum_{x_{1}^{i}}\left|\mu\left(x_{1}^{i}, x_{1}^{i}\right)-\mu^{2}\left(x_{1}^{i}\right)\right| \\
& \leq \tilde{\beta}(n-2 i)
\end{aligned}
$$

Logo,

$$
\mu\left(R_{n}(i)\right) \leq \mathbb{E}\left(\mu\left(X_{1}^{i}\right)\right)+\tilde{\beta}(n-2 i) .
$$

Por outro lado, temos que

$$
\begin{aligned}
\mu\left(R_{n}(i) \cap R_{n}(k)\right) & =\sum_{\omega \in R_{n}(i) \cap R_{n}(k)} \mu(\omega) \\
& =\sum_{\omega \in R_{n}(k)} \mu\left(x_{1}^{i} x_{i+1}^{n-2 i} x_{1}^{i}\right) .
\end{aligned}
$$

Removendo o bloco $x_{i+1}^{n-2 i}$,

$$
\mu\left(R_{n}(i) \cap R_{n}(k)\right)<\sum_{x_{1}^{i} \in R_{i}(k)} \mu\left(x_{1}^{i}, x_{1}^{i}\right) .
$$

Aplicando a propriedade $\beta$-mixing do processo chegamos a

$$
\begin{aligned}
\left|\mu\left(R_{n}(i) \cap R_{n}(k)\right)-\sum_{x_{1}^{i} \in R_{i}(k)} \mu^{2}\left(x_{1}^{i}\right)\right| & =\left|\sum_{x_{1}^{n} \in R_{n}(i) \cap R_{n}(k)} \mu\left(x_{1}^{n}\right)-\sum_{x_{1}^{i} \in R_{i}(k)} \mu^{2}\left(x_{1}^{i}\right)\right| \\
& \leq \sum_{x_{1}^{i} \in R_{i}(k)}\left|\mu\left(x_{1}^{i}, x_{1}^{i}\right)-\mu^{2}\left(x_{1}^{i}\right)\right| \\
& \leq \tilde{\beta}(n-2 i) .
\end{aligned}
$$

Portanto,

$$
\mu\left(R_{n}(i) \cap R_{n}(k)\right) \geq \sum_{\omega \in R_{i}(k)} \mu^{2}(\omega)-\tilde{\beta}(n-2 i) .
$$

Se somarmos em $i$ desde $k+1$ até $\lfloor n / 4\rfloor$ e se levarmos em conta as equações (2.50) e (2.51), na equação (2.49), teremos que

$$
\begin{aligned}
\sum_{i=k+1}^{\left\lfloor\frac{n}{4}\right\rfloor} \mu\left(R_{n}(i) \backslash \bigcup_{j=k}^{i-1} R_{n}(j)\right) & =\sum_{i=k+1}^{\left\lfloor\frac{n}{4}\right\rfloor} \mu\left(R_{n}(i)\right)-\sum_{i=k+1}^{\left\lfloor\frac{n}{4}\right\rfloor} \mu\left(R_{n}(i) \cap R_{n}(k)\right) \\
& \leq \sum_{i=k+1}^{\left\lfloor\frac{n}{4}\right\rfloor} \mathbb{E}\left(\mu\left(X_{1}^{i}\right)\right)-\sum_{i=k+1}^{\left\lfloor\frac{n}{4}\right\rfloor} \sum_{\omega \in R_{i}(k)} \mu^{2}(\omega)+2 \sum_{i=k+1}^{\left\lfloor\frac{n}{4}\right\rfloor} \tilde{\beta}(n-2 i) \\
& =\sum_{i=k+1}^{\left\lfloor\frac{n}{4}\right\rfloor} \mathbb{E}\left(\mu\left(X_{1}^{i}\right)\right)-\sum_{i=k+1}^{\left\lfloor\frac{n}{4}\right\rfloor} \sum_{\omega \in R_{i}(k)} \mu^{2}(\omega)+2 \sum_{i=\left\lceil\frac{n}{4}\right\rceil}^{\frac{n}{2}-(k+1)} \tilde{\beta}(2 i) .
\end{aligned}
$$

Como ln $i \beta(i)$ é somavel por hipótese, $\beta(i)$ é somável também, ficando assim provada a nossa 
proposição.

Demostração do Proposição 2.4

Da mesma maneira que se começou a proposição anterior, temos que

$$
a_{k, n}=\sum_{i=k+1}^{\left\lfloor\frac{n}{4}\right\rfloor} \mu\left(R_{n}(i) \backslash \bigcup_{j=k}^{i-1} R_{n}(j)\right) \geq \sum_{i=k+1}^{\left\lfloor\frac{n}{4}\right\rfloor} \mu\left(R_{n}(i)\right)-\sum_{i=k+1}^{\left\lfloor\frac{n}{4}\right\rfloor} \sum_{j=k}^{i-1} \mu\left(R_{n}(i) \cap \mu\left(R_{n}(j)\right) .\right.
$$

Pelas equações $(2.50)$ e (2.51),

$$
\sum_{i=k+1}^{\left\lfloor\frac{n}{4}\right\rfloor} \mu\left(R_{n}(i) \backslash \bigcup_{j=k}^{i-1} R_{n}(j)\right) \geq \sum_{i=k+1}^{\left\lfloor\frac{n}{4}\right\rfloor} \mathbb{E}\left(\mu\left(X_{1}^{i}\right)\right)-\sum_{i=k+1}^{\left\lfloor\frac{n}{4}\right\rfloor} \sum_{j=k}^{i-1} \sum_{\omega \in R_{i}(k)} \mu^{2}(\omega)+\sum_{i=\left\lfloor\frac{n}{4}\right\rfloor}^{\left\lfloor\frac{n}{2}\right\rfloor-k\left\lfloor\frac{n}{2}\right\rfloor-k} \tilde{j} \tilde{\beta}(2 i) .
$$

Vamos desenvolver o somatório $\sum_{i=\left\lfloor\frac{n}{4}\right\rfloor}^{\left\lfloor\frac{n}{2}\right\rfloor-k} \sum_{j=i}^{\left\lfloor\frac{n}{2}\right\rfloor-k} \tilde{\beta}(2 i)$.

$$
\begin{aligned}
\sum_{i=\left\lfloor\frac{n}{4}\right\rfloor}^{\left\lfloor\frac{n}{2}\right\rfloor-k\left\lfloor\frac{n}{2}\right\rfloor-k} \tilde{j} \tilde{\beta}(2 i) & =\sum_{j=\left\lfloor\frac{n}{4}\right\rfloor}^{\left\lfloor\frac{n}{2}\right\rfloor-k} \tilde{\beta}(2 i)+\sum_{j=\left\lfloor\frac{n}{4}\right\rfloor+1}^{\left\lfloor\frac{n}{2}\right\rfloor-k} \tilde{\beta}(2 i)+\cdots+\tilde{\beta}\left(2\left(\left\lfloor\frac{n}{2}\right\rfloor-k\right)\right) \\
& =\sum_{i=\left\lfloor\frac{n}{4}\right\rfloor}^{\left\lfloor\frac{n}{2}\right\rfloor-k}\left(i-\left\lfloor\frac{n}{4}\right\rfloor\right) \tilde{\beta}(2 i) .
\end{aligned}
$$

Usando a hipótese da somabilidade de $i \ln i \beta(i)$ concluímos nossa proposição.

No seguinte exemplo, mostramos um processo que satisfaz as condições do Teorema 2.1

\subsubsection{Exemplos}

\subsubsection{Exemplo 1}

Este exemplo mostra um processo que cumpre as condições do Teorema 2.1.

Considere uma cadeia de Markov $\left\{Y_{n}\right\}_{n \in \mathbb{N}}$, com espaço de estados $\{0,1,2, \ldots\}$, e os componentes da matriz de transição $Q_{Y}$ dadas por:

$$
\begin{aligned}
Q_{Y}(y, 0) & =1-q_{y} \\
Q_{Y}(y, y+1) & =q_{y},
\end{aligned}
$$

para $0<q_{y}<1$ e 0 nos outros casos. A figura seguinte mostra graficamente tal processo.

Esse processo foi objeto de estudo de Fernández, Ferrari e Galves em [12]. A cadeia de Markov define um processo de renovação. Sejam $Y_{0}=0$,

$$
\begin{gathered}
T_{1}=\inf \left\{n \geq 1: Y_{n}=0\right\} \quad \text { e } \\
T_{m}=\inf \left\{n \geq T_{m-1}: Y_{n}=0\right\}, \quad \text { para todo } m \geq 2 .
\end{gathered}
$$

As propriedades de Markov garantem que as variáveis aleatórias $T_{1}, T_{2}-T_{1}, \ldots, T_{m}-$ 


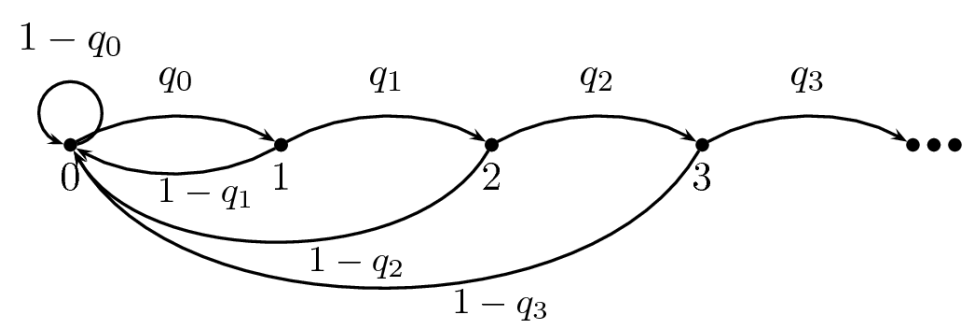

Figura 2.1: Cadeia $\left\{Y_{n}\right\}_{n \geq 0}$

$T_{m-1}, m \geq 1$ são independentes a cada duas e, que se denotarmos por $Z_{m}=T_{m}-T_{m-1}, m \geq$ 2 , as variáveis $\left\{Z_{m}\right\}_{m \geq 2}$, são identicamente distribuídas. Vamos calcular a distribuição de $Z_{m}$.

$$
\begin{aligned}
\mu\left(Z_{m}=k\right) & =\sum_{i=1}^{\infty} \mu\left(T_{m}=k+i \mid T_{m-1}=i\right) \mu\left(T_{m-1}=i\right) \\
& =\sum_{i=1}^{\infty} q_{0} q_{1} \ldots q_{k-2}\left(1-q_{k-1}\right) \mu\left(T_{m-1}=i\right) \\
& =\left(1-q_{k-1}\right) \prod_{i=0}^{k-2} q_{j} \quad \text { para todo } m \geq 2 .
\end{aligned}
$$

Agora vejamos que o valor esperado de $Z_{m}$ não depende de $m$. Se $\max _{j} q_{j}$ existe $\max _{j} q_{j}<1$, pode-se provar que, de fato, $\mathbb{E}\left(Z_{m}\right)$ não depende de $m$. Por isso, vamos considerar o seguinte caso, um pouco mais interessante, pois $\lim _{j \rightarrow \infty} q_{j}=1$.

Sejam $q_{0}=1$ e $q_{j}=\left(\frac{j}{j+1}\right)^{s}$, onde $s \in \mathbb{R}^{+}$. Nesse caso, temos que para $m \geq 2$

$$
\begin{aligned}
\mathbb{E}\left(Z_{m}\right) & =\sum_{k=2}^{\infty} k\left(1-q_{k-1}\right) \prod_{i=0}^{k-2} q_{j} \\
& =\sum_{k=2}^{\infty} k\left(1-\frac{k-1}{k}\right)\left(\frac{1}{k-1}\right)^{s} \\
& =\sum_{k=2}^{\infty}\left(\frac{1}{k-1}\right)^{s}
\end{aligned}
$$

A última expressão converge quando $s>1$.

Vamos definir o processo que indica quando o $\left\{Y_{n}\right\}_{n \geq 0}$ é igual a zero, ie. definimos o processo $\left\{X_{n}\right\}_{n \geq 0}$ no alfabeto $\{0,1\}$ tal que $X_{n}=1$ quando $Y_{n}=0$, e $X_{n}=0$ quando $Y_{n}$ assume um valor diferente de zero. Isto é, $X_{n}=G\left(y_{n}\right)$, com

$$
\begin{gathered}
G:\{0,1, \ldots\} \rightarrow\{0,1\} \\
x \mapsto \begin{cases}1 & \text { se } y=0, \\
0 & \text { se } y>0 .\end{cases}
\end{gathered}
$$

A distribuição de $\left\{X_{n}\right\}_{n \geq 0}$ é unicamente determinada pela distribuição de $\left\{Z_{n}\right\}_{n \geq 0}$. Para concluir se a sequência $\left\{X_{n}\right\}_{n \geq 0}$ é $\beta$-mixing com coeficientes somáveis, vamos a aplicar o seguinte teorema extraído de [10]: 
Teorema 2.9. Seja $P$ uma distribuição de probabilidade sobre os números naturais e sejam $Z_{1}^{\prime}, Z_{2}^{\prime}, \ldots$ variáveis aleatórias independentes com distribuição comum $P$ tal que, o máximo divisor comum, $\operatorname{mdc}\{i: P(i)>0\}=1$. Suponha que o valor esperado de $Z_{m}^{\prime}$ existe para todo $m$. Considere também o processo,

$$
X_{n}^{\prime}= \begin{cases}1 & \text { se } n=Z_{1}^{\prime}+Z_{2}^{\prime}+\cdots+Z_{t}^{\prime} \text { para algum } t \\ 0 & \text { caso contrário, }\end{cases}
$$

então, $P$ tem $r$-ésimo momento finito, com $r>1$ se e somente se $\sum_{n \geq 1} n^{r-2} \beta(n)<\infty$ para o processo de renovação $\left\{X_{n}^{\prime}\right\}_{n \geq 0}$.

Para que o teorema anterior seja válido no nosso exemplo, falta verificar que se cumprem as hipóteses.

$$
\left\{i: \mu\left(Z_{m}=i\right)>0\right\}=\left\{i: \frac{1}{i(i-1)^{s}}>0\right\},
$$

$\operatorname{logo} \operatorname{mdc}\left\{i: \mu\left(Z_{m}=i\right)>0\right\}=1$. Agora, vamos provar que a distribuição do processo de renovação tem algum momento finito.

$$
\mathbb{E}\left(Z_{m}^{r}\right)=\sum_{k=2}^{\infty} k^{r}\left(1-q_{k-1}\right) \prod_{i=0}^{k-2} q_{j} .
$$

No caso onde $\max _{j} q_{j}<1$, pode-se provar que existem todos os momentos finitos de ordem $r \geq 2$. Mas no nosso caso, quando $q_{j}=\left(\frac{j}{j+1}\right)^{s}$ acontece o seguinte:

$$
\begin{aligned}
\mathbb{E}\left(Z_{m}^{r}\right) & =\sum_{k=2}^{\infty} k^{r}\left(1-q_{k-1}\right) \prod_{i=0}^{k-2} q_{j} \\
& =\sum_{k=2}^{\infty} k^{r}\left(1-\frac{k-1}{k}\right)\left(\frac{1}{k-1}\right)^{s} \\
& =\sum_{k=2}^{\infty} \frac{k^{r-1}}{(k-1)^{s}},
\end{aligned}
$$

onde a última linha converge para $r<s$. Podemos concluir que o processo de renavação $\left\{X_{n}\right\}_{n \in \mathbb{N}}$ é $\beta$-mixing com coeficientes $\ln j \beta(j)$ somáveis se $r$ for maior que dois, para o qual seria necessário que $s$ seja maior do que três. Note que o processo $\left\{X_{n}\right\}_{n \in \mathbb{N}}$ tem gramática completa, pois qualquer sequência de zeros e uns tem probabilidade positiva de acontecer, então vale o Teorema 2.1 e, portanto, existe a distribuição limite para a variável aleatória $S_{n}$.

No próximo exemplo, vamos ver um processo ergódico não $\beta$-mixing, com entropia positiva para o qual não vale o Teorema 2.1.

\subsubsection{Exemplo 2}

Este exemplo mostra um processo que não é $\beta$-mixing com gramática completa e que não cumpre o Teorema 2.1.

Seja $\left\{X_{n}\right\}_{n \geq 0}$ um processo definido sobre o alfabeto $\{1,2,3,4\}$. Considere as etiquetas $A$, para indicar quando o processo pertence a $\{1,2\}$ e $B$ para indicar quando o processo pertence a $\{3,4\}$. Lança-se uma moeda não tendenciosa para indicar se $X_{0} \in A$ ou $X_{0} \in B$ 
e considere $\epsilon>0$. Se $X_{0} \in A$, para $n \geq 1$, temos

$$
X_{2 n} \in \begin{cases}A & \text { com probabilidade } 1-\epsilon \\ B & \text { com probabilidade } \epsilon\end{cases}
$$

e

$$
X_{2 n+1} \in\left\{\begin{array}{l}
B \text { com probabilidade } 1-\epsilon, \\
A \text { com probabilidade } \epsilon .
\end{array}\right.
$$

Se $X_{0} \in B$, ocorrerá o contrário. Nos $n$ pares, $X_{n}$ terá probabilidade $1-\epsilon$ de pertencer a $B$ e probabilidade $\epsilon$ de pertencer a $A$. Para $n$ ímpar, $X_{n}$ terá probabilidade $1-\epsilon$ de pertencer a $A$ e probabilidade $\epsilon$ de pertencer a $B$. Desta maneira, o processo gerado tem gramática completa.

O processo descrito anteriormente não perde memória e, por isso, ele não é $\beta$-mixing. Para ilustrar isto, considere a seguinte situação para qualquer $j \geq 1$ e $n$ fixo.

$$
\mu\left(X_{n}=1, X_{n+j}=1\right)=\frac{1}{2} \mu\left(X_{n}=1, X_{n+j}=1 \mid X_{0} \in A\right)+\frac{1}{2} \mu\left(X_{n}=1, X_{n+j}=1 \mid X_{0} \in B\right),
$$

o qual implica que

$$
\mu\left(X_{n}=1, X_{j+n}=1\right)= \begin{cases}\frac{1}{8}\left(\epsilon^{2}+(1-\epsilon)^{2}\right) & \text { se } j \text { for par, ou } \\ \frac{1}{4} \epsilon(1-\epsilon) & \text { se } n \text { for ímpar. }\end{cases}
$$

Agora, sem importar se $n$ é par ou ímpar,

$$
\begin{aligned}
\mu\left(X_{n}=1\right) & =\frac{1}{2} \mu\left(X_{n}=1 \mid X_{0} \in A\right)+\frac{1}{2} \mu\left(X_{n}=1 \mid X_{0} \in B\right) \\
& =\frac{1}{4} .
\end{aligned}
$$

Logo, o nosso processo não é $\beta$-mixing. Por outro lado, a função de sobreposição $S_{n}$, não converge em distribuição. Intuitivamente isto é porque a função $S_{n}$, toma valores alternados dependendo se $n$ for par ou não. Comecemos analizando $\mu\left(S_{2 n}=2 k\right)$ para $k<n$ usando $\omega_{j}$ para denotar uma palavra de tamanho $j$.

$$
\begin{aligned}
\mu\left(S_{2 n}=2 k\right) & \leq \mu\left(R_{2 n}(2 k)\right) \\
& \leq \frac{1}{2} \mu\left(X_{1}^{2 k}=\omega_{2 k}, X_{2 n-2 k+1}^{2 n}=\omega_{2 k} \mid X_{0} \in A\right)+\frac{1}{2} \mu\left(X_{1}^{2 k}=\omega_{2 k}, X_{2 n-2 k+1}^{2 n}=\omega_{2 k} \mid X_{0} \in B\right) \\
& =\left(\epsilon^{2}+(1-\epsilon)^{2}\right)^{2 k} .
\end{aligned}
$$

Portanto, $\mu\left(S_{2 n}=2 k+1\right)>1-\left(\epsilon^{2}+(1-\epsilon)^{2}\right)^{2 k}$. Agora,

$$
\begin{aligned}
\mu\left(S_{2 n+1}=2 k+1\right) \leq & \mu\left(R_{2 n+1}(2 k+1)\right) \\
\leq & \frac{1}{2} \mu\left(X_{1}^{2 k+1}=\omega_{2 k+1}, X_{2 n+1-2 k}^{2 n+1}=\omega_{2 k+1} \mid X_{0} \in A\right)+ \\
& \frac{1}{2} \mu\left(X_{1}^{2 k+1}=\omega_{2 k+1}, X_{2 n+1-2 k}^{2 n+1}=\omega_{2 k+1} \mid X_{0} \in B\right) \\
& =\left(\epsilon^{2}+(1-\epsilon)^{2}\right)^{2 k+1} .
\end{aligned}
$$

Isto implica que o comportamento da $\mu\left(S_{n}=k\right)$ se intercala dependendo do valor de $n$ par ou não, o que nós leva a afirmar que a variável $S_{n}$ não converge em distribuição. 


\subsubsection{Exemplo 3}

Nesta subseção vamos mostrar, usando simulação, dois exemplos onde a probabilidade de $S_{n}=-1$ converge. Primeiro consideremos a cadeia de Markov do Exemplo 1.11 a qual não tem gramática completa mas é $\beta$-mixing. Esta cadeia assume valores no conjunto $\chi=$ $\{0,1,2\}$ e tem as seguintes probabilidades de transição:

$$
P=\frac{0}{1} \begin{array}{ccc}
0 & 1 & 2 \\
2
\end{array}\left(\begin{array}{ccc}
1-q_{0} & q_{0} & 0 \\
1-q_{1} & 0 & q_{1} \\
1-q_{2} & 0 & q_{2}
\end{array}\right)
$$

onde $0<q_{i}<1$, para $i=0,1,2$.

Note que $S_{n}$ não pode ser menor que -1, pois basta, no máximo, um passo só para que uma palavra que não tenha sobreposição comece de novo. Para esta primeira simulação, tomamos $q_{0}=0.2, q_{1}=0.3, q_{2}=0.4$ e depois $q_{0}=0.5, q_{1}=0.7, q_{2}=0.9$. Em ambos casos calculamos $\mu\left(S_{n}=-1\right)$ para $n$ desde 2 até 15 . Os gráficos resultantes mostram a convergência desta probabilidade.

\section{Probabilidade de $\mathrm{Sn}=-1$}

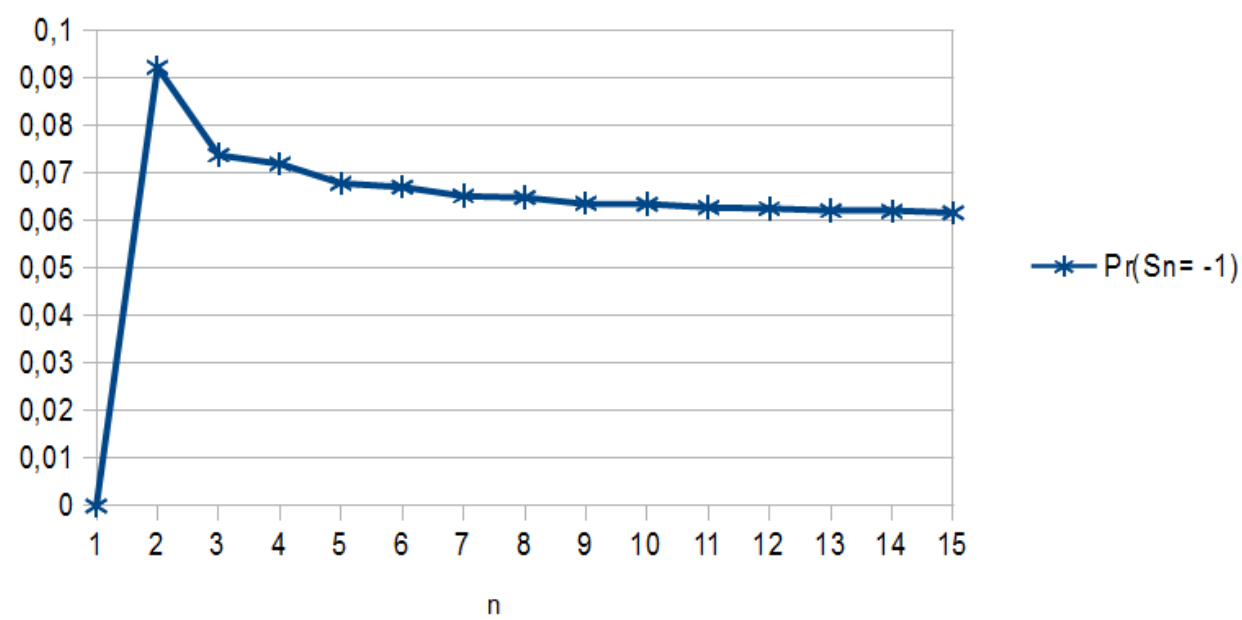

Figura 2.2: Convergência 1 de $\mu\left(S_{n}=-1\right)$. 


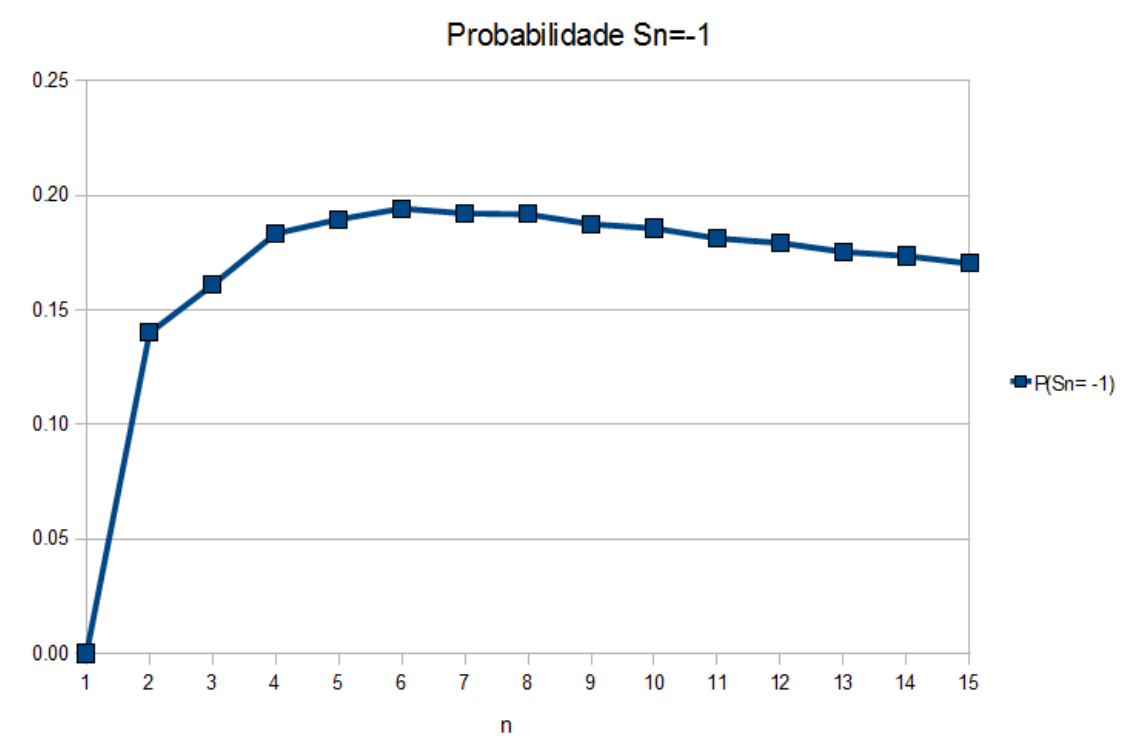

Figura 2.3: Convergência 2 de $\mu\left(S_{n}=-1\right)$.

No segundo exemplo, considere $\left\{X_{n}\right\}_{n \geq 0}$ um processo estocástico assumindo valores no alfabeto $\chi=\{0,1\}$. Lança-se uma moeda não tendenciosa para determinar se $X_{0}=1 \mathrm{ou}$ $X_{0}=0$, e para $n \geq 1$, as probabilidades de transição são dadas da seguinte maneira:

$$
\mu\left(X_{n}=1 \mid X_{n-1}=1\right)=\mu\left(X_{n}=0 \mid X_{n-1}=1\right)=\frac{1}{2} .
$$

Se $X_{n-1}=0$ então, devemos contar o número de zeros consecutivos no passado até o primeiro 1. Suponhamos que esse primeiro 1 no passado é encontrado em $l_{n}$, ou seja, $X_{l_{n}}=1$. Além disso, $X_{l_{n}+1}^{n-1}=0_{l_{n}+1}^{n-1}$ onde $0_{l_{n}+1}^{n-1}$ representa um bloco de zeros de tamanho $n-l_{n}-1$. Assim,

$$
\mu\left(X_{n}=1 \mid X_{l_{n}+1}^{n-1}=0_{l_{n}+1}^{n-1}\right)=\mu\left(X_{n}=0 \mid X_{l_{n}+1}^{n-1}=0_{l_{n}+1}^{n-1}\right)=\frac{1}{2},
$$

se $n-l_{n}$ for ímpar. Com esta mesma notação em mente, se $n-l_{n}$ for par,

$$
\mu\left(X_{n}=1 \mid X_{l_{n}-1}^{n-1}=0_{l_{n}-1}^{n-1}\right)=1 .
$$

Informalmente falando, se $X_{n-1}=1$ então $X_{n}=0$ ou $X_{n}=1$ com probabilidade igual a $1 / 2$ independentemente do que aconteceu no processo antes do tempo $n-1$. Se $X_{n-1}=0$, então devemos contar o número de zeros consecutivos que antecederam o tempo $n-1$ até achar o primeiro 1. Se o número total de zeros ( $X_{n-1}$ incluso) for ímpar, $X_{n}=1$ ou $X_{n}=0$ com probabilidade igual a $1 / 2$. Agora se esse número for par, o próximo estado do processo, em $n$, é obrigatoriamente 0. Isto implica que o processo não apresenta blocos de zeros de tamanho par com probabilidade positiva, portanto, o processo não tem gramática completa.

A pergunta que nós fazemos é se $\mu\left(S_{n}=-1\right)$ converge quando não sabemos se este processo é $\beta$-mixing. Para pesquisar a resposta, foram simuladas palavras de um processo com estas características. Os tamanhos das palavras variavam entre 2 e 20 e foi calculada para cada uma a probabilidade de $S_{n}=-1$. Note que $S_{n}$ não pode ser menor que -1 , pois basta no máximo, um passo só para que uma palavra que não tenha sobreposição comece de novo. Os resultados são representados no seguinte gráfico: 


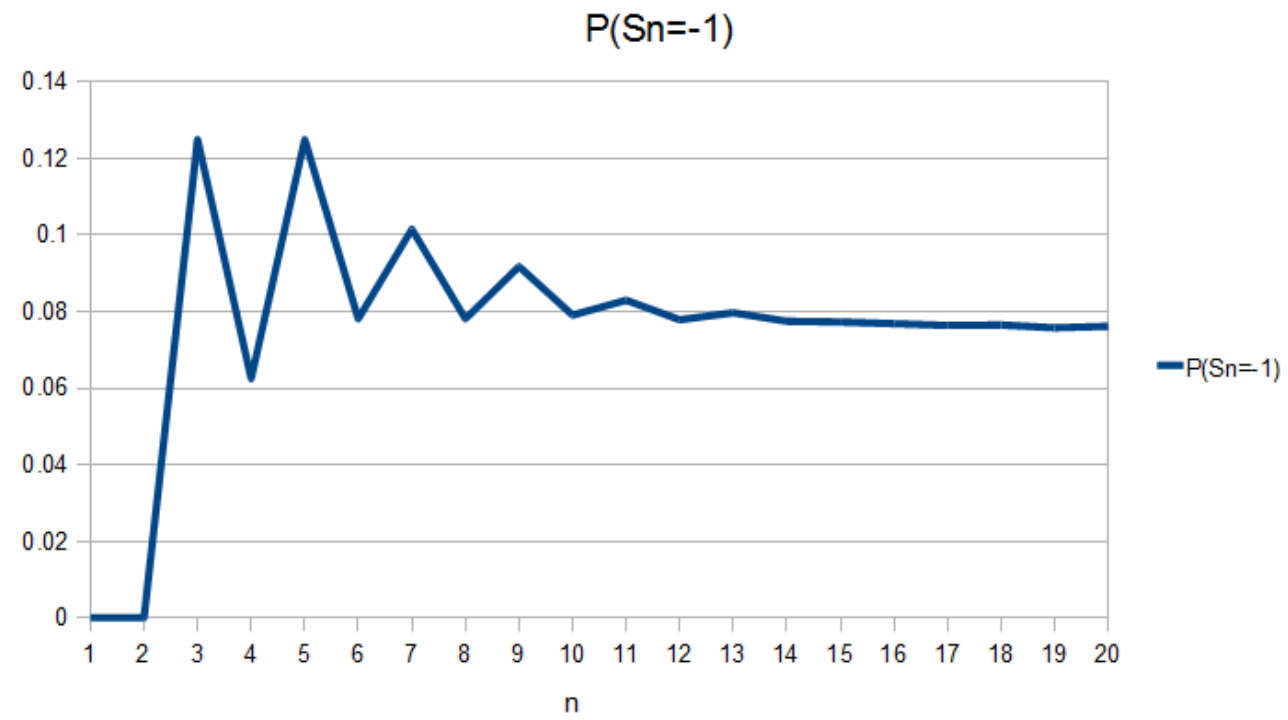

Figura 2.4: Convergência 3 de $\mu\left(S_{n}=-1\right)$.

Os exemplos anteriores mostraram que $\mu\left(S_{n}=k\right)$ pode convergir mesmo com $k$ negativo num processo sem gramática completa.

\subsection{Limite da Esperança de $S_{n}$}

Seguindo a linha de trabalho proposta, vamos analisar o limite da esperança de $S_{n}$. É claro que o fato de conhecer a forma explícita de tal distribuição talvez nos daria um resultado mais direto. Mas a dificuldade (e a beleza) desse problema se encontra justamente no fato de $S_{n}$ não possuir uma forma explícita simples de distribuição de probabilidade. $\mathrm{Ou}$ seja, não se conhece uma expressão $F_{S_{n}}$ para a distribuição de $S_{n}$.

Primeiro, vamos apresentar o enunciado do teorema, e logo, os lemas que sustentarão a prova.

Teorema 2.10. Seja $\mu$ uma medida de probabilidade definida em $\chi^{\mathbb{N}}$ para um processo $\beta$ mixing com gramática completa. Então,

$$
\left|\mathbb{E}\left(S_{n}\right)-\sum_{k=1}^{\infty}\left(\mathbb{E}\left(\mu\left(X_{1}^{k}\right)\right)+a_{k}\right)\right| \leq K_{\rho}^{\prime} \sum_{i=\lfloor n / 8\rfloor}^{\infty} i \varepsilon_{\beta}(i),
$$

onde $K_{\rho}^{\prime}$ é uma contante que depende só de $\rho$ e $\varepsilon_{\beta}(i)$ e $a_{k}$ como foram definidos no Teorema 2.1. Se, além do anterior, o processo é tal que $i \ln i \beta(i)$ é somável, então

$$
\lim _{n \rightarrow \infty} \sum_{i=\lceil n / 8\rceil}^{\infty} i \varepsilon_{\beta}(i)=0
$$

o que implica que

$$
\lim _{n \rightarrow \infty} \mathbb{E}\left(S_{n}\right)=\sum_{k=1}^{\infty}\left(\mathbb{E}\left(\mu\left(X_{1}^{k}\right)\right)+a_{k}\right) .
$$

Para a prova do teorema vamos usar o seguinte lema. 
Lema 2.11. Sejam $\mu$ uma medida de probabilidade definida em $\chi^{\mathbb{N}}$ para um processo $\beta$ mixing e $0<b<1$ uma constante. Então,

$$
\sum_{k=\lfloor b n\rfloor}^{n-1} \mu\left(S_{n} \geq k\right) \leq n K_{b, \rho} \sum_{i=\left\lfloor b^{\prime} n\right\rfloor}^{\lceil n(1-b)\rceil} \inf _{1 \leq m<i}\left(\rho^{m}+\tilde{\beta}_{m}(i)\right)
$$

onde $K_{\rho, b}$ é uma constante que depende de $\rho$ e de $b$ e $b^{\prime}=\min \{b / 2,(1-b) / 2\}$.

Demonstração. Usando a relação de dualidade 1.18 e o fato do processo ter gramática completa, temos que

$$
\begin{aligned}
\mu\left(S_{n} \geq k\right) & =\mu\left(\bigcup_{i=k}^{n-1} R_{n}(i)\right) \\
& =\mu\left(\bigcup_{i=1}^{n-k} B_{n}(i)\right) .
\end{aligned}
$$

Somando em $i$ desde $\lfloor b n\rfloor$ até $n-1$, obtemos que,

$$
\begin{aligned}
\sum_{k=\lfloor b n\rfloor}^{n-1} \mu\left(S_{n} \geq k\right) & =\sum_{k=\lfloor b n\rfloor}^{n-1} \mu\left(\bigcup_{i=1}^{n-k} B_{n}(i)\right) \\
& =\sum_{k=1}^{\lceil n(1-b)\rceil} \mu\left(\bigcup_{i=1}^{k} B_{n}(i)\right) .
\end{aligned}
$$

Vamos limitar superiormente a desigualdade (2.55) usando a união maior, ou seja, $\mu\left(\bigcup_{i=1}^{\lceil n(1-b)\rceil} B_{n}(i)\right)$.

$$
\begin{aligned}
\sum_{k=\lfloor b n\rfloor}^{n-1} \mu\left(S_{n} \geq k\right) & \leq\lceil n(1-b)\rceil \mu\left(\bigcup_{i=1}^{\lceil n(1-b)\rceil} B_{n}(i)\right) \\
& \leq\lceil n(1-b)\rceil C_{b, \rho} \sum_{i=\left\lfloor b^{\prime} n\right\rfloor}^{\lceil n(1-b)\rceil} \inf _{1 \leq m<i}\left(\rho^{m}+\tilde{\beta}_{m}(i)\right)
\end{aligned}
$$

onde a última linha foi obtida aplicando o Lema 2.8. Aqui $b^{\prime}=\min \{b / 2,(1-b) / 2\}$, com o qual acaba a prova do lema, pois reescrevendo as constantes na expressão à direita na desigualdade (2.56) chegamos ao nosso resultado.

\section{Demostração do Teorema 2.10}

Dado que o nosso processo tem gramática completa, os valores assumidos pela variável aleatória $S_{n}$ estão entre 0 e $n-1$, portanto, a esperança de $S_{n}$ pode ser escrita da seguinte maneira,

$$
\mathbb{E}\left(S_{n}\right)=\sum_{k=1}^{n-1} \mu\left(S_{n} \geq k\right)
$$


Dessa forma, podemos substituir esta expressão na seguinte equação, obtendo que

$$
\begin{aligned}
\left|\mathbb{E}\left(S_{n}\right)-\sum_{k=1}^{\infty}\left(\mathbb{E}\left(\mu\left(X_{1}^{k}\right)\right)+a_{k}\right)\right| & =\left|\sum_{k=1}^{n-1} \mu\left(S_{n} \geq k\right)-\sum_{k=1}^{\infty}\left(\mathbb{E}\left(\mu\left(X_{1}^{k}\right)\right)+a_{k}\right)\right| \\
& \leq \sum_{k=1}^{n-1}\left|\mu\left(S_{n} \geq k\right)-\mathbb{E}\left(\mu\left(X_{1}^{k}\right)\right)-a_{k}\right|+\sum_{k=n}^{\infty}\left(\mathbb{E}\left(\mu\left(X_{1}^{k}\right)\right)+a_{k}\right) .
\end{aligned}
$$

Agora analisaremos cada somatório da desigualdade (2.57) separadamente. Começamos dividendo o primeiro termo para usar a desigualdade triangular obtendo que

$$
\sum_{k=1}^{n-1}\left|\mu\left(S_{n} \geq k\right)-\mathbb{E}\left(\mu\left(X_{1}^{k}\right)\right)-a_{k}\right| \leq \sum_{k=1}^{\lfloor n / 4\rfloor}\left|\mu\left(S_{n} \geq k\right)-\mathbb{E}\left(\mu\left(X_{1}^{k}\right)\right)-a_{k}\right|+\sum_{k=\lfloor n / 4\rfloor+1}^{n-1}\left|\mu\left(S_{n} \geq k\right)-\mathbb{E}\left(\mu\left(X_{1}^{k}\right)\right)-a_{k}\right|
$$

Nós concentraremos no primeiro somatório à direita na inequação (2.58). Pelo Teorema 2.1,

$$
\begin{aligned}
\sum_{k=1}^{\lfloor n / 4\rfloor}\left|\mu\left(S_{n} \geq k\right)-\mathbb{E}\left(\mu\left(X_{1}^{k}\right)\right)-a_{k}\right| & \leq K_{\rho} \sum_{k=1}^{\lfloor n / 4\rfloor} \sum_{i=\lfloor n / 8\rfloor}^{\infty} \inf _{1 \leq m<i}\left(\rho^{m}+\tilde{\beta}_{m}(i)\right) \\
& =K_{\rho}\left\lfloor\frac{n}{4}\right\rfloor \sum_{i=\lfloor n / 8\rfloor}^{\infty} \inf _{1 \leq m<i}\left(\rho^{m}+\tilde{\beta}_{m}(i)\right)
\end{aligned}
$$

Passamos a analisar o segundo somatório da desigualdade (2.58). Pela desigualdade triangular, temos

$$
\sum_{k=\lfloor n / 4\rfloor+1}^{n-1}\left|\mu\left(S_{n} \geq k\right)-\mathbb{E}\left(\mu\left(X_{1}^{k}\right)\right)-a_{k}\right| \leq \sum_{k=\lfloor n / 4\rfloor+1}^{n-1} \mu\left(S_{n} \geq k\right)+\sum_{k=\lfloor n / 4\rfloor+1}^{n-1}\left(\mathbb{E}\left(\mu\left(X_{1}^{k}\right)\right)+a_{k}\right)
$$

Vamos estudar separadamente cada somatório na inequação (2.60). Pelo Lema 2.11, tomando $b=1 / 4$, afirmamos que

$$
\sum_{k=\lfloor n / 4\rfloor+1}^{n-1} \mu\left(S_{n} \geq k\right) \leq n K_{\rho} \sum_{i=\lfloor n / 8\rfloor}^{\lceil 3 n / 4\rceil} \inf _{1 \leq m<i}\left(\rho^{m}+\tilde{\beta}_{m}(i)\right)
$$

Por outro lado, somamos o segundo somatório na desigualdade (2.57) com o segundo somatório da desigualdade (2.60) e afirmamos que

$$
\sum_{k=\lfloor n / 4\rfloor+1}^{\infty}\left(\mathbb{E}\left(\mu\left(X_{1}^{k}\right)\right)+a_{k}\right) \leq \sum_{k=\lfloor n / 4\rfloor+1}^{\infty} \sum_{i=k}^{\infty} \mathbb{E}\left(\mu\left(X_{1}^{i}\right)\right)
$$


pois $a_{k} \leq \sum_{i=k+1}^{\infty} \mathbb{E}\left(\mu\left(X_{1}^{i}\right)\right)$. Vamos desenvolver o último somatório.

$$
\begin{aligned}
\sum_{k=\lfloor n / 4\rfloor+1}^{\infty} \sum_{i=k}^{\infty} \mathbb{E}\left(\mu\left(X_{1}^{i}\right)\right) & =\sum_{i=\lfloor n / 4\rfloor+1}^{\infty} \mathbb{E}\left(\mu\left(X_{1}^{i}\right)\right)+\sum_{i=\lfloor n / 4\rfloor+2}^{\infty} \mathbb{E}\left(\mu\left(X_{1}^{i}\right)\right)+\cdots+\sum_{i=n}^{\infty} \mathbb{E}\left(\mu\left(X_{1}^{i}\right)\right)+\ldots \\
& =\mathbb{E}\left(\mu\left(X_{1}^{\lfloor n / 4\rfloor+1}\right)\right)+2 \mathbb{E}\left(\mu\left(X_{1}^{\lfloor n / 4\rfloor+2}\right)\right)+\cdots+\left\lceil\frac{3 n}{4}\right\rceil \mathbb{E}\left(\mu\left(X_{1}^{n}\right)\right)+\ldots \\
& =\sum_{i=\lfloor n / 4\rfloor+1}^{\infty}\left(i-\left\lfloor\frac{n}{4}\right\rfloor\right) \mathbb{E}\left(\mu\left(X_{1}^{i}\right)\right) \\
& <\sum_{i=\lfloor n / 4\rfloor+1}^{\infty} i \mathbb{E}\left(\mu\left(X_{1}^{i}\right)\right)
\end{aligned}
$$

Acharemos agora um limitante superior para $i \mathbb{E}\left(\mu\left(X_{1}^{i}\right)\right)$. Usando a expressão (2.16) temos que,

$$
\sum_{i=\lfloor n / 4\rfloor+1}^{\infty} i \mathbb{E}\left(\mu\left(X_{1}^{i}\right)\right) \leq \sum_{i=\lfloor n / 4\rfloor+1}^{\infty} i \inf _{1 \leq m<i}\left(\rho^{m}+\frac{\beta_{m}(i)}{2(1-\rho)}\right) .
$$

De forma a concluir a primeira parte do teorema, somamos as desigualdades (2.59), (2.61) e (2.63) e obtemos o resultado procurado.

Agora, vamos a provar a convergência. Para isso, tomamos $m=\log _{\rho}(1 / f(i))$, onde $f(i)=i^{2}(\ln i)^{1+\epsilon}$ para algum $\epsilon>0$. Nós queremos encontrar

$$
\lim _{n \rightarrow \infty} \sum_{i=\left\lfloor\frac{n}{8}\right\rfloor}^{\infty} \inf _{1 \leq m<i} i\left(\rho^{m}+\tilde{\beta}_{m}(i)\right) \leq \lim _{n \rightarrow \infty} \sum_{i=\left\lfloor\frac{n}{8}\right\rfloor}^{\infty} \frac{i}{f(i)}+\lim _{n \rightarrow \infty} \sum_{i=\left\lfloor\frac{n}{8}\right\rfloor}^{\infty} i \tilde{\beta}\left(\left\lfloor\frac{i}{\log _{\rho}(1 / f(i))}\right\rfloor-1\right) .
$$

O primeiro limite à direita na desigualdade é igual a zero porque $1 / i(\ln i)^{1+\epsilon}$ é somável. Assim, voltaremos nossa atenção à soma com termos $\beta$. O limite que nós procuramos, é igual ao limite da expressão $\sum_{i=\left\lfloor\frac{n}{8}\right\rfloor}^{\infty} \tilde{\beta}\left(\left\lfloor i \rho_{1} / \ln i\right\rfloor\right)$, onde $\rho_{1}=-\ln \rho / 3$. Note que $\rho_{1}>0$ porque $\ln \rho<0$. Vamos provar que este limite é zero. Comecemos afirmando que

$$
\ln f(i)<3 \ln i
$$

pois $\ln f(i)=2 \ln i+(1+\epsilon) \ln (\ln i), \ln i<i$ para todo $i$ e $\epsilon<1$. Portanto,

$$
\frac{i}{\ln f(i)}>\frac{i}{3 \ln i} \text {. }
$$

Usando o mesmo procedimento feito para contar os inteiros $h$ que satisfaziam a condição (2.46) na prova do Teorema 2.1 se obtém uma desigualdade similar à apresentada na expressão (2.48), com ajuda das equações (2.65) e (2.45), concluindo que o número de inteiros $h$ que satisfazem (2.46), neste caso é

$$
h<\frac{3}{\rho_{1}} \ln (i+1)
$$


Como $\beta$ é uma função decrescente,

$$
\sum_{i=\lfloor n / 8\rfloor}^{\infty} i \tilde{\beta}\left(\left\lfloor\frac{i \rho_{1}}{\ln i}\right\rfloor\right) \leq \sum_{i=\left\lfloor\frac{\lfloor n / 8\rfloor \rho_{1}}{\ln \lfloor n / 8\rfloor}\right\rfloor}^{\infty} \frac{3 i \ln (i+1)}{\rho_{1}} \tilde{\beta}(i)=\frac{3}{\rho_{1}} \sum_{i=\left\lfloor\frac{\left.\lfloor n / 8\rfloor \rho_{1}\right\rfloor}{\ln \lfloor n / 8\rfloor}\right\rfloor}^{\infty} i \ln (i+1) \tilde{\beta}(i) .
$$

E como por hipótese $i \ln i \beta(i)$ é somável, obtemos o limite zero que procurávamos, com o qual finalizamos nossa prova.

\subsection{Limite do Segundo Momento Finito de $S_{n}$}

Seguindo a estrutura das seções anteriores, começamos enunciando o teorema principal, o seu corolário e a seguir o lema que sustenta as provas.

Teorema 2.12. Seja $\mu$ uma medida de probabilidade definida em $\chi^{\mathbb{N}}$ para um processo $\beta$ mixing com gramática completa. Então,

$$
\left|\mathbb{E}\left(S_{n}^{2}\right)-\sum_{k=1}^{\infty}(2 k-1)\left(\mathbb{E}\left(\mu\left(X_{1}^{k}\right)\right)+a_{k}\right)\right| \leq K_{\rho}^{\prime \prime} \sum_{i=\lfloor n / 8\rfloor}^{\infty} i^{2} \varepsilon_{\beta}(i),
$$

onde $K_{\rho}^{\prime \prime}$ é uma constante que depende só de $\rho$ e $\varepsilon_{\beta}(i)$ e $a_{k}$ como foram definidos no Teorema 2.1. Se, além do anterior, o processo é tal que $i^{2} \ln i \beta(i)$ é somável, então

$$
\lim _{n \rightarrow \infty} \sum_{i=\lceil n / 8\rceil}^{\infty} i^{2} \varepsilon_{\beta}(i)=0
$$

o que implica que

$$
\lim _{n \rightarrow \infty} \mathbb{E}\left(S_{n}^{2}\right)=\sum_{k=1}^{\infty}\left(\mathbb{E}\left(\mu\left(X_{1}^{k}\right)\right)+a_{k}\right)
$$

O interesse que tem a quantidade $\mathbb{E}\left(S_{n}^{2}\right)$ vem da possibilidade que este oferece no cálculo da variância de $S_{n}$. Motivados por isso, damos o seguinte corolário.

Corolário 2.13. Sob as hipóteses do Teorema 2.12, temos que

$$
\lim _{n \rightarrow \infty} \operatorname{Var}\left(S_{n}\right)=\sum_{k=1}^{\infty}(2 k-1)\left[\mathbb{E}\left(\mu\left(X_{1}^{k}\right)\right)+a_{k}\right]-\left(\sum_{k=1}^{\infty}\left(\mathbb{E}\left(\mu\left(X_{1}^{k}\right)\right)+a_{k}\right)\right)^{2} .
$$

As provas do teorema principal desta seção e do corolário serão baseadas no seguinte lema.

Lema 2.14. Sejam $\mu$ uma medida de probabilidade definida em $\chi^{\mathbb{N}}$ para um processo $\beta$ mixing e $0<d<1$ uma constante. Então,

$$
\sum_{k=\lfloor d n\rfloor}^{n-1} k \mu\left(S_{n} \geq k\right) \leq n^{2} K_{\rho, d} \sum_{i=\left\lfloor d^{\prime} n\right\rfloor}^{\lceil n(1-d)\rceil} \inf _{1 \leq m<i}\left(\rho^{m}+\tilde{\beta}_{m}(i)\right)
$$

onde $K_{\rho, d}$ é uma constante que depende de $\rho$ e de $d$, e $d^{\prime}=\min \{d / 2,(1-d) / 2\}$. 
Demonstração. Como nosso processo tem gramática completa, usando a equação (2.54) temos que,

$$
\begin{aligned}
\sum_{k=\lfloor d n\rfloor}^{n-1} k \mu\left(S_{n} \geq k\right) & =\sum_{k=\lfloor d n\rfloor}^{n-1} k \mu\left(\bigcup_{i=1}^{n-k} B_{n}(i)\right) \\
& =\sum_{k=1}^{\lceil n(1-d)\rceil}(n-k) \mu\left(\bigcup_{i=1}^{k} B_{n}(i)\right) .
\end{aligned}
$$

Vamos limitar a equação (2.69) usando a união maior, ou seja, $\mu\left(\bigcup_{i=1}^{\lceil n(1-d)\rceil} B_{n}(i)\right)$.

$$
\begin{aligned}
\sum_{k=\lfloor d n\rfloor}^{n-1} k \mu\left(S_{n} \geq k\right) & <n \sum_{k=1}^{\lceil n(1-d)\rceil} \mu\left(\bigcup_{i=1}^{k} B_{n}(i)\right) \\
& \leq n\lceil n(1-d)\rceil \mu\left(\bigcup_{i=1}^{\lceil n(1-d)\rceil} B_{n}(i)\right) \\
& \leq n^{2} C_{\rho, d} \sum_{i=\left\lfloor d^{\prime} n\right\rfloor}^{\lceil n(1-d)\rceil} \inf _{1 \leq m<i}\left(\rho^{m}+\tilde{\beta}_{m}(i)\right),
\end{aligned}
$$

onde a última linha foi obtida aplicando o Lema 2.8 e $d^{\prime}=\min \{d / 2,(1-d) / 2\}$, ficando provado nosso lema.

\section{Demostração do Teorema 2.12}

Dado que o nosso processo tem gramática completa, o segundo momento finito de $S_{n}$ pode ser escrita como o somatório de $k$ desde 1 até $n-1$ da seguinte maneira,

$$
\begin{aligned}
\mathbb{E}\left(S_{n}\right) & =\sum_{k=1}^{n-1} k^{2} \mu\left(S_{n}=k\right) \\
& =\sum_{k=1}^{n-1}(2 k-1) \mu\left(S_{n} \geq k\right) .
\end{aligned}
$$

Dessa forma, podemos substituir a equação (2.70) na seguinte expressão:

$$
\begin{aligned}
\left|\mathbb{E}\left(S_{n}^{2}\right)-\sum_{k=1}^{\infty}(2 k-1)\left(\mathbb{E}\left(\mu\left(X_{1}^{k}\right)\right)+a_{k}\right)\right| & =\left|\sum_{k=1}^{n-1}(2 k-1) \mu\left(S_{n} \geq k\right)-\sum_{k=1}^{\infty}(2 k-1)\left(\mathbb{E}\left(\mu\left(X_{1}^{k}\right)\right)+a_{k}\right)\right| \\
\leq & \sum_{k=1}^{n-1}(2 k-1)\left|\mu\left(S_{n} \geq k\right)-\mathbb{E}\left(\mu\left(X_{1}^{k}\right)\right)-a_{k}\right| \\
& +\sum_{k=n}^{\infty}(2 k-1)\left(\mathbb{E}\left(\mu\left(X_{1}^{k}\right)\right)+a_{k}\right) .
\end{aligned}
$$

Agora analisaremos cada somatório da desigualdade (2.71) separadamente. Começamos di- 
videndo o primeiro somatório com o objetivo de aplicar o Teorema 2.1. Portanto,

$$
\begin{array}{r}
\sum_{k=1}^{n-1}(2 k-1)\left|\mu\left(S_{n} \geq k\right)-\mathbb{E}\left(\mu\left(X_{1}^{k}\right)\right)-a_{k}\right| \leq \sum_{k=1}^{\lfloor n / 4\rfloor}(2 k-1)\left|\mu\left(S_{n} \geq k\right)-\mathbb{E}\left(\mu\left(X_{1}^{k}\right)\right)-a_{k}\right| \\
+\sum_{k=\lfloor n / 4\rfloor+1}^{n-1}(2 k-1)\left|\mu\left(S_{n} \geq k\right)-\mathbb{E}\left(\mu\left(X_{1}^{k}\right)\right)-a_{k}\right| .
\end{array}
$$

Nós concentraremos no primeiro somatório à direita na inequação (2.72). Aplicando o Teorema 2.1, obtemos que

$$
\begin{aligned}
\sum_{k=1}^{\lfloor n / 4\rfloor}(2 k-1)\left|\mu\left(S_{n} \geq k\right)-\mathbb{E}\left(\mu\left(X_{1}^{k}\right)\right)-a_{k}\right| & \leq K_{\rho} \sum_{k=1}^{\lfloor n / 4\rfloor}(2 k-1) \sum_{i=\lfloor n / 8\rfloor}^{\infty} \inf _{1 \leq m<i}\left(\rho^{m}+\tilde{\beta}_{m}(i)\right) \\
& \leq K_{\rho}\left\lfloor\frac{n}{4}\right\rfloor^{2} \sum_{i=\lfloor n / 8\rfloor}^{\infty} \inf _{1 \leq m<i}\left(\rho^{m}+\tilde{\beta}_{m}(i)\right)
\end{aligned}
$$

Por outro lado, pela desigualdade triangular, temos que

$$
\begin{aligned}
\sum_{k=\lfloor n / 4\rfloor+1}^{n-1}(2 k-1)\left|\mu\left(S_{n} \geq k\right)-\mathbb{E}\left(\mu\left(X_{1}^{k}\right)\right)-a_{k}\right| & \leq \sum_{k=\lfloor n / 4\rfloor+1}^{n-1}(2 k-1) \mu\left(S_{n} \geq k\right) \\
& +\sum_{k=\lfloor n / 4\rfloor+1}^{n-1}(2 k-1)\left(\mathbb{E}\left(\mu\left(X_{1}^{k}\right)\right)+a_{k}\right) .
\end{aligned}
$$

Vamos estudar separadamente cada somatório na inequação (2.74). Pelo Lema 2.14, tomando $d=1 / 4$, afirmamos que

$$
\sum_{k=\lfloor n / 4\rfloor+1}^{n-1}(2 k-1) \mu\left(S_{n} \geq k\right) \leq n^{2} \tilde{K}_{\rho} \sum_{i=\lfloor n / 8\rfloor}^{\lceil 3 n / 4\rceil} \inf _{1 \leq m<i}\left(\rho^{m}+\tilde{\beta}_{m}(i)\right) .
$$

Por outro lado, somamos o segundo somatório na desigualdade (2.71) com o segundo somatório da desigualdade (2.74) e afirmamos que

$$
\sum_{k=\lfloor n / 4\rfloor+1}^{\infty}(2 k-1)\left(\mathbb{E}\left(\mu\left(X_{1}^{k}\right)\right)+a_{k}\right) \leq \sum_{k=\lfloor n / 4\rfloor+1}^{\infty}(2 k-1) \sum_{i=k}^{\infty} \mathbb{E}\left(\mu\left(X_{1}^{i}\right)\right)
$$

pois $a_{k} \leq \sum_{i=k+1}^{\infty} \mathbb{E}\left(\mu\left(X_{1}^{i}\right)\right)$. Vamos desenvolver o último somatório.

$$
\begin{gathered}
\sum_{k=\lfloor n / 4\rfloor+1}^{\infty}(2 k-1) \sum_{i=k}^{\infty} \mathbb{E}\left(\mu\left(X_{1}^{i}\right)\right)= \\
\left(2\left\lfloor\frac{n}{4}\right\rfloor+1\right) \sum_{i=\lfloor n / 4\rfloor+1}^{\infty} \mathbb{E}\left(\mu\left(X_{1}^{i}\right)\right)+\left(2\left\lfloor\frac{n}{4}\right\rfloor+3\right) \sum_{i=\lfloor n / 4\rfloor+2}^{\infty} \mathbb{E}\left(\mu\left(X_{1}^{i}\right)\right)+\cdots+(2 n-1) \sum_{i=n}^{\infty} \mathbb{E}\left(\mu\left(X_{1}^{i}\right)\right)+\ldots
\end{gathered}
$$


Portanto,

$$
\sum_{k=\lfloor n / 4\rfloor+1}^{n-1}(2 k-1) \sum_{i=k}^{\infty} \mathbb{E}\left(\mu\left(X_{1}^{i}\right)\right) \leq \sum_{i=\lfloor n / 4\rfloor+1}^{\infty} i^{2} \mathbb{E}\left(\mu\left(X_{1}^{i}\right)\right) .
$$

Acharemos agora um limitante superior para $i^{2} \mathbb{E}\left(\mu\left(X_{1}^{i}\right)\right)$. Usando a equação (2.16), concluímos que

$$
\sum_{i=\lfloor n / 4\rfloor+1}^{\infty} i^{2} \mathbb{E}\left(\mu\left(X_{1}^{i}\right)\right) \leq C_{\rho} \sum_{i=\lfloor n / 4\rfloor+1}^{\infty} i^{2} \inf _{1 \leq m<i}\left(\rho^{m}+\beta_{m}(i)\right)
$$

Para concluir a primeira parte do teorema, somamos as desigualdades (2.73), (2.75) e (2.76) e obtemos o resultado procurado.

Para provar a convergência, vamos tomar $m=\log _{\rho}(1 / f(i))$, onde $f(i)=i^{3}(\ln i)^{1+\epsilon}$ para algum $\epsilon$ entre 0 e 1 . Nós queremos encontrar

$$
\lim _{n \rightarrow \infty} \sum_{i=\left\lfloor\frac{n}{8}\right\rfloor}^{\infty} \inf _{1 \leq m<i} i^{2}\left(\rho^{m}+\tilde{\beta}_{m}(i)\right) \leq \lim _{n \rightarrow \infty} \sum_{i=\left\lfloor\frac{n}{8}\right\rfloor}^{\infty} \frac{i^{2}}{f(i)}+\lim _{n \rightarrow \infty} \sum_{i=\left\lfloor\frac{n}{8}\right\rfloor}^{\infty} i^{2} \tilde{\beta}\left(\left\lfloor\frac{i}{\log _{\rho}(1 / f(i))}\right\rfloor-1\right) .
$$

O primeiro limite à direita na desigualdade anterior é igual a zero porque $1 / i(\ln i)^{1+\epsilon}$ é somável. Assim, voltaremos nossa atenção à soma com termos $\beta$. O limite que nós procuramos, é igual ao limite da expressão $\sum_{i=\left\lfloor\frac{n}{8}\right\rfloor}^{\infty} \tilde{\beta}\left(\left\lfloor i \rho_{2} / \ln i\right\rfloor\right)$, onde $\rho_{2}=-\ln \rho / 4$. Note que $\rho_{2}>0$ porque $\ln \rho<0$. Vamos provar que este limite é zero. Comecemos afirmando que

$$
\ln f(i)<4 \ln i
$$

pois $\ln f(i)=3 \ln i+(1+\epsilon) \ln (\ln i), \ln i<i$ para todo $i$ e $\epsilon<1$. Portanto,

$$
\frac{i}{\ln f(i)}>\frac{i}{4 \ln i}
$$

Usando o mesmo procedimento feito para contar os inteiros $h$ que satisfaziam a condição (2.46) na prova do Teorema 2.1 se obtém uma desigualdade similar à apresentada na expressão (2.48), com ajuda das equações (2.78) e (2.45), concluindo que o número de inteiros $h$ que satisfazem (2.46), neste caso é

$$
h<\frac{4}{\rho_{1}} \ln (i+1)
$$

Como $\beta$ é uma função decrescente,

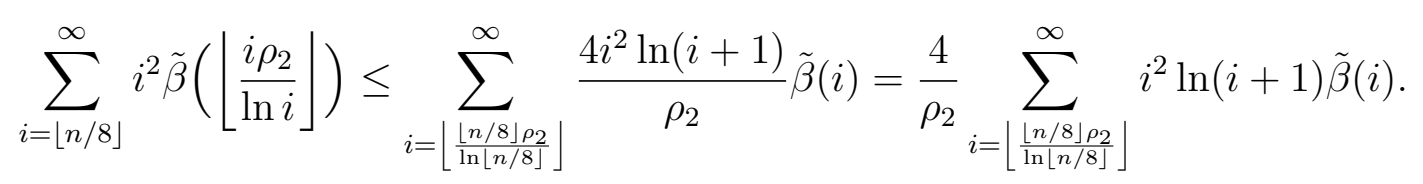

E como por hipótese $i^{2} \ln i \beta(i)$ é somável, obtemos o limite zero que procurávamos, com o qual finalizamos nossa prova.

Demostração do Corolário 2.13. A prova desde corolário é imediata. Pois basta substituir os limites dados nos Teoremas 2.10 e 2.12 na muito conhecida equação $\operatorname{Var}\left(S_{n}\right)=\mathbb{E}\left(S_{n}^{2}\right)$ - 
$\left(\mathbb{E}\left(S_{n}\right)\right)^{2}$

\subsection{Resultados condicionais}

\subsubsection{Probabilidade condicional}

As variáveis aleatórias $\left\{S_{n}\right\}_{n \in \mathbb{N}}$ são definidas comparando uma palavra no começo de sequências infinitas e o lugar onde esta palavra aparece de novo. Se soubéssemos o começo da palavra iniciante, a distribuições de probabilidade de $S_{n}$ deveriam ser, intuitivamente, independentes. E isto intui-se pelo fato do processo $\beta$-mixing perder memória com o passar do tempo. Nós, então, nos perguntamos pelo limite da distribuição de $S_{n}$ dado que se conhece o processo até um tempo $t_{n}$. Nos seguintes teoremas consideramos palavras $a_{1}^{t_{n}}$ com medida positiva.

Teorema 2.15. Seja $\mu$ uma medida de probabilidade definida sobre $\chi^{\mathbb{N}}$ para um processo $\beta$-mixing com gramática completa. Consideremos $k, n$, e $t_{n} \in \mathbb{N}$ tal que $2 k \leq t_{n}<n / 2$ para todo n. Então,

$$
\left|\mu\left(S_{n} \geq k \mid X_{1}^{t_{n}}=a_{1}^{t_{n}}\right)-\mu\left(a_{1}^{k}\right)-\alpha_{k}\right| \leq \frac{K_{1}(\rho)}{\mu\left(a_{1}^{t_{n}}\right)} \sum_{i=c_{n}^{\prime}}^{\infty} \inf _{1 \leq m<i}\left(\rho^{m}+\tilde{\beta}_{m}(i)\right),
$$

onde $K_{1}(\rho)$ é uma constante que depende somente de $\rho, c_{n}^{\prime}=\min \left\{\left\lfloor t_{n} / 2\right\rfloor,\left\lfloor\left(n-t_{n}\right) / 2\right\rfloor\right\}$ e

$$
\alpha_{k}=\sum_{i=k+1}^{\infty} \mu\left(a_{1}^{i}: a_{1}^{i} \notin \bigcup_{j=k}^{i-1} R_{j}(j)\right)
$$

Se, além do anterior, o processo $\beta$-mixing é tal que $\ln i \beta(i)$ é somável e $t_{n} / n$ tende a zero quando $n$ diverge, vale que

$$
\lim _{n \rightarrow \infty} \mu\left(S_{n} \geq k \mid X_{1}^{t_{n}}=a_{1}^{t_{n}}\right)=\mu\left(a_{1}^{k}\right)+\alpha_{k} .
$$

Demonstração. Pela definição da probabilidade condicional e pela gramática completa do processo, temos que

$$
\mu\left(S_{n} \geq k \mid X_{1}^{t_{n}}=a_{1}^{t_{n}}\right)=\frac{\mu\left(S_{n} \geq k \cap X_{1}^{t_{n}}=a_{1}^{t_{n}}\right)}{\mu\left(a_{1}^{t_{n}}\right)}=\frac{\mu\left(\bigcup_{i=k}^{n-1} R_{i}(i) \cap X_{1}^{t_{n}}=a_{1}^{t_{n}}\right)}{\mu\left(a_{1}^{t_{n}}\right)} .
$$

As equações (2.34) e (2.35) são válidas mesmo trocando $\lfloor n / 8\rfloor$ por $t_{n}$, logo, afirmamos que,

$$
\begin{aligned}
& \mu\left(S_{n} \geq k \mid X_{1}^{t_{n}}=a_{1}^{t_{n}}\right)= \\
& \frac{1}{\mu\left(a_{1}^{t_{n}}\right)}\left[\mu\left(R_{n}(k) \cap X_{1}^{t_{n}}=a_{1}^{t_{n}}\right)+\sum_{i=k+1}^{t_{n}} \mu\left(R_{n}(i) \cap X_{1}^{t_{n}}=a_{1}^{t_{n}} \backslash \bigcup_{j=k}^{i-1} R_{n}(j)\right)+\mu\left(\bigcup_{i=t_{n}+1}^{n-1} R_{n}(i) \cap X_{1}^{t_{n}}=a_{1}^{t_{n}}\right)\right] .
\end{aligned}
$$

Vamos denotar por

$$
\alpha_{k, n}=\sum_{i=k+1}^{t_{n}} \mu\left(a_{1}^{i}: a_{1}^{i} \notin \bigcup_{j=k}^{i-1} R_{j}(j)\right)
$$


somando e substituindo $\alpha_{k, n}$, é verdade que

$$
\left|\mu\left(S_{n} \geq k \mid X_{1}^{t_{n}}=a_{1}^{t_{n}}\right)-\mu\left(a_{1}^{k}\right)-\alpha_{k}\right|=\left|\mu\left(S_{n} \geq k \mid X_{1}^{t_{n}}=a_{1}^{t_{n}}\right)-\mu\left(a_{1}^{k}\right)-\alpha_{k}+\alpha_{k, n}-\alpha_{k, n}\right| .
$$

Desse modo, pela equação (2.82) e a desigualdade triangular,

$$
\begin{aligned}
\left|\mu\left(S_{n} \geq k \mid X_{1}^{t_{n}}=a_{1}^{t_{n}}\right)-\mu\left(a_{1}^{k}\right)-\alpha_{k}\right| & \leq\left|\frac{1}{\mu\left(a_{1}^{t_{n}}\right)} \mu\left(R_{n}(k) \cap X_{1}^{t_{n}}=a_{1}^{t_{n}}\right)-\mu\left(a_{1}^{k}\right)\right| \\
& +\left|\frac{1}{\mu\left(a_{1}^{t_{n}}\right)} \sum_{i=k+1}^{t_{n}} \mu\left(R_{n}(i) \cap X_{1}^{t_{n}}=a_{1}^{t_{n}} \backslash \bigcup_{j=k}^{i-1} R_{n}(j)\right)-\alpha_{k, n}\right| \\
& +\left|\alpha_{k}-\alpha_{k, n}\right| \\
& +\frac{1}{\mu\left(a_{1}^{t_{n}}\right)} \mu\left(\bigcup_{i=t_{n}+1}^{n-1} R_{n}(i) \cap X_{1}^{t_{n}}=a_{1}^{t_{n}}\right) .
\end{aligned}
$$

Vamos limitar superiormente cada termo à direita na desigualdade anterior. Começamos com a equação (2.83). Se $\omega \in R_{n}(k) \cap X_{1}^{t_{n}}=a_{1}^{t_{n}}$, então $\omega=a_{1}^{t_{n}} x_{t_{n}+1}^{n-t_{n}-k} a_{1}^{k}$, onde $a_{1}^{t_{n}}=a_{1}^{k} a_{k+1}^{t_{n}}$. Desta maneira, se nós conhecemos $a_{1}^{t_{n}}$, também conhecemos $a_{1}^{k}$. Removamos o bloco $x_{t_{n}+1}^{n-t_{n}-k}$ de $\omega$. Assim, temos que

$$
\mu(\omega) \leq \mu\left(a_{1}^{t_{n}}, a_{1}^{k}\right) .
$$

Mais ainda, como $a_{1}^{t_{n}}$ é conhecido, afirmamos que

$$
\mu\left(R_{n}(k) \cap X_{1}^{t_{n}}=a_{1}^{t_{n}}\right)=\sum_{\omega \in R_{n}(k) \cap X_{1}^{t_{n}}=a_{1}^{t_{n}}} \mu(\omega) \leq \mu\left(a_{1}^{t_{n}}, a_{1}^{k}\right) .
$$

A propriedade $\beta$-mixing do processo nos diz que,

$$
\sum_{a_{1}^{t_{n}}, a_{1}^{k}}\left|\mu\left(a_{1}^{t_{n}}, a_{1}^{k}\right)-\mu\left(a_{1}^{t_{n}}\right) \mu\left(a_{1}^{k}\right)\right| \leq \beta\left(n-t_{n}-k\right) .
$$

Portanto, particularmente para $a_{1}^{t_{n}}$ conhecido,

$$
\left|\mu\left(a_{1}^{t_{n}}, a_{1}^{k}\right)-\mu\left(a_{1}^{t_{n}}\right) \mu\left(a_{1}^{k}\right)\right| \leq \beta\left(n-t_{n}-k\right),
$$

o que implica

$$
\begin{aligned}
\left|\mu\left(R_{n}(k) \cap X_{1}^{t_{n}}=a_{1}^{t_{n}}\right)-\mu\left(a_{1}^{t_{n}}\right) \mu\left(a_{1}^{k}\right)\right| & \leq\left|\mu\left(a_{1}^{t_{n}}, a_{1}^{k}\right)-\mu\left(a_{1}^{t_{n}}\right) \mu\left(a_{1}^{k}\right)\right| \\
& \leq \beta\left(n-t_{n}-k\right) .
\end{aligned}
$$

Ou seja, dividindo por $\mu\left(a_{1}^{t_{n}}\right)$

$$
\left|\frac{1}{\mu\left(a_{1}^{t_{n}}\right)} \mu\left(R_{n}(k) \cap X_{1}^{t_{n}}=a_{1}^{t_{n}}\right)-\mu\left(a_{1}^{k}\right)\right| \leq \frac{\beta\left(n-t_{n}-k\right)}{\mu\left(a_{1}^{t_{n}}\right)} .
$$

Conseguimos o primeiro limitante superior que procurávamos. Passemos agora à equação (2.84). Se $\omega \in R_{n}(i) \cap X_{1}^{t_{n}}=a_{1}^{t_{n}}$, então $\omega=a_{1}^{t_{n}} x_{t_{n}+1}^{n-t_{n}-i} a_{1}^{i}$, onde $a_{1}^{t_{n}}=a_{1}^{i} a_{i+1}^{t_{n}}$ e $\omega \notin$ 
$\left.\bigcup_{j=k}^{i-1} R_{n}(j)\right)$. Note que $\omega \notin \bigcup_{j=k}^{i-1} R_{n}(j)$ é equivalente a $a_{1}^{i} \notin \bigcup_{j=k}^{i-1} R_{i}(j)$. Logo,

$$
\begin{aligned}
\mu\left(R_{n}(i) \cap X_{1}^{t_{n}}=a_{1}^{t_{n}} \backslash \bigcup_{j=k}^{i-1} R_{n}(j)\right) & =\sum_{\omega \in R_{n}(i) \cap X_{1}^{t_{n}}=a_{1}^{t_{n}}} \mu\left(\omega: \omega \notin \bigcup_{j=k}^{i-1} R_{n}(j)\right) \\
& =\sum_{\omega \in R_{n}(i) \cap X_{1}^{t_{n}}=a_{1}^{t_{n}}} \mu\left(\omega: a_{1}^{i} \notin \bigcup_{j=k}^{i-1} R_{i}(j)\right) .
\end{aligned}
$$

Removendo o bloco $x_{t_{n}+1}^{n-t_{n}-i}$ de $\omega$ obteremos

$$
\mu\left(\omega: a_{1}^{i} \notin \bigcup_{j=k}^{i-1} R_{i}(j)\right) \leq \mu\left(a_{1}^{t_{n}}, a_{1}^{i}: a_{1}^{i} \notin \bigcup_{j=k}^{i-1} R_{i}(j)\right) .
$$

Mas, como $a_{1}^{t_{n}}$ é conhecido,

$$
\sum_{\omega \in R_{n}(i) \cap X_{1}^{t_{n}}=a_{1}^{t_{n}}} \mu\left(\omega: a_{1}^{i} \notin \bigcup_{j=k}^{i-1} R_{i}(j)\right) \leq \mu\left(a_{1}^{t_{n}}, a_{1}^{i}: a_{1}^{i} \notin \bigcup_{j=k}^{i-1} R_{i}(j)\right) .
$$

Pela propriedade $\beta$-mixing que o processo nos fornece,

$$
\sum_{a_{1}^{t_{n}, a_{1}^{i}}}\left|\mu\left(a_{1}^{t_{n}}, a_{1}^{i}\right)-\mu\left(a_{1}^{t_{n}}\right) \mu\left(a_{1}^{i}\right)\right| \leq \beta\left(n-t_{n}-i\right) .
$$

Portanto, particularmente para $a_{1}^{t_{n}}$ conhecido,

$$
\left|\mu\left(a_{1}^{t_{n}}, a_{1}^{i}: a_{1}^{i} \notin \bigcup_{j=k}^{i-1} R_{i}(j)\right)-\mu\left(a_{1}^{t_{n}}\right) \mu\left(a_{1}^{i} a_{1}^{i} \notin \bigcup_{j=k}^{i-1} R_{i}(j)\right)\right| \leq \beta\left(n-t_{n}-i\right),
$$

o que implica que

$$
\begin{aligned}
\mid \mu\left(R_{n}(i) \cap X_{1}^{t_{n}}=a_{1}^{t_{n}}\right) & -\mu\left(a_{1}^{t_{n}}\right) \mu\left(a_{1}^{i}: a_{1}^{i} \notin \bigcup_{j=k}^{i-1} R_{i}(j)\right) \mid \\
& \leq\left|\mu\left(a_{1}^{t_{n}}, a_{1}^{i}: a_{1}^{i} \notin \bigcup_{j=k}^{i-1} R_{i}(j)\right)-\mu\left(a_{1}^{t_{n}}\right) \mu\left(a_{1}^{i}: a_{1}^{i} \notin \bigcup_{j=k}^{i-1} R_{i}(j)\right)\right| \\
& \leq \beta\left(n-t_{n}-i\right) .
\end{aligned}
$$

Dividindo a expressão anterior por $\mu\left(a_{1}^{t_{n}}\right)$,

$$
\left|\frac{1}{\mu\left(a_{1}^{t_{n}}\right)} \mu\left(R_{n}(i) \cap X_{1}^{t_{n}}=a_{1}^{t_{n}}\right)-\mu\left(a_{1}^{i}: a_{1}^{i} \notin \bigcup_{j=k}^{i-1} R_{i}(j)\right)\right| \leq \frac{\beta\left(n-t_{n}-i\right)}{\mu\left(a_{1}^{t_{n}}\right)},
$$


e somando em $i$ desde $k+1$ até $t_{n}$, obtemos

$$
\begin{aligned}
\left|\sum_{i=k+1}^{t_{n}}\left(\frac{1}{\mu\left(a_{1}^{t_{n}}\right)} \mu\left(R_{n}(i) \cap X_{1}^{t_{n}}=a_{1}^{t_{n}}\right)-\mu\left(a_{1}^{i}: a_{1}^{i} \notin \bigcup_{j=k}^{i-1} R_{i}(j)\right)\right)\right| & \leq \frac{1}{\mu\left(a_{1}^{t_{n}}\right)} \sum_{i=k+1}^{t_{n}} \beta\left(n-t_{n}-i\right) \\
& <\frac{1}{\mu\left(a_{1}^{t_{n}}\right)} \sum_{i=n-2 t_{n}}^{n-t_{n}-k-1} \beta(i) .
\end{aligned}
$$

Tratemos agora a equação (2.85).

$$
\begin{aligned}
\left|\alpha_{k}-\alpha_{k, n}\right| & =\sum_{i=k+1}^{\infty} \mu\left(a_{1}^{i}: a_{1}^{i} \notin \bigcup_{j=k}^{i-1} R_{i}(j)\right)-\sum_{i=k+1}^{t_{n}} \mu\left(a_{1}^{i}: a_{1}^{i} \notin \bigcup_{j=k}^{i-1} R_{i}(j)\right) \\
& =\sum_{i=t_{n}+1}^{\infty} \mu\left(a_{1}^{i}: a_{1}^{i} \notin \bigcup_{j=k}^{i-1} R_{i}(j)\right) \\
& \leq \sum_{i=t_{n}+1}^{\infty} \mu\left(a_{1}^{i}\right) \\
& \leq \sum_{i=t_{n}+1}^{\infty} \inf _{1 \leq m<i}\left(\rho^{m}+\frac{\beta_{m}(i)}{2(1-\rho)}\right)
\end{aligned}
$$

onde a última linha foi obtida levando em consideração a desigualdade (2.15). Finalmente, limitaremos a equação (2.86). É claro que,

$$
\mu\left(\bigcup_{i=t_{n}+1}^{n-1} R_{i}(i) \cap X_{1}^{t_{n}}=a_{1}^{t_{n}}\right) \leq \mu\left(\bigcup_{i=t_{n}+1}^{n-1} R_{i}(i)\right) .
$$

Pela Relação de dualidade 1.18, temos que

$$
\mu\left(\bigcup_{i=t_{n}+1}^{n-1} R_{i}(i)\right)=\mu\left(\bigcup_{i=1}^{n-t_{n}} B_{n}(i)\right)
$$

Vamos escrever $c_{n} n=n-t_{n}$, onde $c_{n}=1-t_{n} / n$. Então, pelo Lema 1.16

$$
\mu\left(\bigcup_{i=1}^{c_{n} n} B_{n}(i)\right) \leq \mu\left(\bigcup_{i=\left\lfloor\frac{c_{n} n}{2}\right\rfloor}^{c_{n} n} B_{n}(i)\right) \leq \sum_{i=\left\lfloor\frac{c_{n} n}{2}\right\rfloor}^{c_{n} n} \mu\left(B_{n}(i)\right) .
$$

Como, $t_{n} \leq n / 2$ para $n$ fixo, $c_{n} \geq 1 / 2$, assim podemos usar a equação (2.33), obtendo

$$
\sum_{i=\left\lfloor\frac{c_{n} n}{2}\right\rfloor}^{c_{n} n} \mu\left(B_{n}(i)\right) \leq C_{1}(\rho) \sum_{i=c_{n}^{\prime}}^{n-t_{n}} \inf _{1 \leq m<i}\left(\rho^{m}+\tilde{\beta}_{m}(i)\right)
$$

onde $c_{n}^{\prime}=\min \left\{\left\lfloor t_{n} / 2\right\rfloor,\left\lfloor\left(n-t_{n}\right) / 2\right\rfloor\right\}$ e $C_{1}(\rho)$ é uma constante que só depende de $\rho$. Logo,

$$
\frac{1}{\mu\left(a_{1}^{t_{n}}\right)} \mu\left(\bigcup_{i=t_{n}+1}^{n-1} R_{i}(i) \cap X_{1}^{t_{n}}=a_{1}^{t_{n}}\right) \leq \frac{C_{1}(\rho)}{\mu\left(a_{1}^{t_{n}}\right)} \sum_{i=c_{n}^{\prime}}^{n-t_{n}} \inf _{1 \leq m<i}\left(\rho^{m}+\tilde{\beta}_{m}(i)\right) .
$$


Somando as inequações (2.87) e (2.88), obtemos que

$$
\begin{array}{r}
\left|\frac{1}{\mu\left(a_{1}^{t_{n}}\right)} \mu\left(R_{n}(k) \cap X_{1}^{t_{n}}=a_{1}^{t_{n}}\right)-\mu\left(a_{1}^{k}\right)\right|+\left|\sum_{i=k+1}^{t_{n}}\left(\frac{1}{\mu\left(a_{1}^{t_{n}}\right)} \mu\left(R_{n}(i) \cap X_{1}^{t_{n}}=a_{1}^{t_{n}}\right)-\mu\left(a_{1}^{i}: a_{1}^{i} \notin \bigcup_{j=k}^{i-1} R_{i}(j)\right)\right)\right| \\
\leq \frac{1}{\mu\left(a_{1}^{t_{n}}\right)} \sum_{i=n-2 t_{n}}^{n-t_{n}-k} \beta(i), \quad(2.92)
\end{array}
$$

e somando as inequações (2.89) e (2.91), levando em conta que $\beta_{m}(i)<\tilde{\beta}_{m}(i)$, chegamos a

$$
\left|\alpha_{k}-\alpha_{k, n}\right|+\frac{1}{\mu\left(a_{1}^{t_{n}}\right)} \mu\left(\bigcup_{i=t_{n}+1}^{n-1} R_{i}(i) \cap X_{1}^{t_{n}}=a_{1}^{t_{n}}\right) \leq \frac{C_{2}(\rho)}{\mu\left(a_{1}^{t_{n}}\right)} \sum_{i=c_{n}^{\prime}}^{\infty} \inf _{1 \leq m<i}\left(\rho^{m}+\tilde{\beta}_{m}(i)\right) .
$$

E como $\beta(i)<\tilde{\beta}(i) \leq \tilde{\beta}_{m}(i)$, pois $\beta$ é uma função decrescente, e somando as expressoẽs (2.92) e (2.93), temos que

$$
\begin{aligned}
\left|\mu\left(S_{n} \geq k \mid X_{1}^{t_{n}}=a_{1}^{t_{n}}\right)-\mu\left(a_{1}^{k}\right)-\alpha_{k}\right| & \frac{1}{\mu\left(a_{1}^{t_{n}}\right)} \sum_{i=n-2 t_{n}}^{n-t_{n}-k} \beta(i)+\frac{C_{2}(\rho)}{\mu\left(a_{1}^{t_{n}}\right)} \sum_{i=c_{n}^{\prime} n}^{\infty} \inf _{1 \leq m<i}\left(\rho^{m}+\tilde{\beta}_{m}(i)\right) \\
& \leq \frac{K_{1}(\rho)}{\mu\left(a_{1}^{t_{n}}\right)} \sum_{i=c_{n}^{\prime}}^{\infty} \inf _{1 \leq m<i}\left(\rho^{m}+\tilde{\beta}_{m}(i)\right),
\end{aligned}
$$

para uma constante $K_{1}(\rho)>1$, finalizando a primera parte do teorema. Para a convergência a zero, lembremos que $\mu\left(a_{1}^{t_{n}}\right)>0$ para todo $a_{1}^{t_{n}} \in \chi^{t_{n}}$, então existe $a>0 \in \mathbb{R}$ tal que $\mu\left(a_{1}^{t_{n}}\right) \geq a$, para todo $n$, o que implica que

$$
\frac{K_{1}(\rho)}{\mu\left(a_{1}^{t_{n}}\right)} \sum_{i=c_{n}^{\prime}}^{\infty} \inf _{1 \leq m<i}\left(\rho^{m}+\tilde{\beta}_{m}(i)\right) \leq \frac{K_{1}(\rho)}{a} \sum_{i=c_{n}^{\prime}}^{\infty} \inf _{1 \leq m<i}\left(\rho^{m}+\tilde{\beta}_{m}(i)\right) .
$$

Do mesmo modo que se provou que

$$
\lim _{n \rightarrow \infty} \sum_{i=\lfloor n / 8\rfloor}^{\infty} \inf _{1 \leq m<i}\left(\rho^{m}+\tilde{\beta}_{m}(i)\right)
$$

na convergência do Teorema 2.1 , tomando $m=\log _{\rho}(1 / f(i))$, onde $f(i)=i(\ln i)^{1+\epsilon}$, se prova que

$$
\lim _{n \rightarrow \infty} \sum_{i=c^{\prime} n}^{\infty} \inf _{1 \leq m<i}\left(\rho^{m}+\tilde{\beta}_{m}(i)\right)=0 .
$$

Assim, a convergência a zero que nós procuramos está garantida, pois $t_{n} / n$ diverge quando $n$ cresce infinitamente.

\subsubsection{Esperança condicional}

Nesta subseção vamos obter o limite e a velocidade de convergência da esperança de $S_{n}$ quando $n$ tende a infinito, dado que se conhece o processo até um tempo $t_{n}$. 
Teorema 2.16. Seja $\mu$ uma medida de probabilidade definida em $\chi^{\mathbb{N}}$ para um processo $\beta$ mixing com gramática completa, tal que $t_{n}<n / 2$ para todo $n$. Então,

$$
\left|\mathbb{E}\left(S_{n} \mid X_{1}^{t_{n}}=a_{1}^{t_{n}}\right)-\sum_{k=1}^{\infty} \mu\left(a_{1}^{k}\right)+\alpha_{k}\right| \leq \frac{K_{2}(\rho)}{\mu\left(a_{1}^{t_{n}}\right)} \sum_{i=c_{n}^{\prime}}^{\infty} i \inf _{1 \leq m<i}\left(\rho^{m}+\tilde{\beta}_{m}(i)\right),
$$

onde $K_{2}(\rho)$ é uma constante que só depende de $\rho, c_{n}^{\prime}=\min \left\{\left\lfloor t_{n} / 2\right\rfloor,\left\lfloor\left(n-t_{n}\right) / 2\right\rfloor\right\}$ e $\alpha_{k}$ como foi definida no Teorema 2.15. Se, além do anterior, o processo $\beta$-mixing é tal que $i \ln i \beta(i)$ é somável e $t_{n} / n$ tende a zero quando $n$ diverge, vale que

$$
\lim _{n \rightarrow \infty} \mathbb{E}\left(S_{n} \mid X_{1}^{t_{n}}=a_{1}^{t_{n}}\right)=\sum_{k=1}^{\infty}\left(\mu\left(a_{1}^{k}\right)+\alpha_{k}\right) .
$$

Demonstração. Pela definição de esperança condicional e pelo processo ter gramática completa, afirmamos que

$$
\mathbb{E}\left(S_{n} \mid X_{1}^{t_{n}}=a_{1}^{t_{n}}\right)=\sum_{k=1}^{n-1}\left(\mu\left(S_{n} \geq k \mid X_{1}^{t_{n}}=a_{1}^{t_{n}}\right)\right) .
$$

Dessa forma, podemos substituir a equação anterior na seguinte expressão:

$$
\begin{aligned}
\left|\mathbb{E}\left(S_{n} \mid X_{1}^{t_{n}}=a_{1}^{t_{n}}\right)-\sum_{k=1}^{n-1}\left(\mu\left(a_{1}^{k}\right)+\alpha_{k}\right)\right| \leq & \left|\sum_{k=1}^{t_{n}} \mu\left(S_{n} \geq k \mid X_{1}^{t_{n}}=a_{1}^{t_{n}}\right)-\sum_{k=1}^{t_{n}}\left(\mu\left(a_{1}^{k}\right)+\alpha_{k}\right)\right| \\
& +\left|\sum_{k=t_{n}+1}^{n-1} \mu\left(S_{n} \geq k \mid X_{1}^{t_{n}}=a_{1}^{t_{n}}\right)-\sum_{k=t_{n}+1}^{\infty} \mu\left(a_{1}^{k}\right)+\alpha_{k}\right| .
\end{aligned}
$$

Vamos aplicar o Teorema 2.15 na desigualdade (2.95), obtendo que

$$
\begin{aligned}
\left|\sum_{k=1}^{t_{n}} \mu\left(S_{n} \geq k \mid X_{1}^{t_{n}}=a_{1}^{t_{n}}\right)-\sum_{k=1}^{t_{n}}\left(\mu\left(a_{1}^{k}\right)+\alpha_{k}\right)\right| & \leq \sum_{k=1}^{t_{n}}\left|\mu\left(S_{n} \geq k \mid X_{1}^{t_{n}}=a_{1}^{t_{n}}\right)-\mu\left(a_{1}^{k}\right)-\alpha_{k}\right| \\
& \leq \frac{K_{1}(\rho)}{\mu\left(a_{1}^{t_{n}}\right)} \sum_{k=1}^{t_{n}} \sum_{i=c_{n}^{\prime}}^{\infty} \inf _{1 \leq m<i}\left(\rho^{m}+\tilde{\beta}_{m}(i)\right) \\
& =\frac{K_{1}(\rho) t_{n}}{\mu\left(a_{1}^{t_{n}}\right)} \sum_{i=c_{n}^{\prime}}^{\infty} \inf _{1 \leq m<i}\left(\rho^{m}+\tilde{\beta}_{m}(i)\right)
\end{aligned}
$$

onde $c_{n}^{\prime}=\min \left\{\left\lfloor t_{n} / 2\right\rfloor,\left\lfloor\left(n-t_{n}\right) / 2\right\rfloor\right\}$ e $K_{1}(\rho)$ é uma constante que só depende de $\rho$. Passemos agora à equação (2.96). É verdade que

$\left|\sum_{k=t_{n}+1}^{n-1} \mu\left(S_{n} \geq k \mid X_{1}^{t_{n}}=a_{1}^{t_{n}}\right)-\sum_{k=t_{n}+1}^{\infty} \mu\left(a_{1}^{k}\right)+\alpha_{k}\right| \leq \sum_{k=t_{n}+1}^{n-1} \mu\left(S_{n} \geq k \mid X_{1}^{t_{n}}=a_{1}^{t_{n}}\right)+\sum_{k=t_{n}+1}^{\infty} \mu\left(a_{1}^{k}\right)+\alpha_{k}$.

Vamos estudar separadamente cada equação à direita na desigualdade anterior. Começamos 
afirmando que, por definição de probabilidade condicional,

$$
\begin{aligned}
\mu\left(S_{n} \geq k \mid X_{1}^{t_{n}}=a_{1}^{t_{n}}\right) & \left.=\frac{1}{\mu\left(a_{1}^{t_{n}}\right)} \mu\left(S_{n} \geq k \cap X_{1}^{t_{n}}=a_{1}^{t_{n}}\right)\right) \\
& \leq \frac{1}{\mu\left(a_{1}^{t_{n}}\right)} \mu\left(S_{n} \geq k\right) \\
& =\frac{1}{\mu\left(a_{1}^{t_{n}}\right)} \mu\left(\bigcup_{i=k}^{n-1} R_{n}(i)\right),
\end{aligned}
$$

onde a última linha foi obtida graças à gramática completa do processo. Agora, usando a Relação de dualidade 1.18, chegamos a

$$
\begin{aligned}
\sum_{k=t_{n}+1}^{n-1} \mu\left(S_{n} \geq k \mid X_{1}^{t_{n}}=a_{1}^{t_{n}}\right) & =\frac{1}{\mu\left(a_{1}^{t_{n}}\right)} \sum_{k=t_{n}+1}^{n-1} \mu\left(\bigcup_{i=1}^{n-k} B_{n}(i)\right) \\
& \leq \frac{n-t_{n}}{\mu\left(a_{1}^{t_{n}}\right)} \mu\left(\bigcup_{i=1}^{n-t_{n}-1} B_{n}(i)\right) \\
& \leq \frac{\left(n-t_{n}\right) C_{1}(\rho)}{\mu\left(a_{1}^{t_{n}}\right)} \sum_{i=c_{n}^{\prime}}^{n-t_{n}} \inf _{1 \leq m<i}\left(\rho^{m}+\tilde{\beta}_{m}(i)\right),
\end{aligned}
$$

onde $C_{1}(\rho)$ é uma constante que só depende de $\rho$. Esta última linha foi obtida usando a inequação (2.90). Finalmente, vamos estudar o último somatório na desigualdade (2.98).

$$
\begin{aligned}
\sum_{k=t_{n}+1}^{\infty}\left(\mu\left(a_{1}^{k}\right)+\alpha_{k}\right) & \leq \sum_{k=t_{n}+1}^{\infty} \sum_{i=k}^{\infty} \mu\left(a_{1}^{i}\right) \\
& =\sum_{i=t_{n}+1}^{\infty} \mu\left(a_{1}^{i}\right)+\sum_{i=t_{n}+2}^{\infty} \mu\left(a_{1}^{i}\right)+\cdots+\sum_{i=n-1}^{\infty} \mu\left(a_{1}^{i}\right)+\ldots \\
& =\mu\left(a_{1}^{t_{n}+1}\right)+2 \mu\left(a_{1}^{t_{n}+2}\right)+\cdots+\left(n-t_{n}\right) \mu\left(a_{1}^{n}\right)+\ldots \\
& \leq \sum_{i=t_{n}+1}^{\infty} i \mu\left(a_{1}^{i}\right)
\end{aligned}
$$

Pela inequação (2.15), temos que

$$
\sum_{k=t_{n}+1}^{\infty}\left(\mu\left(a_{1}^{k}\right)+\alpha_{k}\right) \leq \sum_{i=t_{n}+1}^{\infty} i \inf _{1 \leq m<i}\left(\rho^{m}+\frac{\beta_{m}(i)}{2(1-\rho)}\right)
$$

Somando as desigualdades (2.97), (2.99) e (2.100) obtemos nosso resultado. Para a convergência a zero, lembremos que $\mu\left(a_{1}^{t_{n}}\right)>0$ para todo $a_{1}^{t_{n}} \in \chi^{t_{n}}$, então existe $a>0 \in \mathbb{R}$ tal que $\mu\left(a_{1}^{t_{n}}\right) \geq a$, para todo $n$, o que implica que

$$
\frac{K_{2}(\rho)}{\mu\left(a_{1}^{t_{n}}\right)} \sum_{i=b_{n}^{\prime}}^{\infty} \inf _{1 \leq m<i}\left(\rho^{m}+\tilde{\beta}_{m}(i)\right) \leq \frac{K_{2}(\rho)}{a} \sum_{i=b_{n}^{\prime}}^{\infty} \inf _{1 \leq m<i} i\left(\rho^{m}+\tilde{\beta}_{m}(i)\right) .
$$


Do mesmo modo que se provou que

$$
\lim _{n \rightarrow \infty} \sum_{i=\lfloor n / 8\rfloor}^{\infty} \inf _{1 \leq m<i} i\left(\rho^{m}+\tilde{\beta}_{m}(i)\right)
$$

na convergência do Teorema 2.10, tomando $m=\log _{\rho}(1 / f(i))$, onde $f(i)=i^{2}(\ln i)^{1+\epsilon}$, fica provado que

$$
\lim _{n \rightarrow \infty} \sum_{i=c^{\prime} n}^{\infty} \inf _{1 \leq m<i} i\left(\rho^{m}+\tilde{\beta}_{m}(i)\right)=0 .
$$

Assim, a convergência a zero que nos procuramos está garantida, pois $t_{n} / n$ diverge quando $n$ cresce infinitamente.

\subsubsection{Esperança da esperança condicional}

Agora vamos provar que a esperança do limite da esperança condicional é igual ao limite da esperança da esperança condicional, que como é sabido, coincide com o limite da esperança de $S_{n}$.

Teorema 2.17. Seja $\mu$ uma medida de probabilidade definida em $\chi^{\mathbb{N}}$ para um processo $\beta$ mixing com gramática completa tal que $i \ln i \beta(i)$ seja somável. Suponha que $t_{n}$ é uma função que satisfaz que $t_{n} / n \rightarrow 0$ quando $n \rightarrow \infty$. Então,

$$
\lim _{n \rightarrow \infty} \mathbb{E}\left(\mathbb{E}\left(S_{n} \mid X_{1}^{t_{n}}\right)\right)=\mathbb{E}\left(\lim _{n \rightarrow \infty} \mathbb{E}\left(S_{n} \mid X_{1}^{t_{n}}\right)\right) .
$$

Demonstração. Para provar este teorema vamos a usar o teorema da convergência dominada [24]. Pelo Teorema 2.16 na equação (2.94), $\mathbb{E}\left(S_{n} \mid X_{1}^{t_{n}}\right)$ converge puntualmente. Agora, pelo mesmo Teorema, temos que

$$
\begin{aligned}
\mathbb{E}\left(S_{n} \mid X_{1}^{t_{n}}=a_{1}^{t_{n}}\right) & \leq \sum_{i=1}^{n-1}\left(\mu\left(a_{1}^{i}\right)+\alpha_{i}\right)+\frac{K_{2}(\rho)}{\mu\left(a_{1}^{t_{n}}\right)} \sum_{i=b_{n}^{\prime}}^{\infty} i \inf _{1 \leq m<i}\left(\rho^{m}+\tilde{\beta}_{m}(i)\right) \\
& <\sum_{i=1}^{\infty}\left(\mu\left(a_{1}^{i}\right)+\alpha_{i}\right)+\frac{K_{2}(\rho)}{\mu\left(a_{1}^{t_{n}}\right)} \sum_{i=1}^{\infty} i \inf _{1 \leq m<i}\left(\rho^{m}+\tilde{\beta}_{m}(i)\right) .
\end{aligned}
$$

Analisaremos separadamente cada um dos somatórios anteriores. Como foi ilustrado na prova do Teorema 2.16,

$$
\sum_{i=1}^{\infty}\left(\mu\left(a_{1}^{i}\right)+\alpha_{i}\right) \leq \sum_{i=1}^{\infty} i \mu\left(a_{1}^{i}\right) \leq \sum_{i=1}^{\infty} i \inf _{1 \leq m<i}\left(\rho^{m}+\frac{\beta_{m}(i)}{2(1-\rho)}\right)
$$

onde a última desigualdade foi obtida pela expressão (2.15). Logo,

$$
\mathbb{E}\left(S_{n} \mid X_{1}^{t_{n}}=a_{1}^{t_{n}}\right)<\frac{K_{3}(\rho)}{\mu\left(a_{1}^{t_{n}}\right)} \sum_{i=1}^{\infty} i \inf _{1 \leq m<i}\left(\rho^{m}+\tilde{\beta}_{m}(i)\right) .
$$


Se tomarmos $m=\log _{\rho}(1 / f(i))$, onde $f(i)=i^{2}(\ln i)^{1+\epsilon}$ para um $\epsilon$ entre 0 e 1 , temos que

$$
\mathbb{E}\left(S_{n} \mid X_{1}^{t_{n}}=a_{1}^{t_{n}}\right)<\frac{K_{3}(\rho)}{\mu\left(a_{1}^{t_{n}}\right)} \sum_{i=1}^{\infty}\left(\frac{1}{i(\ln i)^{\epsilon}}+i \tilde{\beta}\left(\left\lfloor\frac{-i \ln \rho}{2 i+(1+\epsilon) \ln i}\right\rfloor-1\right)\right) .
$$

Como $i(\ln i)^{1+\epsilon}$ é somável, existe um número real $M_{1}$, tal que

$$
\sum_{i=1}^{\infty} \frac{1}{i(\ln i)^{\epsilon}}=M_{1}
$$

Por outro lado, como foi mostrado na prova do Teorema 2.10 na desigualdade (2.66),

$$
\sum_{i=1}^{\infty} i \tilde{\beta}\left(\left\lfloor\frac{-i \ln \rho}{2 i+(1+\epsilon) \ln i}\right\rfloor-1\right) \leq \frac{3}{\rho_{1}} \sum_{i=1}^{\infty} i \ln (i+1) \beta(i),
$$

onde $\rho_{1}=-\ln \rho / 3$. Por hipótese $i \ln i \beta(i)$ é somável, portanto, existe um número real $M_{2}(\rho)$, que depende de $\rho$, tal que

$$
\sum_{i=1}^{\infty} i \beta\left(\left\lfloor\frac{i}{\log _{\rho}(1 / f(i))}\right\rfloor-1\right)=M_{2}(\rho) .
$$

Das desigualdades (2.104) e (2.105), obtemos que

$$
\mathbb{E}\left(S_{n} \mid X_{1}^{t_{n}}=a_{1}^{t_{n}}\right)<M_{1}+M_{2}(\rho)=M(\rho) .
$$

Dado que $\mathbb{E}(M(\rho))=M(\rho)$ porque $M(\rho) \in \mathbb{R}$ e pelo teorema da convergência dominada, obtemos nosso resultado.

\subsection{Entropia vs $\mathbb{E}\left(S_{n}\right)$}

Na subseção 1.2.3, foi conjeturado que existia uma relação entre a entropia do processo independente e identicamente distribuído e a esperança da variável $S_{n}$. Miguel Abadi, Rodrigo Lambert \& Guilherme Ludwig simularam esta relação para uma cadeia de Markov no conjunto de estados $\{0,1\}$ com matriz probabilidades de transição

$$
P=\frac{0}{1}\left(\begin{array}{cc}
\mathrm{p} & 1 \\
1-\mathrm{q} & \mathrm{q}
\end{array}\right)
$$

onde $0<p, q<1$. Eles simularam palavras de tamanho 25 variando $p$ e $q$ com malha 0.05 . Os gráficos da Esperança de $S_{n}$ e da Entropia são mostrados nas figuras 2.5 e 2.6. 


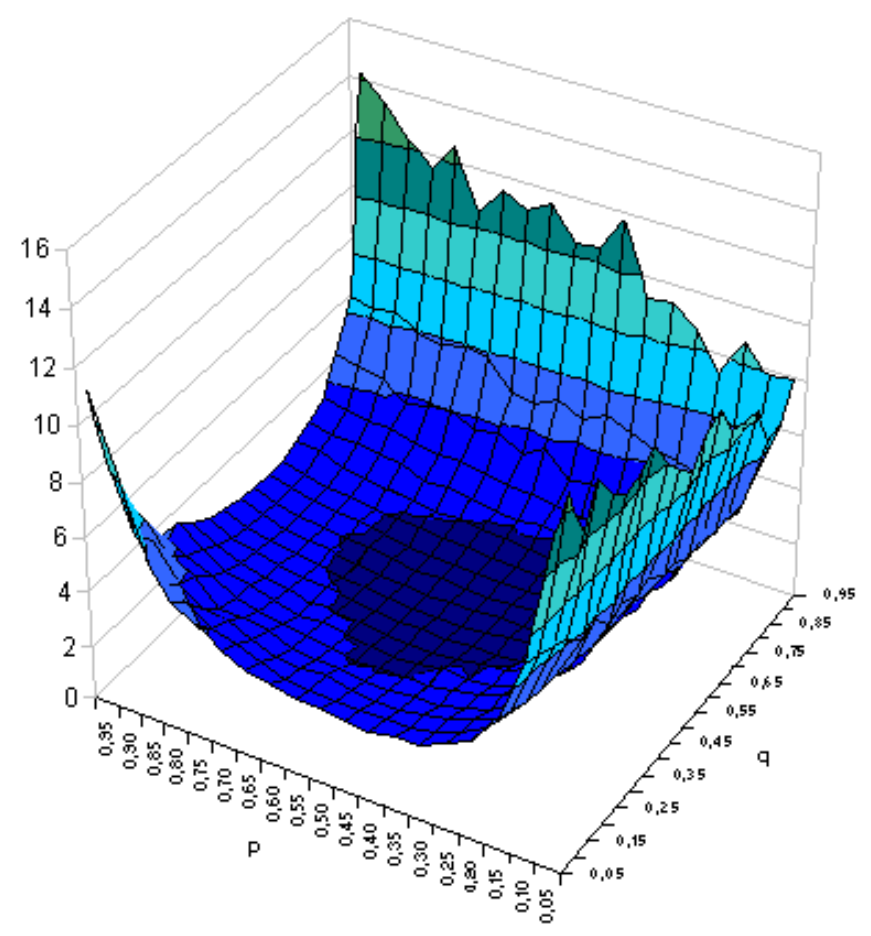

Figura 2.5: $E\left(S_{n}\right)$ para o processo de Markov com gramática completa.

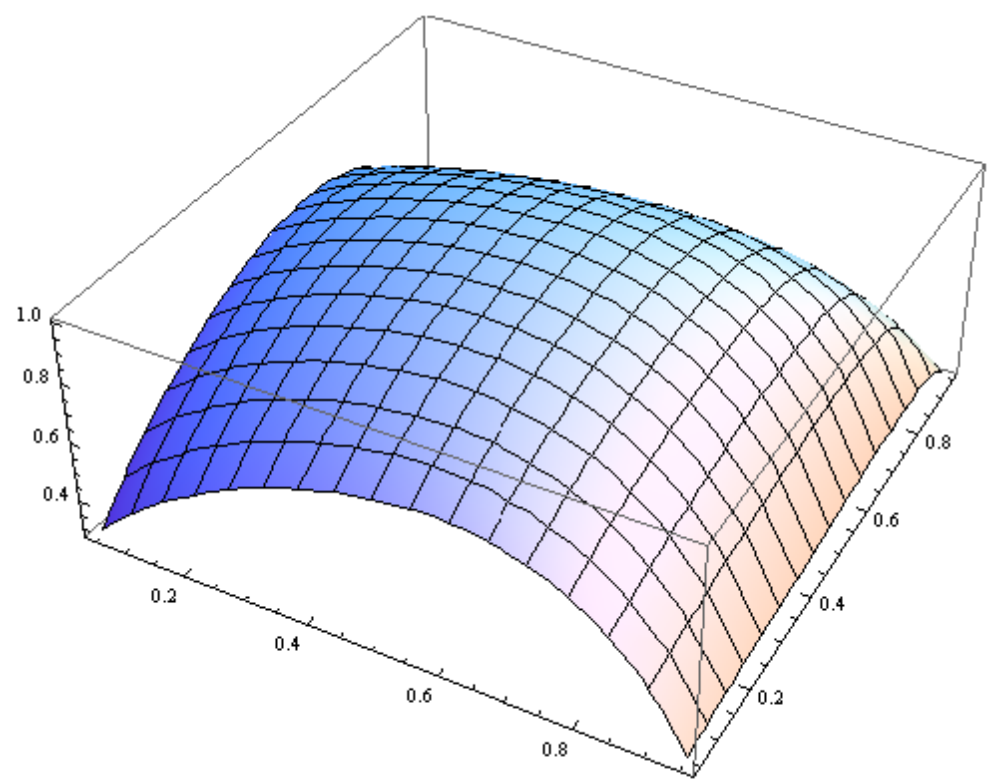

Figura 2.6: Entropia para o processo de Markov com gramática completa.

Pode-se conjeturar, como no caso independente, que poderia existir uma relação inversa entre as quantidades entropia e esperança de $S_{n}$ para um processo de Markov.

Tanto como o processo independente quanto a cadeia de Markov simulada anteriormente têm gramática completa e são processos $\beta$-mixing. Quisemos comprovar se esta suposta relação inversa se mantém para a seguinte cadeia de Markov com gramática completa. Esta cadeia assume valores no conjunto $\chi=\{0,1,2\}$ e tem as seguintes probabilidades de transição: 


$$
P=\begin{gathered}
0 \\
1 \\
2
\end{gathered}\left(\begin{array}{ccc}
1-q_{0} & q_{0} / 2 & 2 \\
\left(1-q_{1}\right) / 2 & q_{1} / 2 & \left(1-q_{1}\right) / 2 \\
1 / 3 & 1 / 3 & 1 / 3
\end{array}\right)
$$

onde $0<q_{i}<1$, para $i=0,1$.

Passemos agora a simular o contraste entre a $E\left(S_{n}\right)$ e a entropia do processo. Nós simulamos palavras de tamanho 8, para $q_{0}$ e $q_{1}$ variando entre 0.01 e 0.99 com malha de 0.05 . Os gráficos da esperança de $S_{n}$ e da entropia são mostrados nas figuras seguintes:

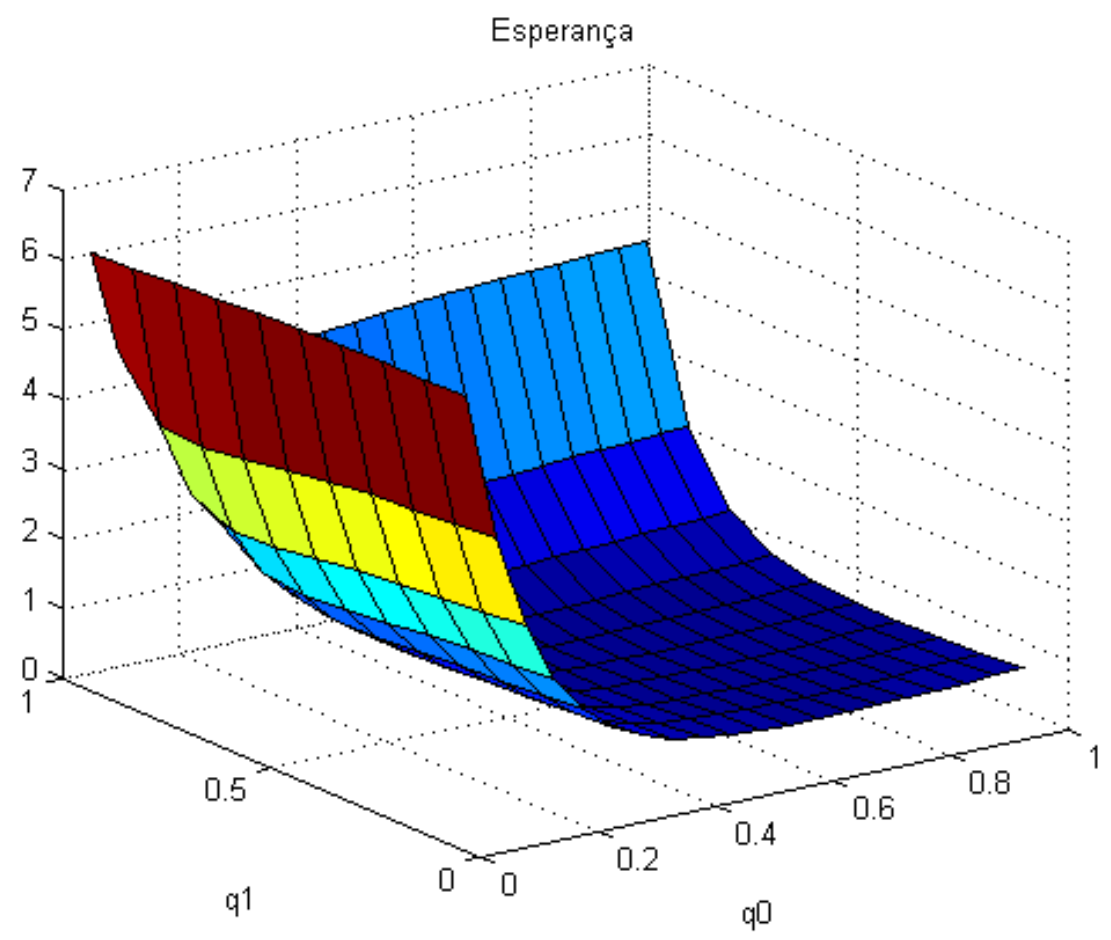

Figura 2.7: $E\left(S_{n}\right)$ para o processo de Markov com gramática completa 


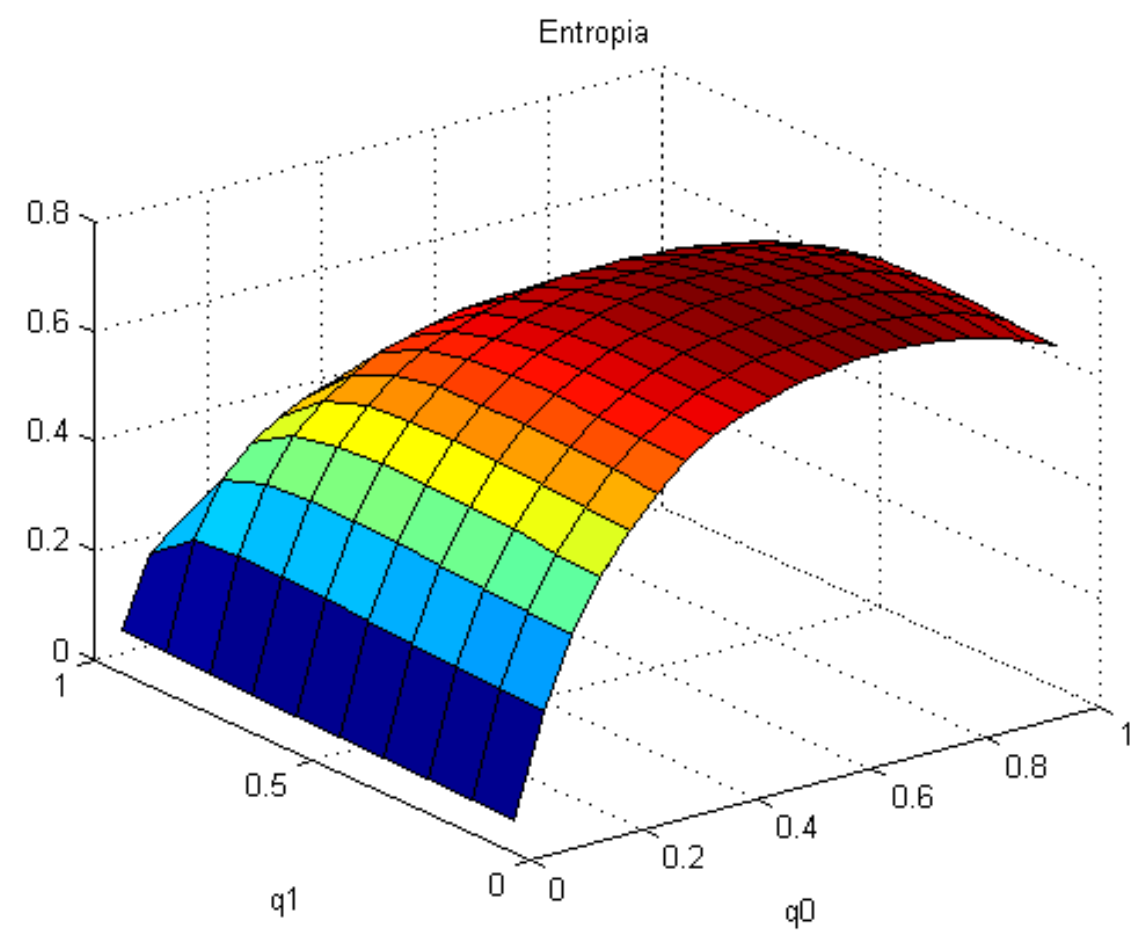

Figura 2.8: Entropia para o processo de Markov com gramática completa

\subsection{Caso independente}

Nesta seção vamos apresentar limitantes mais eficientes para o limite da esperança de $S_{n}$ que os dados no Corolário 1.27. E como novidade também apresentamos limitantes para o limite do segundo momento finito e, consequentemente, para o limite da variância.

Teorema 2.18. Seja $\mu$ a medida produto sobre $\chi^{\mathbb{N}}$ com marginais identicamente distribuidas. Considere $Q=\lim _{n \rightarrow \infty} \mathbb{E}\left(S_{n}\right)$. Então,

$$
\max \left\{\frac{m_{2}}{1-m_{2}}, \frac{m_{2}}{\left(1-m_{2}\right)^{2}}-\frac{m_{4}}{\left(1-m_{4}\right)^{2}}\left(\frac{1+m_{2}}{1-m_{2}}\right)\right\} \leq Q \leq \frac{m_{2}}{\left(1-m_{2}\right)^{2}}-\frac{m_{4}}{\left(1-m_{4}\right)\left(1-m_{2}\right)}
$$

Demonstração. Nós temos que,

$$
Q=\sum_{k=1}^{\infty} \lim _{n \rightarrow \infty} \mu\left(S_{n} \geq k\right)=\sum_{k=1}^{\infty} m_{2}^{k}+a_{k}
$$

Pela Proposição 1.22

$$
\begin{aligned}
Q & \leq \frac{m_{2}}{1-m_{2}}+\sum_{k=1}^{\infty} \frac{m_{2}^{k+1}}{1-m_{2}}-\frac{m_{4}^{k}}{1-m_{2}} \\
& \leq \frac{m_{2}}{1-m_{2}}+\frac{m_{2}^{2}}{\left(1-m_{2}\right)^{2}}-\frac{m_{4}}{\left(1-m_{4}\right)\left(1-m_{2}\right)} \\
& \leq \frac{m_{2}}{\left(1-m_{2}\right)^{2}}-\frac{m_{4}}{\left(1-m_{4}\right)\left(1-m_{2}\right)} .
\end{aligned}
$$


No outro sentido da desigualdade, pela Proposição 1.23, obtemos que

$$
\begin{aligned}
Q & \geq \frac{m_{2}}{1-m_{2}}+\sum_{k=1}^{\infty} \frac{m_{2}^{k}}{1-m_{2}}-\frac{m_{4}^{k}}{1-m_{2}}\left(\frac{1+m_{2}}{1-m_{4}}\right) \\
& \geq \frac{m_{2}}{1-m_{2}}+\frac{m_{2}^{2}}{\left(1-m_{2}\right)^{2}}-\frac{m_{4}}{\left(1-m_{4}\right)^{2}}\left(\frac{1+m_{2}}{1-m_{2}}\right) .
\end{aligned}
$$

Para mostrar um exemplo gráfico do comportamento do limite da esperança de $S_{n}$, seja $\chi=\{0,1\}, p=\mu(1)$ e $1-p=\mu(0)$. Na figura 2.9 , temos que

$$
\begin{aligned}
\mathrm{A} & =\frac{m_{2}}{1-m_{2}}, \\
\mathrm{~B} & =\frac{m_{2}}{\left(1-m_{2}\right)^{2}}-\frac{m_{4}}{\left(1-m_{4}\right)^{2}}\left(\frac{1+m_{2}}{1-m_{2}}\right) \\
\mathrm{C} & =\frac{m_{2}}{\left(1-m_{2}\right)^{2}}-\frac{m_{4}}{\left(1-m_{4}\right)\left(1-m_{2}\right)} .
\end{aligned}
$$

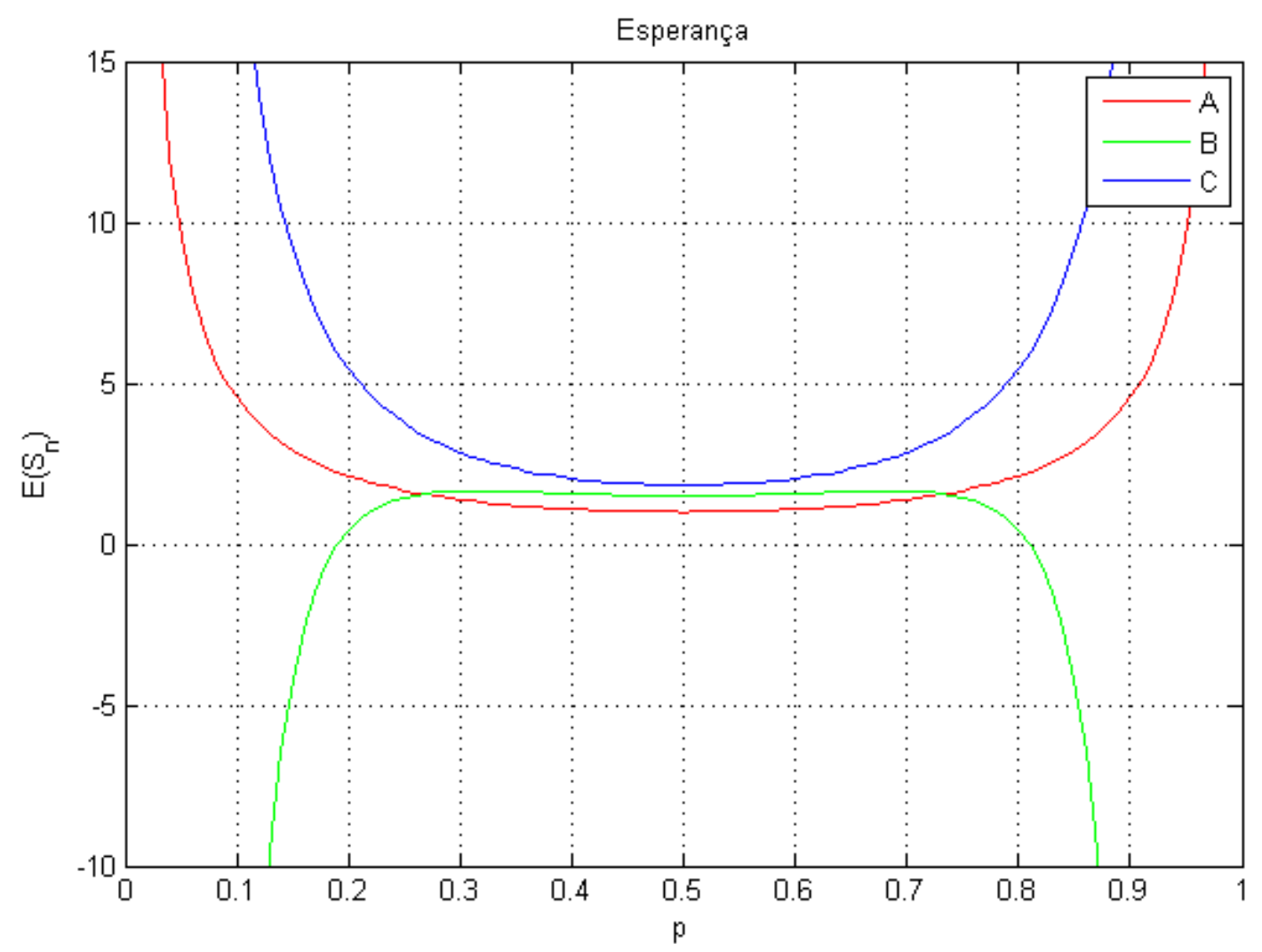

Figura 2.9: Limitantes superior e inferior para o limite de $E\left(S_{n}\right)$.

Portanto, o limite da esperança de $S_{n}$ se encontra entre a linha azul e o máximo entre as linhas verde e vermelha. Os limitantes dados funcionan razoavelmente bem para $0.3<p<0.7$. No caso em que $p=0.5,1.5102 \leq \mathbb{E}\left(S_{n}\right) \leq 1.8367$. 
O próximo teorema apresenta limitantes para o limite do segundo momento finito de $S_{n}$.

Teorema 2.19. Sejam $\chi$ um alfabeto e $\mu$ a medida produto sobre $\chi^{\mathbb{N}}$ com marginais identicamente distribuidas. Considere $Q_{2}=\lim _{n \rightarrow \infty} \mathbb{E}\left(S_{n}^{2}\right)$. Então,

$$
\max \{A, B\} \leq Q_{2} \leq \frac{m_{2}+m_{2}^{2}}{\left(1-m_{2}\right)^{3}}-\frac{m_{4}}{\left(1-m_{4}\right)^{2}},
$$

onde

$$
A=\frac{m_{2}}{1-m_{2}}\left(\frac{2}{1-m_{2}}-1\right), \quad e \quad B=\frac{m_{2}+m_{2}^{2}}{\left(1-m_{2}\right)^{3}}-\frac{2 m_{4}}{\left(1-m_{4}\right)^{3}}\left(\frac{1+m_{2}}{\left(1-m_{2}\right)}-\frac{1}{2}\right) .
$$

Demonstração. Nós temos que

$$
\begin{aligned}
Q_{2} & =\sum_{k=1}^{\infty}(2 k-1) \lim _{n \rightarrow \infty} \mu\left(S_{n} \geq k\right)=\sum_{k=1}^{\infty}(2 k-1) m_{2}^{k}+a_{k} \\
& =2 \sum_{k=1}^{\infty} k\left(m_{2}^{k}+a_{k}\right)-\sum_{k=1}^{\infty} m_{2}^{k}+a_{k}
\end{aligned}
$$

Vamos calcular o primeiro termo da igualdade anterior, pois o segundo termo é dado pelo Teorema 2.18. Aplicando de novo a Proposição 1.22, fica que

$$
2 \sum_{k=1}^{\infty} k m_{2}^{k}+\sum_{k=1}^{\infty} k a_{k} \leq \frac{2 m_{2}}{\left(1-m_{2}^{2}\right)}+\frac{2}{1-m_{2}}\left(\frac{m_{2}^{2}}{\left(1-m_{2}^{2}\right)}-\frac{m_{4}}{\left(1-m_{4}^{2}\right)}\right) .
$$

Logo, aplicando o Teorema 2.18 e simplificando a expressão, o resultado é finalmente a primeira desigualdade

$$
Q_{2} \leq \frac{m_{2}+m_{2}^{2}}{\left(1-m_{2}\right)^{3}}-\frac{m_{4}}{\left(1-m_{4}\right)^{2}}
$$

No outro sentido da desigualdade, aplicando a Proposição 1.23, teremos

$$
2 \sum_{k=1}^{\infty} k m_{2}^{k}+\sum_{k=1}^{\infty} k a_{k} \geq \frac{2 m_{2}}{\left(1-m_{2}\right)^{2}}+\frac{2 m_{2}^{2}}{\left(1-m_{2}\right)^{3}}-\frac{2 m_{4}}{1-m_{2}}\left(\frac{1+m_{2}}{\left(1-m_{4}\right)^{2}}\right) .
$$

Aplicando o Teorema 2.18 e simplificando a expressão, chegamos finalmente a desigualdade

$$
Q_{2} \geq \frac{m_{2}+m_{2}^{2}}{\left(1-m_{2}\right)^{3}}-\frac{2 m_{4}}{\left(1-m_{4}\right)^{3}}\left(\frac{1+m_{2}}{\left(1-m_{2}\right)}-\frac{1}{2}\right)
$$

O limite inferior mostrado na última inequação não funciona muito bem em certos valores $p$, por isso, procuramos mais um limitante inferior e escolheremos o valor máximo entre o dois. O limitante calculado é baseado no Corolário 1.27, assim

$$
\begin{aligned}
Q_{2} & \geq \sum_{k=1}^{\infty} k m_{2}^{k}-\sum_{k=1}^{\infty} m_{2}^{k} \\
& =\frac{m_{2}}{\left(1-m_{2}\right)^{2}}-\frac{m_{2}}{1-m_{2}} \\
& =\frac{m_{2}}{1-m_{2}}\left(\frac{2}{1-m_{2}}-1\right),
\end{aligned}
$$


chegando ao resultado desejado.

Continuando com nosso exemplo com distribuição Bernoulli, nós graficamos os limitantes para $Q_{2}$ usando a letra $C$ que representa o limite superior na equação (2.107). Na figura 2.10, usamos

$$
\begin{aligned}
\mathrm{A} & =\frac{m_{2}}{1-m_{2}}\left(\frac{2}{1-m_{2}}-1\right), \\
\mathrm{B} & =\frac{m_{2}+m_{2}^{2}}{\left(1-m_{2}\right)^{3}}-\frac{2 m_{4}}{\left(1-m_{4}\right)^{3}}\left(\frac{1+m_{2}}{\left(1-m_{2}\right)}-\frac{1}{2}\right), \\
\mathrm{C} & =\frac{m_{2}+m_{2}^{2}}{\left(1-m_{2}\right)^{3}}-\frac{m_{4}}{\left(1-m_{4}\right)^{2}} .
\end{aligned}
$$

O limite do segundo momento finito de $S_{n}$ se encontra entre a linha azul e o máximo entre

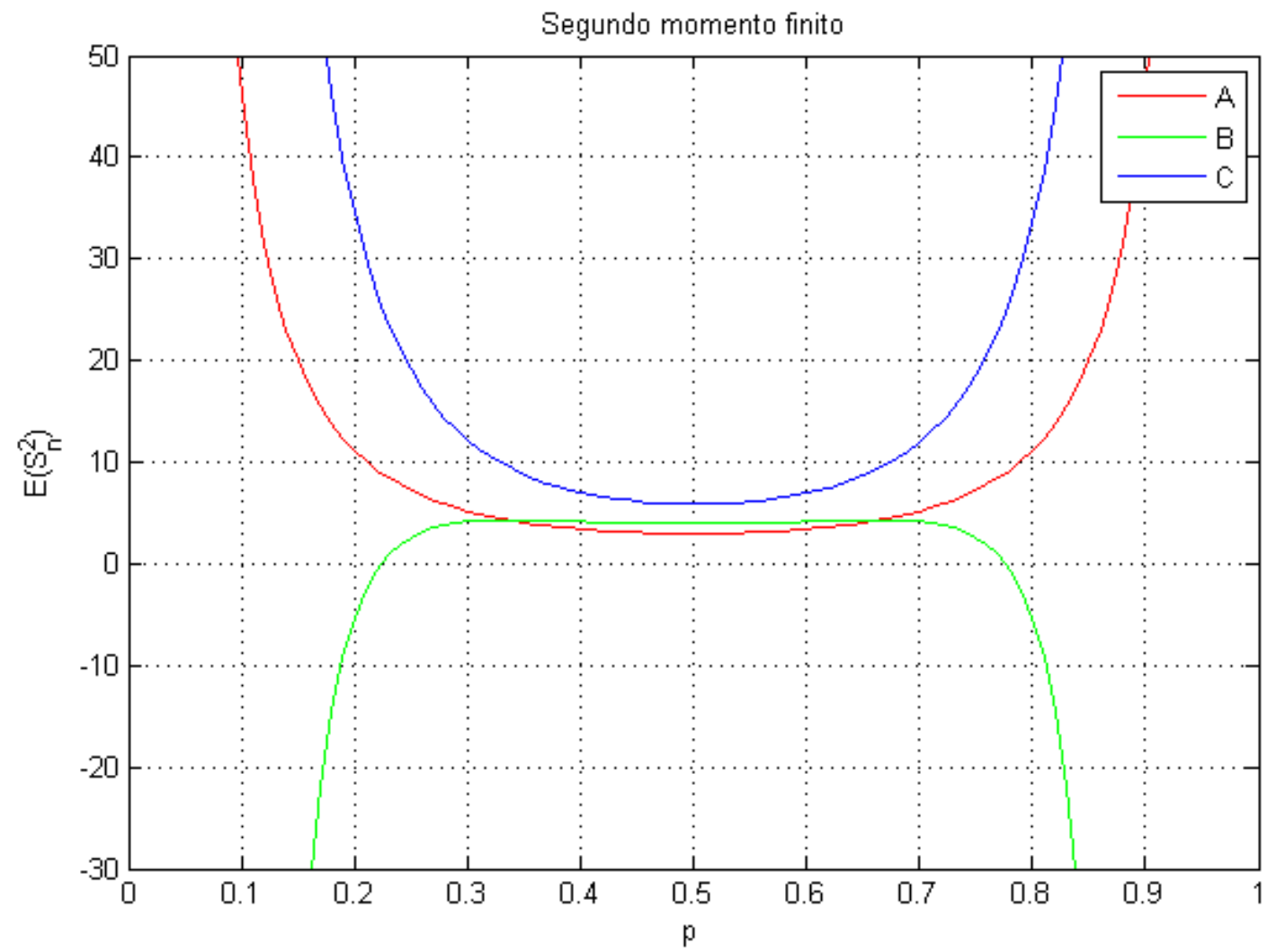

Figura 2.10: Limitantes superior e inferior para o limite do segundo momento de $E\left(S_{n}\right)$.

as linhas verde e vermelha da figura 2.10, onde se vê que os limitantes funcionam muito bem para $0.4<p<0.6$. No caso em que $p=0.5,3.9475 \leq \mathbb{E}\left(S_{n}^{2}\right) \leq 5.8750$.

levando em conta os Teoremas 2.18 e 2.19 apresentamos os limitantes para o limite da Variância de $S_{n}$ no seguinte teorema.

Teorema 2.20. Sejam $\chi$ um alfabeto e $\mu$ a medida produto sobre $\chi^{\mathbb{N}}$ com marginais identi- 
camente distribuidas. Considere $\operatorname{Var} Q=\lim _{n \rightarrow \infty} \operatorname{Var}\left(S_{n}\right)$. Então,

$\max \{a, b\} \leq \operatorname{Var} Q \leq \frac{m_{2}-m_{2}^{2}-m_{2}^{3}}{\left(1-m_{2}\right)^{4}}-\frac{m_{4}}{\left(1-m_{4}\right)}\left(\frac{1}{1-m_{4}}+\frac{m_{4}}{\left(1-m_{2}\right)\left(1-m_{4}\right)}-\frac{2 m_{2}}{\left(1-m_{2}\right)^{3}}\right)$,

onde

$$
\begin{aligned}
& a=\frac{m_{2}}{1-m_{2}}\left(\frac{2}{1-m_{2}}-\frac{m_{2}}{\left(1-m_{2}\right)^{3}}-1\right)-\frac{m_{4}}{\left(1-m_{2}\right)^{2}\left(1-m_{4}\right)}\left(\frac{m_{4}}{1-m_{4}}-\frac{2 m_{2}}{1-m_{2}}\right), \quad e \\
& b=\frac{m_{2}+m_{2}^{2}}{\left(1-m_{2}\right)^{4}}-\frac{2 m_{4}}{\left(1-m_{2}\right)\left(1-m_{4}\right)}\left(\frac{1+m_{2}}{\left(1-m_{4}\right)^{2}}+\frac{m_{4}}{2\left(1-m_{2}\right)\left(1-m_{4}\right)}-\frac{m_{2}}{\left(1-m_{2}\right)^{2}}-\frac{1}{2}\right) .
\end{aligned}
$$

Demonstração. A prova é imediata aplicando os Teoremas 2.18 e 2.19 e simplificando as expressoẽs resultantes.

No gráfico para o exemplo Bernoulli, a letra $c$ representa o limite superior da $\operatorname{Var} Q$ do Teorema 2.20. O limite da variância de $S_{n}$ se encontra entre a linha azul e o máximo entre as linhas verde e vermelha da figura 2.11. Lembramos que neste gráfico

$$
\begin{aligned}
\mathrm{a} & =\frac{m_{2}}{1-m_{2}}\left(\frac{2}{1-m_{2}}-\frac{m_{2}}{\left(1-m_{2}\right)^{3}}-1\right)-\frac{m_{4}}{\left(1-m_{2}\right)^{2}\left(1-m_{4}\right)}\left(\frac{m_{4}}{1-m_{4}}-\frac{2 m_{2}}{1-m_{2}}\right), \\
\mathrm{b} & =\frac{m_{2}+m_{2}^{2}}{\left(1-m_{2}\right)^{4}}-\frac{2 m_{4}}{\left(1-m_{2}\right)\left(1-m_{4}\right)}\left(\frac{1+m_{2}}{\left(1-m_{4}\right)^{2}}+\frac{m_{4}}{2\left(1-m_{2}\right)\left(1-m_{4}\right)}-\frac{m_{2}}{\left(1-m_{2}\right)^{2}}-\frac{1}{2}\right), \\
\mathrm{c} & =\frac{m_{2}-m_{2}^{2}-m_{2}^{3}}{\left(1-m_{2}\right)^{4}}-\frac{m_{4}}{\left(1-m_{4}\right)}\left(\frac{1}{1-m_{4}}+\frac{m_{4}}{\left(1-m_{2}\right)\left(1-m_{4}\right)}-\frac{2 m_{2}}{\left(1-m_{2}\right)^{3}}\right) .
\end{aligned}
$$

Aqui, os limitantes funcionam muito bem para $0.4<p<0.6$. No caso em que $p=0.5$, $2.2274 \leq \operatorname{Var}\left(S_{n}\right) \leq 2.9388$. 


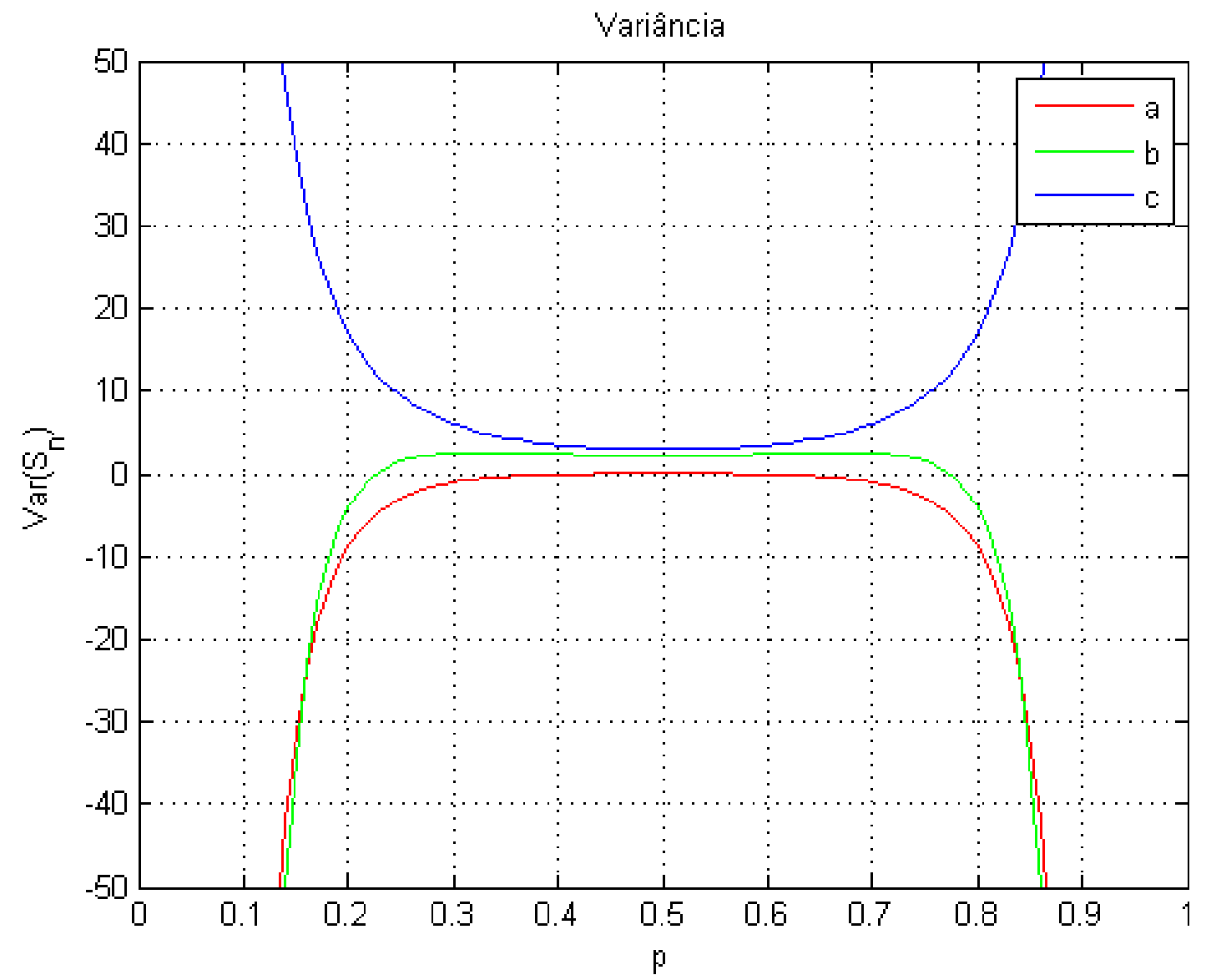

Figura 2.11: Limitantes superior e inferior para o limite da Variância de $E\left(S_{n}\right)$. 


\section{Capítulo 3}

\section{Conclusões e Problemas em aberto}

Este trabalho teve como objetivo fundamental generalizar o trabalho feito por Lambert [19] e Abadi e Lambert [5]. Eles essencialmente provaram que existe a distribuição limite da função $S_{n}$ quando o processo que gera as variáveis é independente e identicamente distribuído. Além disso, mostraram o limite explicitamente. Nossa generalização foi feita para processos ergódicos com gramática completa, mas trocando a hipótese de independência por uma hipótese de dependência $\beta$-mixing. Chegamos a conclusão da existência da distribuição limite da função $S_{n}$, da $\mathbb{E}\left(S_{n}\right)$ e da $\operatorname{Var}\left(S_{n}\right)$. Além disso, obtivemos resultados condicionais.

Uma questão a responder é se os resultados obtidos nesta tese, são válidos para processos sem gramática completa nos quais $\mu\left(S_{n}<0\right)>0$.

Outra pergunta interessante, que ainda permanece sem resposta, é a relação que apresentam a entropia, $h$, do processo e a esperança de $S_{n}$. Será possível achar uma relação entre estas duas quantidades de tal forma que se possa calcular $h$ por medio de $E\left(S_{n}\right)$ ?. 


\section{Apêndice A}

\section{Artigo}

Durante o doutorado, eu fiz uma visita á Universidade do Sur da Califórnia (University of Southern California) para trabalhar sob a orientação do professor Nicolai Haydn. Nós, junto a seus alunos Chimanya Gupta (estudante de pós-doutorado) e Milton Ko (estudante de doutorado), discutimos temas relacionado à teoria ergódica. Essas discussões tiveram como resultado um artigo em conjunto, o qual pode-se encontrar no arxiv.org com o seguinte link arXiv:1306.4476. O título do artigo e o resumo, ambos em português, são:

\section{Título: Tempos de entrada e Entropia de Rényi}

\section{Resumo:}

Para sistemas ergódicos com partições geradoras, o resultado bem conhecido de Ornstein e Weiss mostra que a tasa de crescimento exponencial do tempo de recorrência é igual à entropia métrica quase certamente. Aqui nós estudamos a tasa de crescimento exponencial do tempo de entrada, e mostramos que ele é igual à entropia, onde a convergência é em probabilidade e usando a medida produto. Mas precisamos como hipótese que a distribuição limite dos tempos de entrada exista quase certamente. Essa condição parece natural, sob a óptica de um exemplo feito por Shields no qual o limsup da tasa de crescimento exponencial é infinito quase certamente excepto onde os tempos de entrada não existem. Nosotros consideramos também sistemas $\phi$-mixing e provamos um resultado que conecta a entropia de Rényi com os tempos de entrada. 
60 APÊNDICE A 


\section{Referências Bibliográficas}

[1] M. Abadi, Exponential approximation for hitting times in mixing processes, Mathematical Physics Electronis Journal 7 (2001), no. 2. xiii, xiv, 3

[2] _ Sharp error terms and neccessary conditions for exponential hitting times in mixing processes, The Annals of Probability 32 (2004), no. 1A, 243-264. 3, 13

[3] _ Poisson approximations via chen-stein for non-markov processes., In and out of equilibrium. 2. Progress in Probability 60 (2008), no. 2, 1-19. xiii

[4] M. Abadi and L. Cardeño, Renyi entropies and large deviation for the overlapping function. xiv

[5] M. Abadi and R. Lambert, The distribution of the short-return function., Nonlinearity 26 (2013), no. 5, 1143-1162. v, vii, xv, 4, 6, 9, 57

[6] M. Abadi and B. Saussol, Hitting and returning into rare events for all alpha-mixing processes, Stochastic process and theirs applications 121 (2010), no. 2, 314-323. 3

[7] M. Abadi and S. Vaienti, Large deviations for short recurrence, Discrete and Continuous Dynamical Systems - Series A (DCDS-A) 21 (2008), no. 3, 729 - 747. xiii, xiv, 6

[8] Liliam Cardeño Acero, Comportamentos globais e locais de tempos de entrada curtos, Ph.D. thesis, Universidade de São Paulo, Novembro 2007. 6

[9] V. Afraimovich, J. Chazottes, and B. Saussol, Pointwise dimensions for poincaré recurrence associated with maps and special flows, Discrete and Continuous Dynamical Systems 9 (2003), 263-280. xiv, 7

[10] H. Berbee, Convergence rates in the strong law for bounded mixing sequences, Probab. Th. Rel. Fields 74 (1987), 255-270. 27

[11] R. Bradley, Basic properties of strong mixing conditions. a survey and some open questions, Probability Surveys 2 (2005), 107-144. 1, 2, 14

[12] Roberto Fernández, Pablo Ferrari, and Antonio Galves, Coupling, renewal and perfect simulation of chains of infinite order, notas para o mini-curso presentado na vth escola brasileira de probabilidade ed., http://www.ime.usp.br/ pablo/abstracts/vebp.html, 2001. 26

[13] A. Galves, P. Collet, and B. Schmitt, Repetition times for gibbsian sourses, Nonlinearity 12 (1999), 1225-1237. xiv

[14] A. Galves and B. Schmitt, Inequalities for hitting times in mixing dynamical systems, Random Comput. Dyn 5 (1997), 337-348. 3, 13 
[15] N. Haydn and S. Vaienti, The compound poisson distribution and return times in dynamical systems, Prob. Theory Related Fields 144 (2009), no. 1, 159-179. xv

[16] _ The renyi entropy function and the large deviation of short return times, Ergodic Theory and Dynamical Systems 30 (2010), 159-179. xiii

[17] M. Hirata, Poisson law for axiom a diffeomorphisms, Ergodic Theory and Dynamical Systems 13 (1993), no. 3, 533-556. xiii

[18] M. Hirata, B. Saussol, and S. Vaienti, Statistics of returns times: a general framework and new applications., Comm. Math. Phys. 206 (1999), no. 3, 33-55. xiv

[19] Rodrigo Lambert, Comportamento assentótico do primeiro retorno de uma sequência gerada por variáveis aleátorias independentes e identicamente distribuídas., Master's thesis, Instituto de Matemática, Estatística e Computção, Universidade Estadual de Campinas, Brasil, Julho 2010. v, vii, xiii, xv, 4, 6, 9, 57

[20] G. Reinert and S. Schbath, Compound poisson and poisson process approximations for occurrences of multiple words in markov chains, Journal of Computational Biology 5 (1998), 223-254. xiii

[21] Compound poisson approximations for occurrences of multiple words, Ergodic Theory and Dynamical Systems 30 (2010), 159-179. xiii

[22] E. Roquain and S. Schbath, Improved compound poisson approximation for the number of occurrences of multiple words in a stationary markov chain, Advances in Applied Probability 39 (2007), 1-13. xiii

[23] Y. Rozanov and V. Wolkonski, Some limit theorems for random functions, part 1, Theory Probab. Appl (1959), no. 4, 178-197. 2

[24] Walter Rudin, Real and complex analysis, 3th ed., 1987. 47

[25] B. Saussol, S. Troubetzkoy, and S. Vaienti, Recurrence, dimensions and lyapunov exponents, Journal of Statistical Physics 106 (2002), 623-634. xiv, 7

[26] K. Sigmund, On dynamical systems with the specification property, Trans. Amer. Math. Soc 190 (1974), 285-299. 7 


\section{Índice Remissivo}

Alfabeto, 1

Coeficiente $\alpha$-mixing , 2

Coeficiente $\beta$-mixing , 1

Conjunto $B_{n}, 5$

Conjunto $R_{n}, 6$

Dependência tipo $\alpha$-mixing , 2

Dependência tipo $\beta$-mixing , 1

Função $S_{n}, 4$

Palavra, 1

Primeiro Tempo de Retorno, $T_{n}, 3$

Processo $\alpha$-mixing , 2

Processo $\beta$-mixing , 2

Processo com gramática completa , 2

Tempo de Ocorrência , 3

Tempo de retorno em sistemas dinâmicos , 3 